University of Tennessee Health Science Center

UTHSC Digital Commons

$12-2019$

\title{
A Mixed Methods Evaluation of Patient and Provider Perspectives of Chronic Illness Management Following Kidney Transplantation
}

Catherine Kelley Pantik

University of Tennessee Health Science Center

Follow this and additional works at: https://dc.uthsc.edu/dissertations

Part of the Nursing Commons

\section{Recommended Citation}

Pantik, Catherine Kelley (https://orcid.org/0000-0003-2002-9006), "A Mixed Methods Evaluation of Patient and Provider Perspectives of Chronic Illness Management Following Kidney Transplantation" (2019). Theses and Dissertations (ETD). Paper 500. http://dx.doi.org/10.21007/etd.cghs.2019.0485.

This Dissertation is brought to you for free and open access by the College of Graduate Health Sciences at UTHSC Digital Commons. It has been accepted for inclusion in Theses and Dissertations (ETD) by an authorized administrator of UTHSC Digital Commons. For more information, please contact jwelch30@uthsc.edu. 


\title{
A Mixed Methods Evaluation of Patient and Provider Perspectives of Chronic Illness Management Following Kidney Transplantation
}

\begin{abstract}
Introduction: Inconsistent, fragmented care coordination in kidney transplant recipients (KTRs)-whose management requires long-term, complex care, and multiple handoffs among providers-has been shown to result in suboptimal care and higher costs. In order to move forward in improving long-term outcomes, it is necessary to fully assess current practice patterns with appropriate measures. With a full and accurate picture of how elements of the management plan influence both KTR and the health care provider (HCP), it will be possible to implement changes that improve long-term outcomes.Methods: The Chronic Care Model (CCM) was the framework for the study. A mixed method research approach was employed, integrating quantitative and qualitative methodologies in a single cross-sectional, correlational study with data collected from both KTRs and physicians. The 659 KTRs were selected from a list of KTRs who had received a kidney transplant at Methodist University Transplant Institute (MUTI). Physicians were recruited from a list of 96 referring nephrologists who practice in the region. The quantitative data were dichotomized results from Patient Assessment of Chronic Illness Care (PACIC) and Assessment of Chronic Illness Care (ACIC) questionnaires. Continuous data characteristics of the KTRs and HCPs were summarized, with means and standard deviations and medians and quartiles. Categorical data were reported as proportions. Chi-Square and Fisher's Exact tests, as appropriate, were used to determine if any significant associations existed between categorical independent variables and the scale scores. Continuous variables were analyzed using t-tests and Wilcoxon Rank Sum, as appropriate.For qualitative data, NVivo 10 was used to organize the interviews and focus group discussion. Data were analyzed using five phase thematic content analysis. Results: There was variation in the perceptions of chronic illness management as assessed by the PACIC and the ACIC. The number of hospitalizations, time on dialysis and time with graft were the patient variables most associated with PACIC scores. Type of practice, embedded decision support, time in practice and age were the variables most associated with ACIC scores. Patients and providers recognized coordinated care/ follow- up, education, and community resources as barriers to chronic illness management.Discussion: The initial work presented here sought to clarify patient and provider perceptions of the influence of community resources and policies, as well as healthcare system organization using the CCM as a framework. An understanding of the perceptions and experiences of patients and providers will provide the foundation for future work that will address ways in which productive patient-provider interactions can be enhanced, thereby improving patient outcomes.
\end{abstract}

\section{Document Type \\ Dissertation}

\section{Degree Name}

Doctor of Philosophy (PhD)

\section{Program}

Nursing Science

Research Advisor

Donna K. Hathaway, Ph.D.

\section{Keywords}

Chronic Care Management, Kidney Transplantation, Mixed Methods 


\section{Subject Categories}

Medicine and Health Sciences | Nursing 


\section{A Mixed Methods Evaluation of Patient and Provider Perspectives of Chronic Illness Management Following Kidney Transplantation}

Author:

Catherine Kelley Pantik
Advisor:

Donna K. Hathaway, Ph.D.

A Dissertation Presented for The Graduate Studies Council of

The University of Tennessee Health Science Center

in Partial Fulfillment of the Requirements for the

Doctor of Philosophy degree from

The University of Tennessee

in

Nursing Science

College of Graduate Health Sciences

December 2019 
Copyright (C) 2019 by Catherine Pantik. All rights reserved. 


\section{DEDICATION}

I dedicate this dissertation to the patients and providers who participated in this study. Their contributions will provide the cornerstone for the development of a new model of care to improve the quality of life and clinical outcomes for kidney transplant recipients and serve to bring fulfillment to those providing care to people with kidney disease.

I also dedicate this dissertation to my family: my husband Martin; my children Will, Lukas, and Tomas; and my parents Bob and Carolyn Kelley. This journey would not have been possible without their unwavering support in all of my endeavors.

"Ultimately, the secret of quality is love. You have to love your patient, you have to love your profession, you have to love your God. If you have love, you can then work backward to monitor and improve the system."

Donabedian 


\section{ACKNOWLEDGEMENTS}

I would like to acknowledge Dr. Donna Hathaway, my dissertation committee chair. Thank you for sharing your wealth of knowledge in research in general and in kidney transplant specifically. Thank you for your patience and guidance in this project, and for the many opportunities I was afforded through your support.

I thank all of the members of my committee: Dr. Mona Wicks, who shared her broad knowledge of nursing theory through numerous conversations about transition theory and conceptual frameworks to guide this study design; Dr. Muammer Cetingok, whose research in quality of life and social determinants of health provided the perfect complement to this exploratory study; Dr. Elizabeth Tolley, for providing guidance in this study, and whose instruction in biostatistics and multiple regression almost convinced me that I wanted to pursue a degree in statistics; and Dr. Carolyn Graff, whose enthusiasm for qualitative and mixed methods research is contagious and inspirational. I would like to thank each of you for your patience and continued encouragement throughout the years.

I would also like to acknowledge Curtis Roby whose assistance with the electronic survey design, manuscript formatting, tables, figures, and graphs was invaluable.

Finally, I would like to specifically recognize my father, Dr. Bob Kelley, whose care for people with kidney disease spans more than a half-century in this community. His dedication to and love for his patients inspires me daily. 


\begin{abstract}
Introduction: Inconsistent, fragmented care coordination in kidney transplant recipients (KTRs) - whose management requires long-term, complex care and multiple handoffs among providers - has been shown to result in suboptimal care and higher costs. In order to move forward in improving long-term outcomes, it is necessary to fully assess current practice patterns with appropriate measures. With a full and accurate picture of how elements of the management plan influence both KTR and the health care provider (HCP), it will be possible to implement changes that improve long-term outcomes.
\end{abstract}

Methods: The Chronic Care Model (CCM) was the framework for the study. A mixed method research approach was employed, integrating quantitative and qualitative methodologies in a single cross-sectional, correlational study with data collected from both KTRs and physicians. The 659 KTRs were selected from a list of KTRs who had received a kidney transplant at Methodist University Transplant Institute (MUTI). Physicians were recruited from a list of 96 referring nephrologists who practice in the region. The quantitative data were dichotomized results from Patient Assessment of Chronic Illness Care (PACIC) and Assessment of Chronic Illness Care (ACIC) questionnaires. Continuous data characteristics of the KTRs and HCPs were summarized, with means and standard deviations and medians and quartiles. Categorical data were reported as proportions. Chi-Square and Fisher's Exact tests, as appropriate, were used to determine if any significant associations existed between categorical independent variables and the scale scores. Continuous variables were analyzed using t-tests and Wilcoxon Rank Sum, as appropriate.

For qualitative data, NVivo 10 was used to organize the interviews and focus group discussion. Data were analyzed using five phase thematic content analysis.

Results: There was variation in the perceptions of chronic illness management as assessed by the PACIC and the ACIC. The number of hospitalizations, time on dialysis, and time with graft were the patient variables most associated with PACIC scores. Type of practice, embedded decision support, time in practice, and age were the variables most associated with ACIC scores. Patients and providers recognized coordinated care/followup, education, and community resources as barriers to chronic illness management.

Discussion: The initial work presented here sought to clarify patient and provider perceptions of the influence of community resources and policies, as well as healthcare system organization, using the CCM as a framework. An understanding of the perceptions and experiences of patients and providers will provide the foundation for future work that will address ways in which productive patient-provider interactions can be enhanced, thereby improving patient outcomes. 


\section{TABLE OF CONTENTS}

CHAPTER 1. INTRODUCTION .......................................................................................1

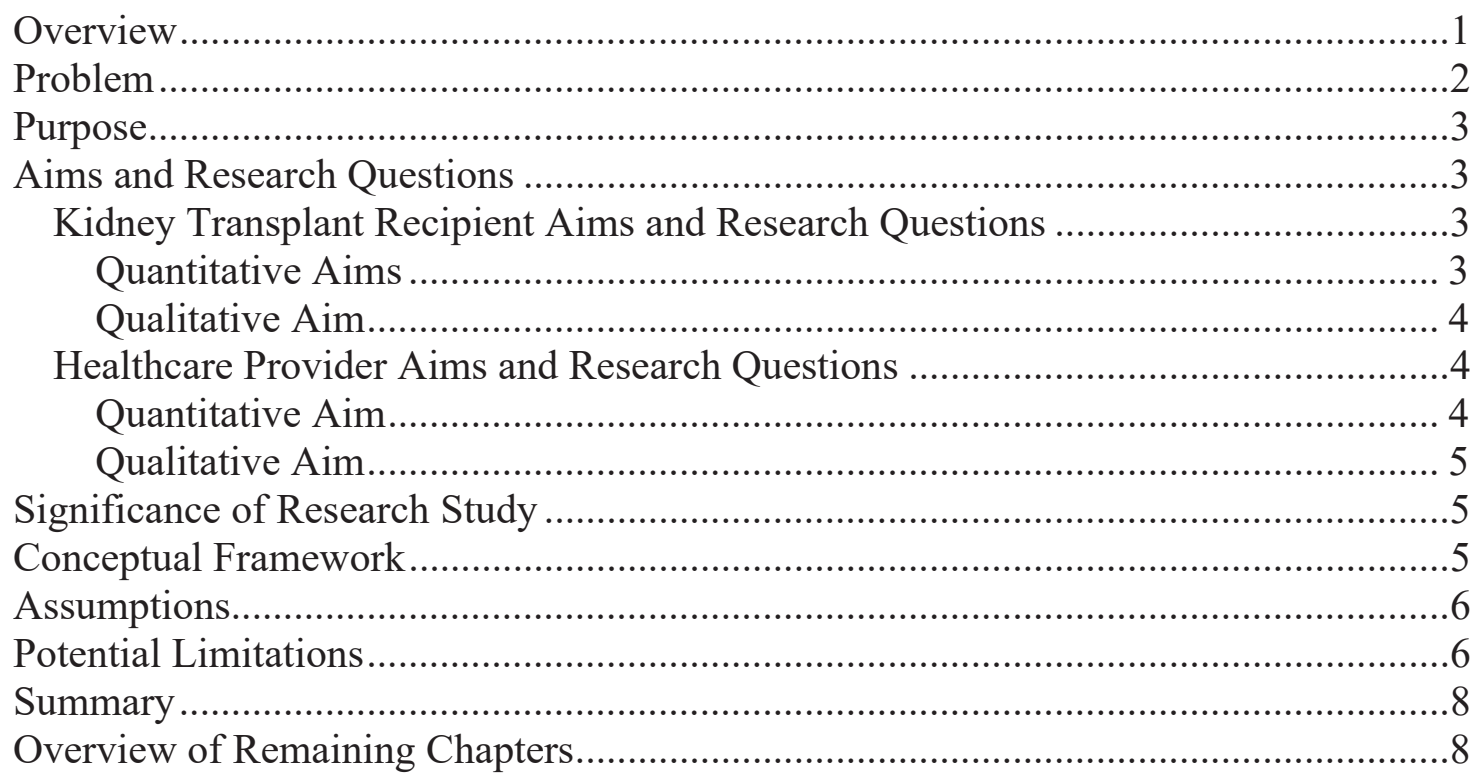

CHAPTER 2. EXPLORING THE CHRONIC CARE MODEL AS A FRAMEWORK FOR EVALUATING CHRONIC ILLNESS MANAGEMENT OF KIDNEY TRANSPLANT RECIPIENTS \& TRANSPLANT OUTCOMES.........9

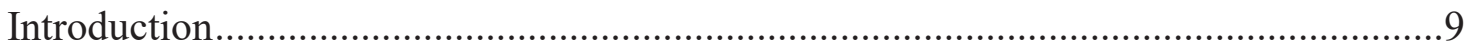

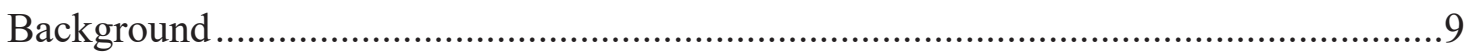

Challenges to Kidney Transplant Chronic Illness Management …………...................10

Recipient Challenges .........................................................................................11

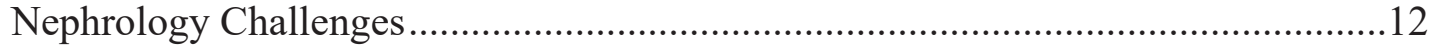

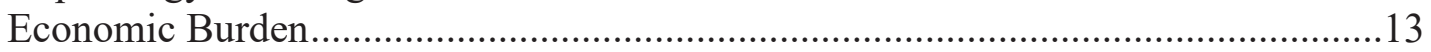

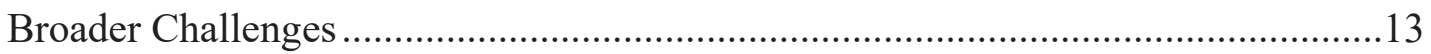

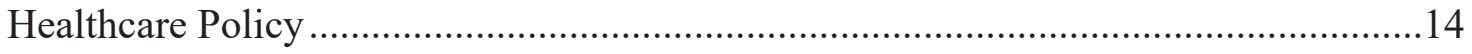

Conceptual Framework for Long-Term KTR Management.......................................14

The Chronic Care Model ...................................................................................14

Community Resources and Policies.......................................................................

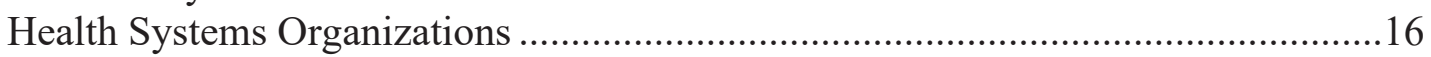

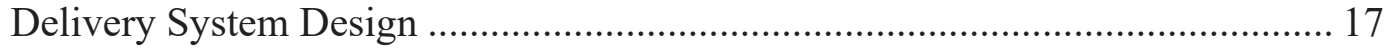

Self-Management Support …………………………................................. 18

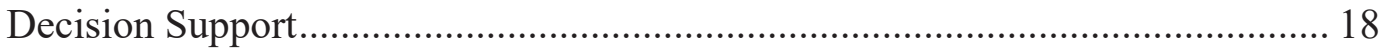

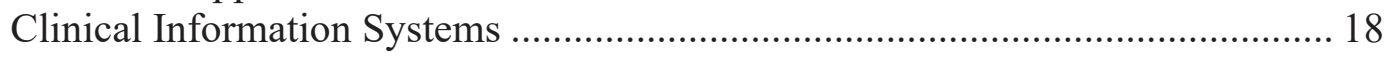

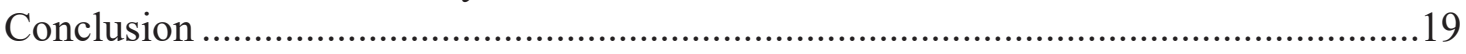

CHAPTER 3. METHODOLOGY AND DESIGN_.......................................................20

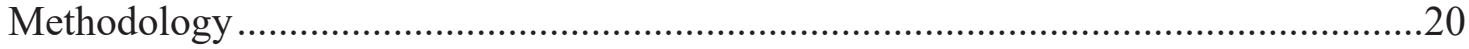

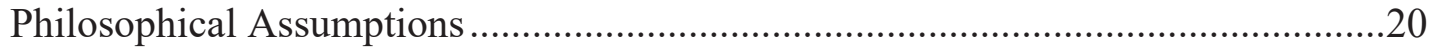

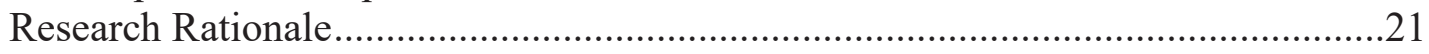




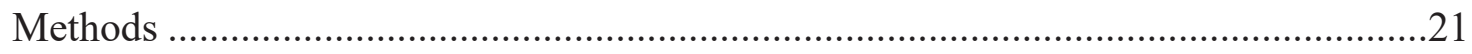

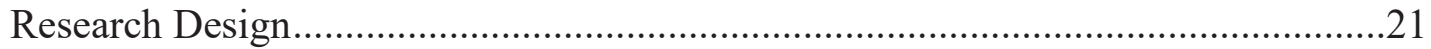

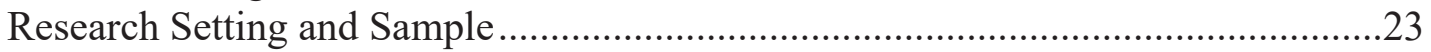

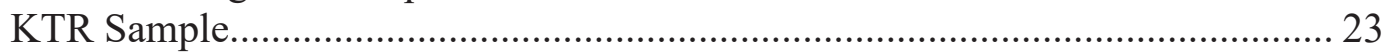

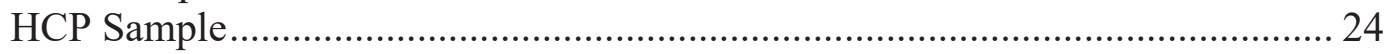

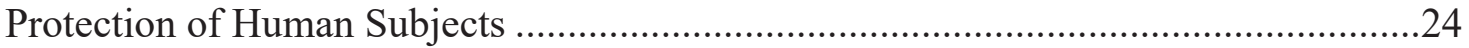

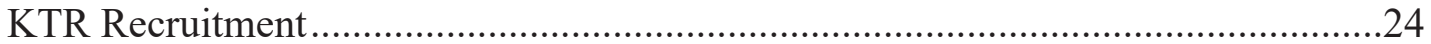

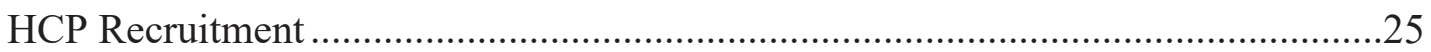

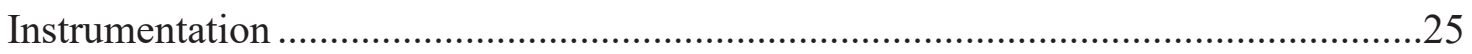

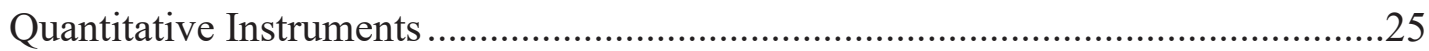

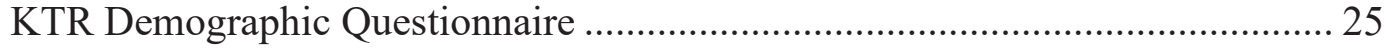

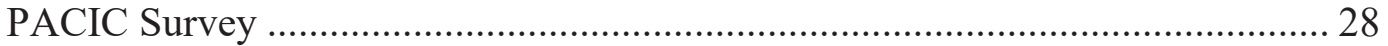

HCP Demographic Questionnaire................................................................ 28

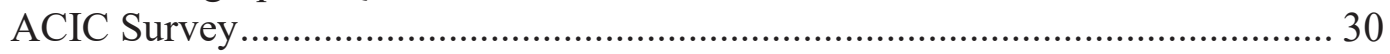

Qualitative Components...................................................................................

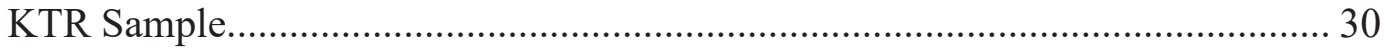

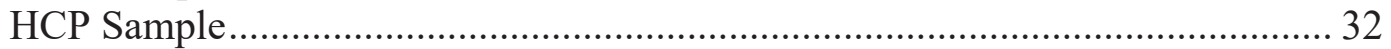

Data Collection, Management, and Analysis..............................................................32

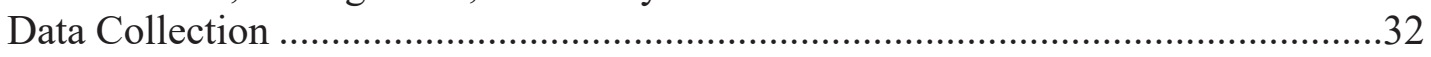

KTR Collection Procedures ............................................................................... 32

HCP Collection Procedures …………........................................................... 33

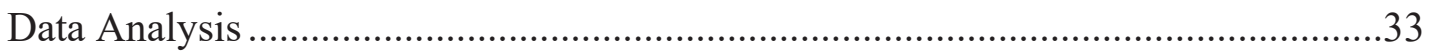

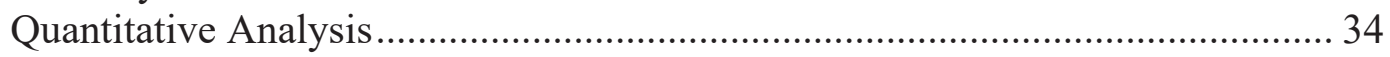

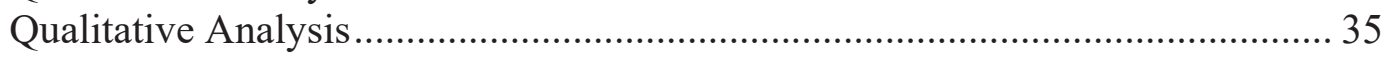

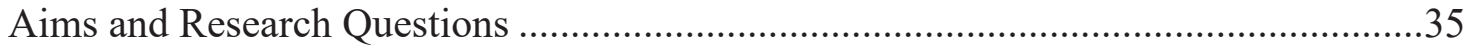

Kidney Transplant Recipient Aims and Research Questions .....................................35

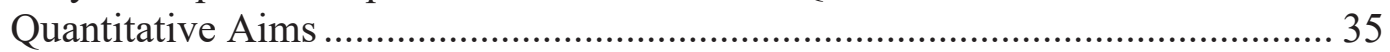

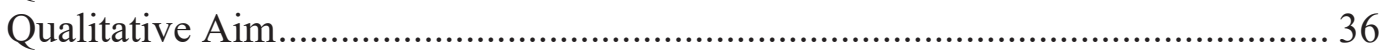

Healthcare Provider Aims and Research Questions ...................................................36

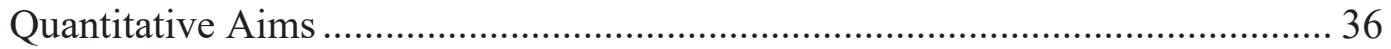

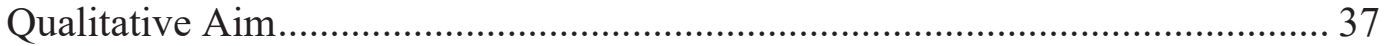

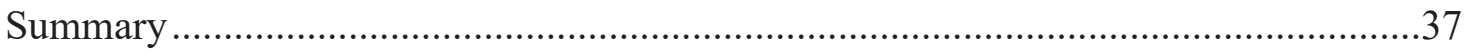

CHAPTER 4. RESULTS.............................................................................................39

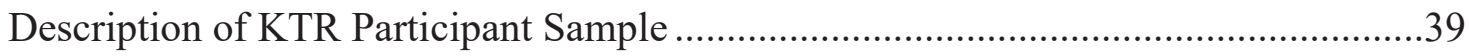

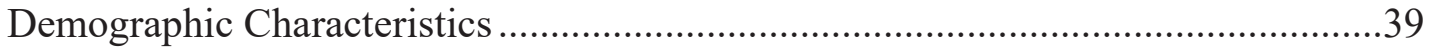

Transplant and Personal Health Management Characteristics....................................39

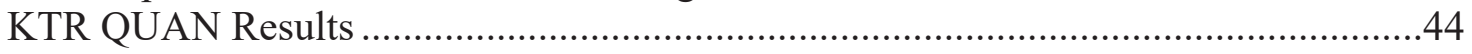

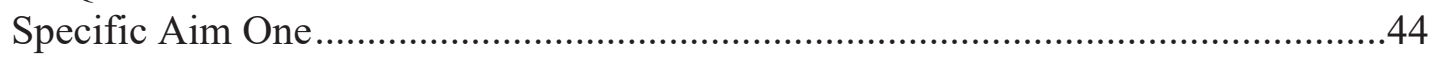

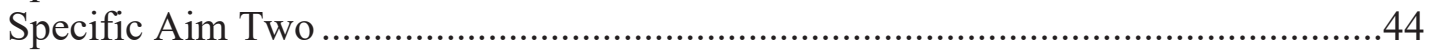

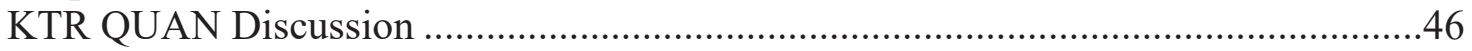

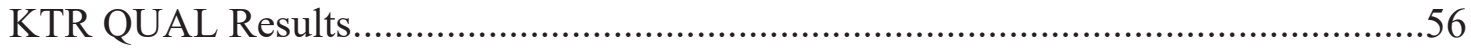

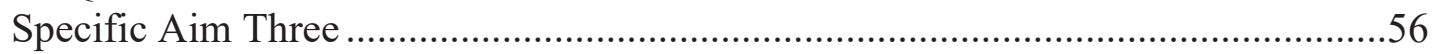

Barriers to Effective Care Management...................................................................57

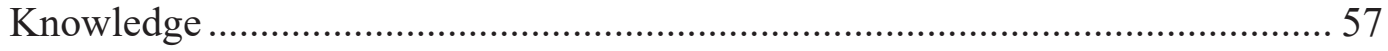

Care Delivery …………………………………….................................. 58 
Community Resources ................................................................................... 59

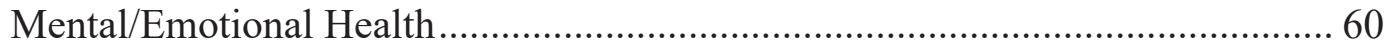

Facilitators to Effective Care Management ………………..................................61

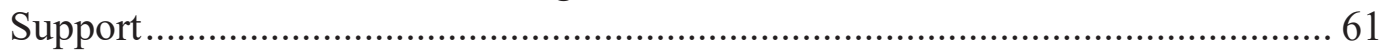

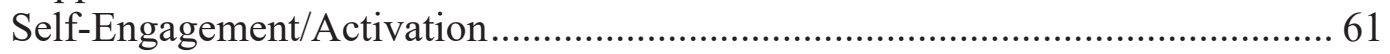

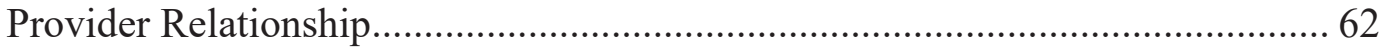

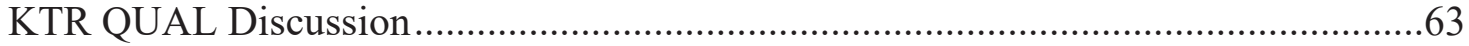

KTR QUAN + QUAL Integration ......................................................................64

Demographic Characteristics of HCP Participant Sample........................................69

Practice Pattern Characteristics of HCP Participant Sample ......................................69

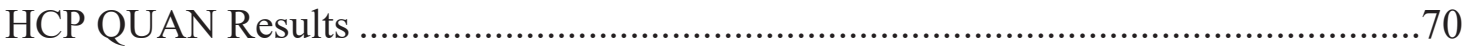

Specific Aim Four .........................................................................................

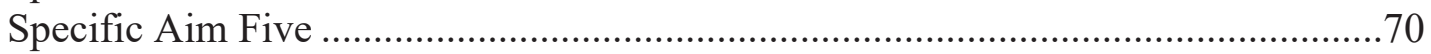

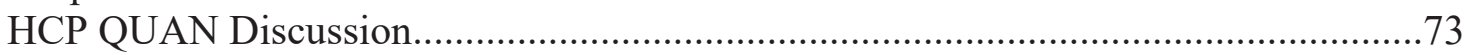

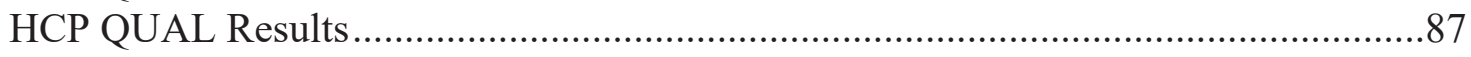

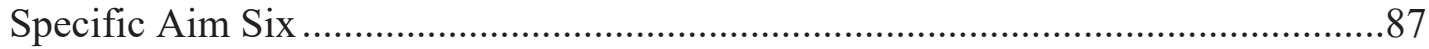

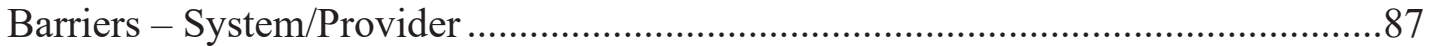

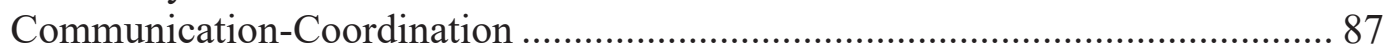

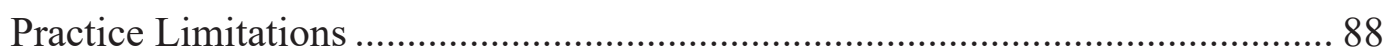

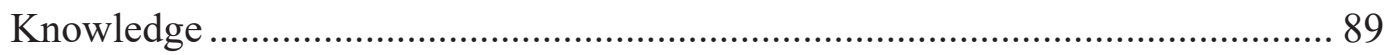

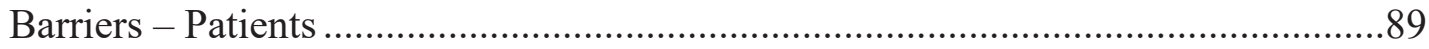

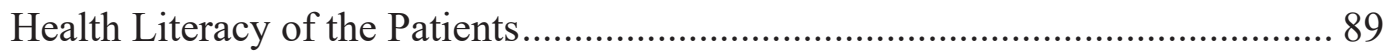

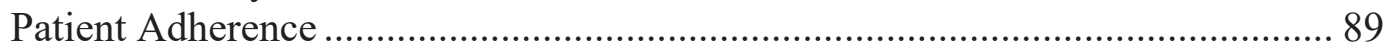

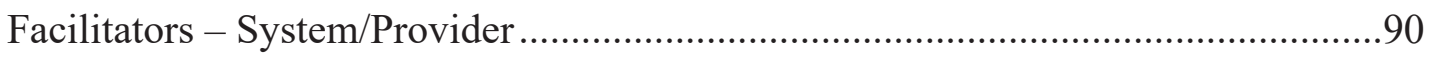

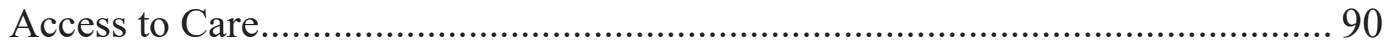

Provider Relationship........................................................................... 90

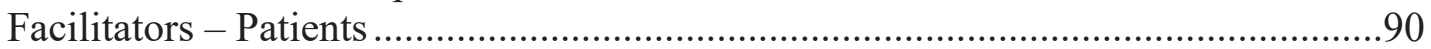

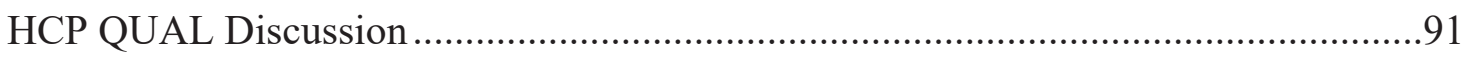

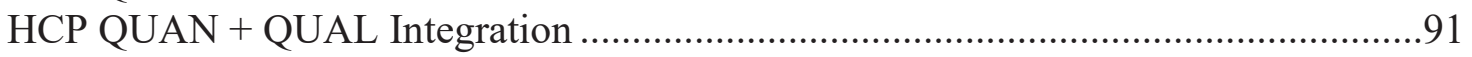

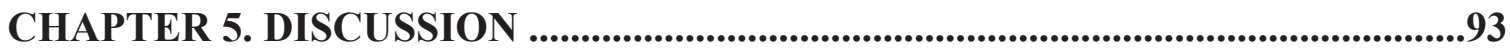

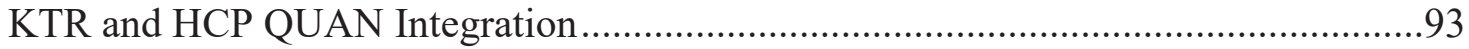

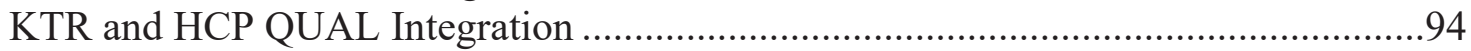

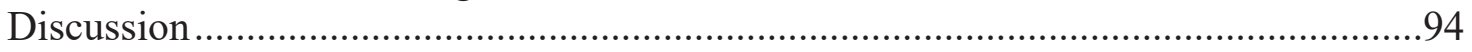

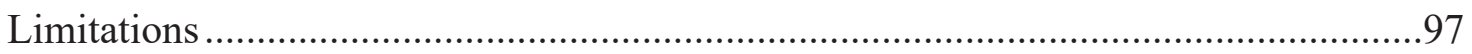

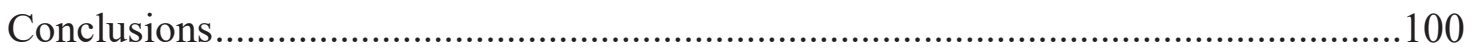

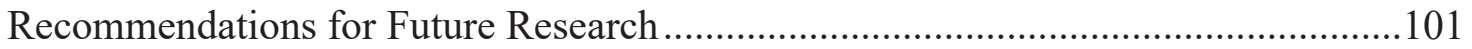

LIST OF REFERENCES ................................................................................................103

APPENDIX A. KTR-INFORMED CONSENT _................................................109

APPENDIX B. KTR DEMOGRAPHIC QUESTIONNAIRE .......................................115

APPENDIX C. PACIC SURVEY ........................................................................................119

APPENDIX D. HCP DEMOGRAPHIC QUESTIONNAIRE ......................................121 
APPENDIX E. ACIC SURVEY

APPENDIX F. KTR FOCUS GROUP GUIDE...............................................................134

APPENDIX G. HCP INTERVIEW GUIDE ................................................................135

APPENDIX H. HCP COVER LETTER.......................................................................136

APPENDIX I. KTR COVER LETTER ...............................................................138

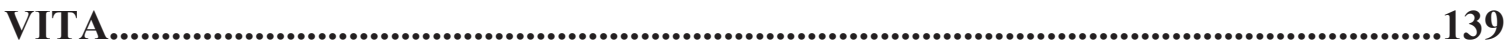




\section{LIST OF TABLES}

Table 3-1. Description of patient assessment of chronic illness management concepts

Table 3-2. Description of chronic illness management concepts

Table 4-1. Descriptive statistics for summaries of kidney transplant recipient continuous variables

Table 4-2. Summaries of kidney transplant recipient demographic characteristics .......41

Table 4-3. Clinical and health characteristics of kidney transplant recipients .....

Table 4-4. Personal health management characteristics of kidney transplant recipients

Table 4-5. PACIC subscales and summary scores $(n=109)$

Table 4-6. Associations between kidney transplant recipient characteristics and the PACIC patient activation subscale

Table 4-7. Associations between kidney transplant recipients' characteristics and the PACIC delivery system design subscale....

Table 4-8. Associations between kidney transplant recipients' demographic characteristics and the PACIC goal-setting subscale

Table 4-9. Associations between kidney transplant recipients' demographic characteristics and the PACIC collaborative problem-solving subscale......50

Table 4-10. Associations between kidney transplant recipients' demographic characteristics and the PACIC follow-up/coordination subscale.....

Table 4-11. Associations between kidney transplant recipients' demographic characteristics and the PACIC total scale score

Table 4-12. Comparison of dichotomized PACIC scores and time spent on dialysis with the perception of high or low implementation of CCM concepts in post-transplant follow-up care.

Table 4-13. Comparison of dichotomized PACIC scores and time with current graft with the perception of high or low implementation of CCM concepts in post-transplant follow-up care.

Table 4-14. Comparison of QUAN PACIC scores and QUAL focus group data..... .66

Table 4-15. Demographic and practice pattern characteristics of physicians $(\mathrm{N}=25) \ldots . .71$ 
Table 4-16. Descriptive statistics for summaries of HCPs continuous variables $(n=25)$

Table 4-17. Assessment of Chronic Illness Care subscale and summary scores $(\mathrm{N}=25)$

Table 4-18. Associations between healthcare provider characteristics and dichotomized Assessment of Chronic Illness Care subscale organization

of healthcare delivery .74

Table 4-19. Associations between healthcare provider characteristics and dichotomized Assessment of Chronic Illness Care subscale community linkages

Table 4-20. Associations between healthcare provider characteristics and dichotomized Assessment of Chronic Illness Care subscale selfmanagement support

Table 4-21. Associations between healthcare provider characteristics and dichotomized Assessment of Chronic Illness Care subscale decision support

Table 4-22. Associations between healthcare provider characteristics and dichotomized Assessment of Chronic Illness Care subscale delivery system design .78

Table 4-23. Associations between healthcare provider characteristics and the dichotomized Assessment of Chronic Illness Care subscale clinical information systems

Table 4-24. Associations between healthcare provider characteristics and the dichotomized Assessment of Chronic Illness Care subscale integration of CCM components .80

Table 4-25. Associations between healthcare provider characteristics and the dichotomized Assessment of Chronic Illness Care summary scores

Table 4-26. Comparison of the dichotomized Assessment of Chronic Illness Care Scores with healthcare provider age and years in practice.....

Table 4-27. ACIC subscale and summary scores $(\mathrm{N}=25) \ldots$

Table 5-1. Comparison of the qualitative results from the focus group and interviews

Table 5-2. Possible system implementation of Chronic Care Model concepts and goals for kidney transplant recipient management. 


\section{LIST OF FIGURES}

Figure 1-1. The Chronic Care Model ..........................................................................

Figure 3-1. Convergent parallel design of the study...............................................22

Figure 3-2. Kidney transplant recruitment flow diagram ........................................26

Figure 3-3. Healthcare provider recruitment flowchart ..........................................27 


\section{LIST OF ABBREVIATIONS}

$\begin{array}{ll}\text { AA } & \text { African American } \\ \text { AD } & \text { Associate's Degree } \\ \text { ACIC } & \text { Assessment of Chronic Illness Care } \\ \text { ACO } & \text { Accountable Care Organization } \\ \text { CCM } & \text { Chronic Care Model } \\ \text { CKD } & \text { Chronic Kidney Disease } \\ \text { CMS } & \text { Centers for Medicare \& Medicaid Services } \\ \text { EMR } & \text { Electronic Medical Record } \\ \text { ESCO } & \text { End-Stage Renal Disease Accountable Care Organization } \\ \text { ESRD } & \text { End-Stage Renal Disease } \\ \text { HCP } & \text { Healthcare Provider } \\ \text { ICIC } & \text { Improving Chronic Illness Care } \\ \text { IOM } & \text { Institutes of Medicine } \\ \text { IQR } & \text { Interquartile Ranges } \\ \text { KTR } & \text { Kidney Transplant Recipient } \\ \text { MUTI } & \text { Methodist University Transplant Institute } \\ \text { NQF } & \text { National Quality Forum } \\ \text { PACIC } & \text { Patient Assessment of Chronic Illness Care } \\ \text { PMP } & \text { Per Million People }\end{array}$




\section{CHAPTER 1. INTRODUCTION}

\section{Overview}

Nearly two decades have passed since the publication of the seminal reports, To Err is Human (Committee on Quality Healthcare in America, Institute of Medicine, 2001) and Crossing the Quality Chasm: A New Health System for the 21st Century (Kohn, Corrigan, \& Donaldson, 2000), which outlined the impact of the fragmented, episodic United States (U.S.) healthcare delivery system on quality and safety of patient care. Since the publishing of these reports, leaders have dedicated considerable resources and research from a variety of disciplines on quality improvement and cost containment across the U.S. healthcare system (Brennan, Mawson, \& Brownsell, 2009). The realization that silos of specialty care in the U.S. are not effective at managing varying degrees of health in an aging population, much of which contends with multiple chronic health conditions, has resulted in an overhaul of healthcare delivery practices and reimbursement strategies (Chung \& Shauver, 2009).

Regardless of the efforts expended over the last two decades, failure to provide healthcare that is consistent with recommended practice guidelines continues to present a significant problem for individuals and society nationwide. Providers' individual practice patterns - defined as their "system" of care (Flocke \& Litaker, 2007) — vary greatly between and among regions of the country. A more comprehensive understanding these systems is an important step in designing interventions that will enhance the delivery of quality, evidence-based care.

Inconsistency in care coordination of chronic illness management—or fragmented care, as it is often called - results in suboptimal care and higher costs (Frandsen \& Joynt, 2015). This is particularly true in the management of patients such as kidney transplant recipients (KTRs) who require long-term complex care as they transition through different stages of posttransplantation care and multiple providers (Gill, Wright, Delmonico, \& Newell, 2017). The transition of care from the transplant clinic to community healthcare providers (HCPs) in particular provides recipients with multiple care plans and medication prescriptions, thereby increasing their vulnerability to poor transition outcomes such as medical errors and nonadherence to vital post-transplant care requirements. Additionally, it is critical to understand the unique health and socioeconomic realities faced by KTRs as the U.S. healthcare system shifts from a volume-based, fee-for-service paradigm to value-based reimbursement models such as the Quality Payment Program of the Medicare Access and CHIP Reauthorization Act (CMS-5517FC, 114th Cong., 2016).

The current state of healthcare presents a clear opportunity for targeted improvement in chronic care management of KTRs. As renowned transplant nephrologist Dr. Meier-Kriesche acknowledged in a presentation at the 2018 Food and Drug Administration's annual workshop, the current clinical endpoint that focuses on 1-year graft failure is of little value (Fowler, 2018). Due to pharmacological advancements in immunosuppressive therapy, 1-year graft failure rates are now approximately 3\% (United States Renal Data System [USRDS], 2018). And although KTRs now live longer lives, the complexity of care required by individuals with renal failure, the high fiscal and moral cost of transplantation, and the subsequent maintenance of graft function 
all provide an excellent platform to examine the questions surrounding the effectiveness of current practice patterns in chronic care management.

Despite scholarly recognition of the importance of chronic illness management to population health, there is currently a lack of widely accepted instruments for the evaluation of chronic illness management (Centers for Medicare and Medicaid Services, [CMS], 2017; Suter et al., 2017). There also is a need to consider clinical- and patient-related outcomes, such as the influence of patient engagement and healthcare delivery system design of long-term care management (Donabedian, 1988; Wagner, 1998). This is especially true for conditions that require complex health interventions, in part because of the variety of concepts employed to describe chronic illness management and programs with varying components implemented to improve the quality of care. Additionally, the National Quality Forum's (NQF) 2013 and 2014 reports to U.S. Congress identified gaps in system-level measures such as integrated delivery systems; shared accountability for health outcomes such as patient and community engagement and fragmented care; measures of clinical quality and outcomes; the importance of the roles of healthcare workers in coordinating care and providing links among the health system, community services, and patients; and patient-centered care and patient-reported outcomes.

Researchers have used Wagner's (1998) Chronic Care Model (CCM) to successfully guide implementation of evidence-based, patient-centered care for chronic illness management; this model has achieved widespread acceptance in practice (Noel, Jones, \& Parchman, 2016; Sendall, McCosker, Crossley, \& Bonner, 2017). Additionally, scholars have used the CCM as a framework to develop two instruments that assess patient and provider experiences in providing and receiving effective chronic illness management, the Patient Assessment of Chronic Illness Care (PACIC) and the Assessment of Chronic Illness Care (ACIC) respectively. The researcher will discuss this model and its instruments in greater detail in subsequent sections.

\section{Problem}

Diabetes, heart disease, and obesity are the most prevalent, preventable, and costly chronic conditions in the United States. Moreover, statisticians evaluating the Mid-South region have consistently reported high burden of these chronic conditions which are major contributors to kidney disease (Jackson et al., 2017). In fact, a 2012 Shelby County community needs health assessment ranked obesity, diabetes, and high blood pressure as the leading issues facing its residents. This region has also been noted for having higher rates of chronic diseases and worse health behaviors than the rest of the U.S. population (Kramer, Black, Matthews, \& James, 2017). These facts further highlight the importance of understanding the chronic care management patterns in this geography, as well as assessing the implementation of the elements of the CCM, which focuses on the influence of systems, policy, patient engagement, and community resources on quality chronic illness management.

The complexity of care, as well as the financial and social factors associated with renal transplants, makes the population of patients extremely vulnerable to poor transition outcomes. To significantly improve the long-term outcomes of these patients, it is necessary to fully assess current practice patterns and patient engagement in their healthcare with appropriate measures. 
By providing policymakers, payers, and community resource agencies with a clear understanding of the complex health and socioeconomic realities faced by transplant recipients and the influence of these factors on care management, it will be possible to ensure that effective and efficient care is available for long-term kidney transplant management.

Although scholars have provided significant evidence in recent years regarding effective models of care for chronic illness, most have excluded KTRs in their investigations. Notably, a multinational study, The CKD Outcomes and Practice Patterns Study specifically excluded KTRs. Furthermore, although significant healthcare resources are dedicated to kidney transplantation, the current researcher could not identify any U.S.-based studies in which the researchers explored the relation of current practice patterns to the characteristics of the healthcare delivery system and the quality of long-term care provided for KTRs.

\section{Purpose}

The current state of healthcare remains characterized by a lack of consistent care coordination as well as a focus on quality measures and patient assessments of care. It is important, therefore, to examine patient and provider perceptions regarding how community resources and policies influence practice patterns and their perceptions regarding the quality of care being provided. Therefore, the purpose of this study was to determine, from the perspectives of the KTR and HCP, the level of chronic illness management in the care of KTRs as they transition to the care of their community providers as well as to describe the barriers and facilitators to care that enhance patient engagement with providers who are prepared with the knowledge and resources to care for KTRs.

\section{Aims and Research Questions}

\section{Kidney Transplant Recipient Aims and Research Questions}

\section{Quantitative Aims}

Specific Aim One. Determine the degree patients believe the long-term care they receive following kidney transplantation aligns with the concepts of quality care as outlined in the CCM.

Research Question 1. How do patients rate their long-term care in the context of concepts identified in the CCM as determined by the PACIC summary and the following subscale scores?

1) patient activation

2) delivery system design/decision support

3) goal-setting

4) collaborative problem-solving

5) follow-up and coordination 
Specific Aim Two. Determine whether demographic and clinical indicators that are associated with kidney graft survival are associated with KTRs' self-reported receipt of longterm care that aligns with the CCM concepts of quality care (PACIC scores).

Research Question 2. Are demographic and clinical characteristics associated with PACIC summary and subscale scores?

\section{Qualitative Aim}

Specific Aim Three. Develop a rich understanding of the transplant experience, including experiences with the larger healthcare system, access to care, coordination of care, relationships with providers, and lifestyle implications.

Research Question 3. How do the KTRs experience the transplant process?

Research Question 4. What barriers and facilitators to quality long-term care are experienced?

\section{Healthcare Provider Aims and Research Questions}

\section{Quantitative Aim}

Specific Aim Four. Determine the degree to which HCPs believe the long-term care provision to their kidney transplant patients aligns with the concepts of quality care as outlined in the CCM.

Research Question 5. How do HCPs rate their overall provision of long-term care in the context of concepts identified in the CCM, as determined by the ACIC summary score and the following subscale scores?

1) organization of the healthcare system

2) community linkages

3) self-management support

4) decision support

5) delivery system design

6) clinical information system

7) integration of the CCM components into the delivery of care

Specific Aim Five. To determine whether demographic and clinical practice patterns are associated with HCPs' self-reported delivery of long-term, follow-up care that aligns with the CCM concepts of quality care.

Research Question 6. Are demographic and practice patterns associated with summary and subscale scores on the ACIC in this sample of HCPs? 


\section{Qualitative Aim}

Specific Aim Six. Develop a rich understanding of the HCPs' experiences with provision of long-term chronic illness care for KTRs, including experiences with the larger healthcare system, access to care, coordination of care, relationships with patients, and personal practice implications.

Research Question 7. How do HCPs experience the care of transplant recipients?

Research Question 8. What barriers and facilitators to the provision of quality long-term care are experienced?

\section{Significance of Research Study}

Through this exploratory study, the researcher aimed to enhance the current understanding of the challenges faced by KTRs and their HCPs by describing quantity and quality-based measures of key stakeholders' perceptions of chronic illness care. The researcher intended that this exploration would provide needed insight into the complex social processes and essential elements of the organization of the healthcare delivery system that influence outcomes. Specifically, the researcher anticipated that by explicating the experiences of the patient and the provider, it would be possible to address the problems related to coordination of care in the kidney transplant population as they transition between multiple care settings.

This insight might inform the development of educational programs and provide a foundation for change in policy and practices to improve long-term outcomes and quality of life for KTRs. This will ensure that effective and efficient integrated care is available for the longterm care management of KTRs.

\section{Conceptual Framework}

In their seminal report on improving healthcare quality in the United States, Crossing the Quality Chasm, the Committee on Quality Healthcare in America (2001) suggested that focus should be placed on removing barriers to quality care and patient adherence rather than placing blame. One approach to achieve this is the inclusion of both providers' and recipients' experiences in the developmental stages of an intervention, which would allow a more comprehensive view of barriers and facilitators to a quality transition (Committee on Quality Healthcare in America, 2001).

Recently, government agencies and other funding sources have declared that studies investigating single factors or only a few risk factors are no longer acceptable (NQF, 2014). The NQF further stated that studies should investigate risk factors for safety and quality of care comprehensively, simultaneously incorporating socioeconomic, treatment- related, conditionrelated, and patient-related factors. 
Chronic disease care management requires acknowledgement of the complex interactions that occur and take place among providers, patients, and systems to ensure optimal health is achieved (Wagner, 1998). Further, in order to better treat chronic disease, care delivery must be delineated. The Chronic Care Model (Figure 1-1), developed in the early 1990s, provides a conceptual framework of the multilevel interactions required for satisfactory quality of care (Coleman, Austin, Brach, \& Wagner, 2009). The CCM includes six main elements: (a) the health system, (b) the delivery system design, (c) decision support, (d) clinical information systems, (e) self-management support, and (f) community resources and policies that facilitate productive interactions between the provider team and the patient.

The CCM is widely accepted for guiding and assessing practice implementation of evidence-based, patient-centered care for chronic illness (Coleman et al., 2009). A primary assumption of the CCM is that improvement in chronic care requires an integrative approach that includes the patient and the provider functioning in a healthcare system that supports coordinated, effective care.

Researchers have suggested that providing healthcare in such an environment would result in prepared, proactive practice teams and informed, activated patients, thereby improving the patient-provider relationship and resulting in improved outcomes (Wagner, 1998). Scholars have shown elements of the CCM to effectively inform the management of persons with diabetes, hypertension, and congestive heart failure - three of the most commonly occurring chronic conditions in KTR; however, previous researchers have not assessed the utility of this model in the KTR population. The researcher will further discuss this model in Chapter 2.

\section{Assumptions}

The samples the researcher recruited were representative of the total population of KTRs receiving a single organ transplant from a Mid-South transplant center and nephrologists providing care to these KTRs in the Mid-South. The researcher assumed the participants' survey responses accurately reflected their opinions and they understood each question and answered all interview questions openly and honestly. Further, the researcher's assumption, based on previous studies, was that these instruments would be psychometrically sound in this study population.

\section{Potential Limitations}

The study had a small sample size, which limited the researcher's capacity for statistical analyses. The findings were limited by the cross-sectional nature of the analysis, restriction to a narrow geographical region of the United States, and selection bias inherent in the convenience samples of HCPs utilized for the study.

Furthermore, selection bias may have occurred in the KTR participants, in that presumably only more healthy KTRs agreed to participate as the study design excluded the hospitalized patient participation. 


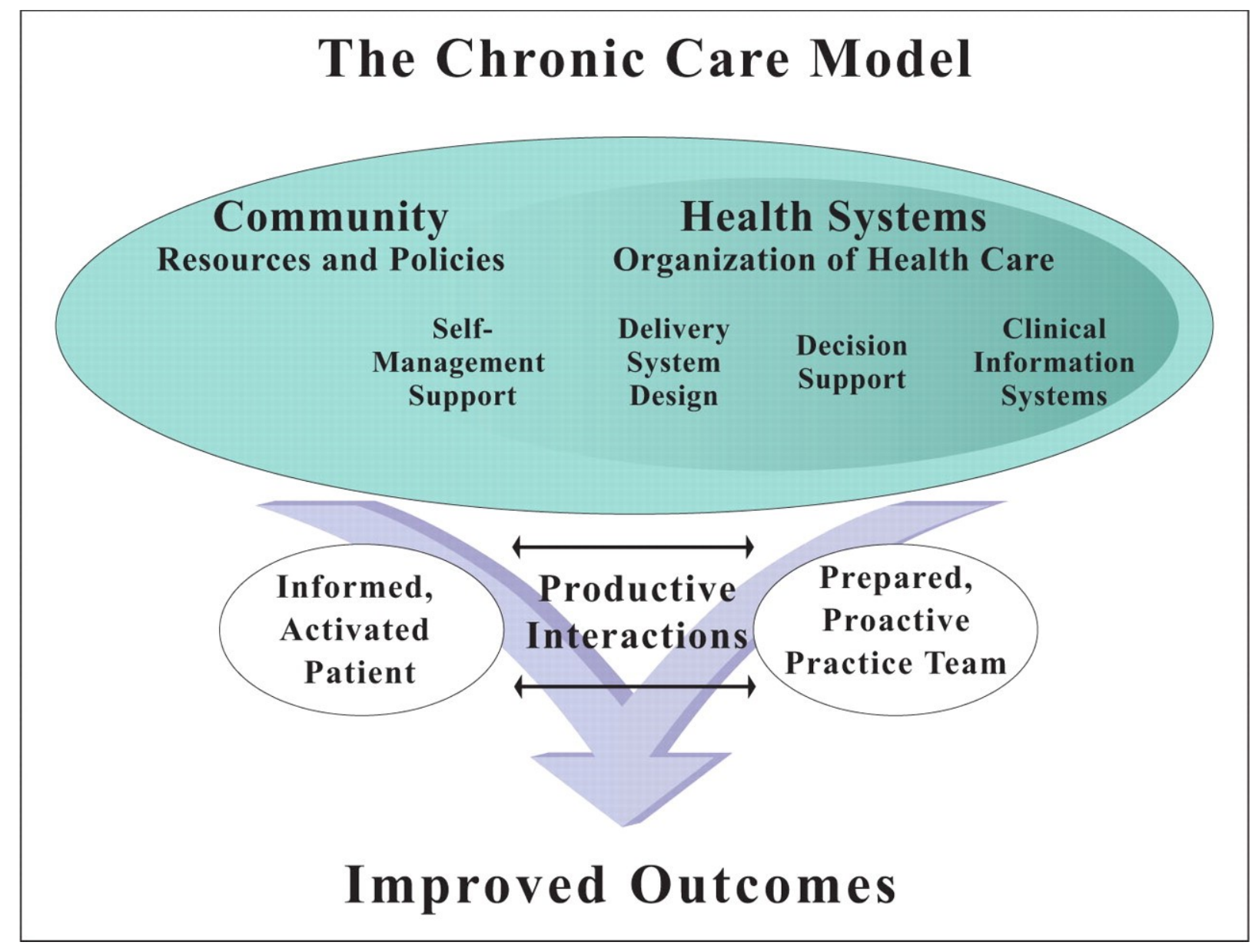

Figure 1-1. The Chronic Care Model

Reprinted with permission. Wagner, E. H. (1998). Chronic disease management: What will it take to improve care for chronic illness? Effective Clinical Practice, 1(1), 2-4. 


\section{Summary}

As a complex subset of persons with chronic illness, kidney transplantation represents a significant portion of healthcare expenditures. Despite the social and fiscal impact, the current researcher could identify no U.S.-based studies that describe the association of practice patterns in KTR long-term management and other factors of healthcare delivery and community resources. The CCM provided a framework for improving clinical outcomes, increasing satisfaction, and reducing costs. Productive interactions between informed patients and prepared providers are at the core of these improved outcomes. Both patients and providers are influenced by various elements of the community and the healthcare system, which may facilitate or impede the management of health and improved outcomes.

Through this study, the researcher aimed to clarify patients' and providers' perceptions of the influence of community resources and policies, as well as the healthcare system organization, using the CCM as a framework. The researcher hoped that an understanding of these individuals' perceptions and experiences would provide the foundation for future researchers to specifically identify ways to enhance productive interactions between these groups, thereby improving patient outcomes.

\section{Overview of Remaining Chapters}

1) In Chapter 1, the researcher introduced the problem to be addressed, the purpose of the study, and the significance of this research. The researcher identified the specific aims of the study, along with a brief description of the conceptual model that provided the framework for the study.

2) Chapter 2 is a conceptual manuscript that includes a critical review of the literature, relevant theories, and a more detailed presentation of the framework supporting the study, with a focus on the long-term care management of KTRs.

3) In Chapter 3, the researcher will present the methodological approach for the study, including the rationale for the mixed methods research design, the sample and setting, the instrumentation and operationalization of variables, and the data analysis procedures.

4) In Chapter 4, the quantitative analysis including descriptive statistics characterizing the KTR participants and their perspectives on the level of implementation of CCM concepts in care management following kidney transplantation will be provided. Following the KTR quantitative results, the qualitative results will be discussed to provide an in-depth description of their experiences and the barriers and facilitators to receiving follow-up care that incorporates the CCM concepts. Finally, the qualitative and quantitative results will be integrated. The HCP results will follow in the same format.

5) In Chapter 5, the KTR and HCP results will be integrated with a discussion of the study findings. Implications for practice, policy, and future research will also be discussed. 


\section{CHAPTER 2. EXPLORING THE CHRONIC CARE MODEL AS A FRAMEWORK FOR EVALUATING CHRONIC ILLNESS MANAGEMENT OF KIDNEY TRANSPLANT RECIPIENTS \& TRANSPLANT OUTCOMES}

\section{Introduction}

Failure to provide coordinated healthcare that is consistent with recommended guidelines presents a significant problem for individuals and society across the United States (Government Accountability Office [GAO], 2016 ). The resulting delivery of care is therefore inconsistent, which compromises the most vulnerable aspects of care. One particularly vulnerable aspect of care occurs during the transition of patient care between settings and providers (Gill et al., 2017).

The current climate of healthcare presents an opportunity for targeted improvement in chronic care management, including that of KTRs who are particularly vulnerable during their transitions of care across multiple specialists and healthcare facilities and ultimately to a primary care provider or nephrologist for ongoing chronic care management (Gill et al., 2017). The complexity of care required by individuals with renal failure, the high social and fiscal costs of transplantation, and the ongoing maintenance of graft function all serve as a platform to examine the questions surrounding the effectiveness of current practice patterns in chronic care management in general as well as in kidney transplant care specifically.

Proposed models for comprehensive kidney care need to go beyond facilitating transplant and must include considerations for long-term management of KTRs. The purpose of this manuscript was to review the literature related to KTRs and chronic care management and to present a conceptual framework to guide redesigning long-term chronic care management for KTRs.

\section{Background}

Advancements in immunosuppressive therapy have resulted in a relatively steady improvement in patient and graft survival for KTRs along with a better quality of life and lower healthcare costs than those experienced by people who remain on dialysis (Königshausen \& Sellin, 2017). These same lifesaving drugs, however, can exacerbate complications and comorbid conditions experienced by KTRs, such as cardiovascular disease and diabetes, both of which are increasingly important because many recipients are older and obese at the time of transplant (Heldal et al., 2010; Khwaja \& El-Nahas, 2012). In addition, cancer, viruses, bone disease, and infection are frequently occurring sequelae (Ong \& Gaston, 2015; Werzowa, Säemann, Haidinger, Krebs, \& Hecking, 2015). Thus, while newer post-transplant regimens are saving more lives and improving quality of life, they also demand the coordination of ongoing care from multiple specialists and ongoing chronic care management from HCPs who may have limited knowledge and experience caring for transplant recipients.

The management of KTRs in the United States can be generally divided into two phases (Vella, 2013): 
1) Early post-operative care, when prevention of acute rejection, optimization of graft function, and prevention of opportunistic infections are the primary goals and

2) Long-term management, which aims to optimize graft function and mitigate long-term consequences of immunosuppression such as malignancies, infections, diabetes, and cardiovascular disease.

Early post-operative care generally extends for the first three months following transplantation. During this time, KTRs are primarily cared for by a transplant specialist and are subsequently transitioned to a community nephrologist, primary care physician, and a host of other specialists for ongoing care (Vella, 2013). This transition to the long-term phase of care, which provides management of ongoing chronic cinditions, generally occurs approximately 3-6 months post-transplant as immunosuppression reaches long-term maintenance levels (Vella, 2013).

\section{Challenges to Kidney Transplant Chronic Illness Management}

Healthcare in the United States has traditionally been provided within an acute care model, whereby care is sought and provided episodically in response to specific problems that the patient is encountering at that time. This approach often involves referral to multiple specialists, making assessment of the quality of care difficult. Recognition that the traditional acute, episodic care model was ineffective in providing quality care for an aging population with multiple chronic conditions spurred changes in reimbursement, tying it to quality measures and patient-reported outcomes (Committee on Healthcare Quality in America, 2001; Squitieri, Bozic, $\&$ Pusic, 2017).

Changes in the reimbursement scheme prompted the development of guidelines from several organizations, which focused on quality and outcomes. For example, the non-profit organization Kidney Disease: Improving Global Outcomes (KDIGO, 2009) provided evidencebased management guidelines supported by the International Society of Nephrology along with several other organizations. These guidelines, however, are not specific as to how care should be delivered. Moreover, long-term care in particular needs to be provided in a manner that involves interventions that are cost-effective and sustainable in addition to promoting improved patient quality of life and outcomes (KDIGO, 2009).

The delivery of care that accomplishes the goals of improved long-term quality of life and outcomes for KTRs while providing cost-effective and sustainable care is challenging in such a complex healthcare environment. In addition to the multiple HCPs and settings where care is delivered, financial and social factors also contribute to poor transitions of care and ultimately patient outcomes (Gill et al., 2017). The transition of care from the transplant clinic to community HCPs often leaves recipients with multiple care plans and medication prescriptions, increasing their vulnerability to medical errors and non-adherence to vital post-transplant care requirements. Although there has been much research dedicated in recent years to effective models of care for chronic illness (Gill et al., 2017), most scholars have failed to specifically address the care of KTRs. Identifying elements of care management that can improve outcomes 
specifically for the growing number of KTRs requires the incorporation of the unique challenges faced by KTRs and the delivery of their care.

\section{Recipient Challenges}

After transplantation, KTRs find themselves needing to learn how to deal with new healthcare and medication regimens, additional chronic health conditions, changing lifestyles, the possibility of losing their new organs, and keeping up with care prescribed by multiple specialists, all while handling new social and economic concerns. At the core of their successful navigation of these new challenges and transitions is their ability to access HCPs with experience caring for KTRs, along with the knowledge of the complex pathophysiological functioning and monitoring that this requires.

Effective early management of these healthcare issues and prevention of complications is imperative for graft and patient survival in addition to overall quality of life (McCaughan \& Courtney, 2015). While researchers have shown transplantation to improve quality of life, HCPs focus more often on maximizing patient and graft survival rather than optimizing outcomes related to comorbidities and quality of life; however, the importance of patient-reported outcomes and quality of life is broadly recognized. The NQF (2014) further states that studies should investigate risk factors for safety and quality of care comprehensively, incorporating socioeconomic, treatment-related, condition-related, and patient-related factors simultaneously.

Despite the perceived improvement in measures of quality of life — such as general health, social function, and work status - there remains a striking difference for KTRs when compared to the general population. For example, work status after kidney transplantation is a specific indicator of recovery that HCPs often leave unaddressed. Researchers have shown that employment status after kidney transplantation has a strong and independent association with patient and graft survival (Tzvetanov et al., 2014). In fact, less than 50\% of KTRs who were employed at the time of transplant return to work during the first-year post-transplant, and these rates decline as time progresses (Tzetanov et al., 2014). This observation further highlights the need for improvement of care during transition that is patient-focused and holistic, considering important social determinants of outcomes in addition to clinical parameters (Tzetanov et al., 2014).

Exacerbating the challenges faced by KTRs is the fact that the majority of patients, prior to transplant, have spent considerable time in the integrated care system of dialysis providers. Moreover, following transplant surgery, KTRs who were Medicare-eligible only due to endstage renal disease (ESRD) must acquire other health insurance by 36 months post-transplant for their ongoing healthcare needs. After the early follow-up period and while navigating the complicated healthcare system, KTRs must now also maneuver through the insurance marketplace. Any lapse in coverage jeopardizes the early identification of worsening kidney function, adequacy of immune suppression therapy, and hypertension and diabetes management, which are crucial to patient and graft survival. 


\section{Nephrology Challenges}

Nephrology practitioners are the major providers of ongoing care for KTRs. Nephrology practitioners are prepared to care for individuals with a broad array of kidney-related conditions, only one of which is transplantation, and the preparation of internists and primary care providers who also provide ongoing care for KTRs is even broader. With the rapid and continuous advances in the field of transplantation, it is of no surprise that these HCPs whose practices generally include only a few KTRs find it difficult to stay current with recent advances in the field of transplantation.

In addition to the challenges of trying to remain current with the most recent advances in complex protocols required for the care of KTRs, the primary care provider also assumes a pivotal role in coordinating care with multiple specialists who are focused on the comorbidities that frequently occur following transplant surgery. Despite the coordination required among multiple HCPs, the complexity of care required, and the importance of long-term management practices for optimal kidney transplantation outcomes, there are no studies that describe kidney transplant management practice patterns. Realizing this lack of information regarding practices in management of KTRs and the variation in outcomes, the American Society of Transplantation (American Society of Transplantation [AST], 2011), with support from Pfizer Medical Education Group, undertook a needs assessment of long-term management of KTRs. Based on surveys and interviews, the AST reported considerable variation in community nephrologists' knowledge, interest, and confidence in managing kidney transplant patients.

Practitioners have also reported that the ongoing care of KTRs presents an additional challenge for the practice site itself. The results of the AST (2011) study indicated that some care providers felt that transplant patients can "fall through the cracks" in busy primary care and nephrology clinics. Many of the practices that provide care for KTRs are small practices, still prevalent in nephrology, which often lack the resources of many larger practices that are necessary to implement the information systems and support staff needed to mitigate many of the coordination of care problems. The additional training, continuous education, and expertise required of individuals necessary to maintain a practice that includes care of KTRs also presents additional financial burdens for practice sites, with personnel expenses growing by $36 \%$ since 2004 and general and administrative expenses growing by 30\% (Huneycutt, 2015).

Even as the worldwide prevalence of kidney disease continues to increase, nephrology faces major workforce challenges as the number of nephrology providers declines (Berns, Ellison, Linas, \& Rosner, 2014). According to the Physician Workforce Data Report from the Association of American Medical Colleges (2015), only 9,000 nephrologists in the United States work more than 20 hours per week in direct patient care. This is coupled with a decline in applicants for fellowship positions from 1.5 applicants per slot in 2010 to 0.8 applicants in 2014 (Salsberg, Quigley, Masselink, \& Xiali, 2015). Sharif, Elsayed, and Stack (2016) suggested that the contributors to the looming shortage are multifactorial, including an aging workforce, a reduced nephrology faculty, cannibalism of nephrology's scope of practice by other specialties, inflexible work schedules, and a need for new care delivery models. Beyond the overall shortage of a nephrology workforce is concern surrounding the distribution of providers. It appears that providers remain in areas close to their training. This is exemplified by the number of 
nephrologists in the District of Columbia, at 63 per million people (PMP), in comparison to those in Iowa, with 13 PMP (Ku, Johansen, Portal, Grimes, \& Hsu, 2015). From these statistics, it is possible to hypothesize that if the discord in supply and demand continues, regional variation in outcomes for KTRs will likewise persist.

\section{Economic Burden}

Today, more than 1 in 4 Americans have multiple chronic conditions, with almost 48 million reporting a disability related to chronic illness (Centers for Disease Control and Prevention [CDC], 2009). The medical care costs associated with chronic illnesses represent $86 \%$ of the $\$ 3$ trillion that the United States spends annually on healthcare (CDC, 2009). As a complex subset of chronic illness expenditures, kidney transplant alone accounts for $\$ 3.1$ billion in Medicare Part A and B expenditures (Organ Procurement and Transplantation Network/Scientific Registry of Transplant Recipients [OPTN/SRTS] Annual Data Report, 2015). This equates to almost $\$ 33,000$ per KTR and does not include expenses covered by Medicare Part D, other payers, or out of pocket.

Although the ESRD population is less than $1 \%$ of the total Medicare population, it accounts for approximately 7\% of Medicare fee-for-service reimbursements based on the most recent report (United States Renal Data Systems [USRDS], 2018).

While costly, successful kidney transplantations yield financial benefits by reducing the number of people who require dialysis, thereby reducing healthcare costs for the government by as much as $\$ 46$ billion per year (Held, McCormick, Ojo, \& Roberts, 2016). According to recent Government Accounting Office (GAO) (2016) analyses, when a transplanted kidney fails, the cost per graft failure is $\$ 88,000$. Moreover, the annual costs of returning to dialysis is approximately $\$ 70,000$ per year.

The financial consequences of kidney failure following transplantation alone warrant serious consideration of how post-transplant care of KTRs could be improved to decrease the incidence of kidney loss.

\section{Broader Challenges}

In addition to the more common acute care model of care delivery and the complexity of care required by KTRs, broader challenges exist related to the KTRs' needs, demands on the practice environment and practitioners, and the financial demands associated with transplantation - which ultimately lie on taxpayers who provide funds for governmental programs that support renal programs. Independently, these challenges represent potentially serious and costly adverse events. Collectively, they increase hospital readmissions that lead to greater healthcare costs (Coleman et al., 2009). The Institute of Medicine has estimated that renewed effort to improve care coordination could result in a $\$ 240$ billion savings in annual healthcare costs (NQF, 2013). 


\section{Healthcare Policy}

Despite the challenges faced by patients, practice sites, and providers, recognizing that the potential benefits of transplantation greatly outweigh the risks has led policymakers and healthcare payers to advocate for increasing access to kidney transplantation (Held et al., 2016). Five objectives were specifically included in Healthy People 2020 to address this goal (Wetmore et al., 2016). In 2014, organizations responsible for organ donor allocation responded with changes in their allocation system to increase the total number of transplanted organs and reduce the number of organs being discarded. Subsequently, patient and graft survival rates slightly decreased, a change that researchers believed to be a function of an increase in transplantation for older adults, more highly sensitized candidates, and those who have been on dialysis for longer periods (U.S. Department of Health and Human Services, 2019). These data highlight the increasing emphasis that needs to be placed on the long-term management practices of KTRs. Thus, care delivery models that provide more efficacious care for chronic conditions associated with KTRs would have the potential to make a major impact on ESRD expenditures, kidney graft survival, and quality of life.

\section{Conceptual Framework for Long-Term KTR Management}

Outcomes research has often focused on one or two elements of healthcare delivery to effect change (Wagner, 1998). However, the practice of compartmentalizing elements of care can be divisive, providing the basis for one party assigning blame to another for poor patient outcomes or increasing costs (Daker-White et al., 2015). Chronic disease care management requires acknowledgement of the complex interactions among providers, patients, and systems to ensure optimal health is achieved (Gill et al., 2017; Wagner, 1998). These critical elements are reflected by the incorporation of the CCM, which was developed in the early 1990s; this model is illustrated previously in Figure 1-1. The CCM incorporates the multilevel interactions required for satisfactory quality of care, which specifically addresses long-term chronic illness management.

\section{The Chronic Care Model}

Wagner (1998) originally developed the CCM as a generic framework to guide the provision of healthcare for primary care patients with a chronic illness in conjunction with the Improving Chronic Illness Care (ICIC) program funded by the Robert Wood Johnson Foundation. This work began with an extensive review of literature to identify strategies used to improve the management of chronic illness. The model was subsequently developed from this review and further improved through surveys of innovative programs and national experts (Coleman et al., 2009).

The CCM has demonstrated its ability to successfully guide implementation of evidencebased, patient-centered care for chronic illness management and has achieved widespread acceptance in practice (Noel et al., 2016; Sendall et al., 2017). A primary assumption of the $\mathrm{CCM}$ is that improvement in chronic care requires an integrative approach that includes the 
patient and the provider functioning in a healthcare system that supports coordinated and effective care as reflected by the constructs enclosed in the large oval in Figure 1-1 (Wagner, 1998). Healthcare that is provided in such an environment results in prepared, proactive practice teams and informed, activated patients, thereby improving the patient/provider relationship by creating productive interactions that result in improved outcomes (Wagner, 1998). Elements of the CCM have been shown to effectively inform the management of persons with diabetes, hypertension, and congestive heart failure - three of the most commonly occurring chronic conditions in KTRs; yet, this model has not been systematically used or evaluated with the KTR population (McCoy et al., 2015; Noel et al., 2016; Sendall et al., 2017). Considering the complex chronic conditions plaguing KTRs, the use of the CCM provides an apt framework to guide improvement of their long-term care management.

The CCM, as depicted in the graphic framework, contains six elements: the healthcare system, community resources, delivery system design, decision support, clinical information systems, and self-management support. These elements influence effective and efficient chronic disease management and facilitate productive interactions between the provider team and patient (Jolly et al., 2015; Tuot et al., 2015). The interaction of these six elements is integral to informed and activated patients cooperating with prepared and proactive practice teams, resulting in improved outcomes. The CCM provides a systematic guide for assessing and restructuring methods of care and outlines and organizes the changes needed in the healthcare system, the practice, and the patient to improve outcomes (Wagner, 1998). Although the model does not offer a quick fix to the current healthcare system, it serves as a multidimensional solution to the very complex problem of chronic disease management (Bodenheimer, Wagner, \& Grumbach, 2002)

The CCM can be applied to a variety of healthcare settings and target populations because it allows the flexibility to explore the local context. Researchers have tested the CCM in settings with different ethnicities and socioeconomic groups (Marcelli et al., 2017; Mahomed \& Asmall, 2017; Wagner, 1998). For instance, Mahomed and Asmall (2017) used the CCM in understanding perceptions and experiences of nurses with the implementation of an integrated CCM in South Africa. Bujang (2017) also used CCM implementation as the focus of a feasibility study involving the primary healthcare system in Malaysia. In the U.S., large healthcare organizations like Kaiser Permanente in California are pioneering CCM implementation, while national healthcare systems drive this development in Europe and Australia.

For KTRs, one of the major obstacles in the implementation of the CCM is smaller nephrology practice size, as the researcher discussed earlier. A recent workforce development study produced for the American Society of Nephrology (2015) reported that more nephrology practices are merging, but the relatively small nephrology practice size remains an impediment to adopting CCM practices. These small practices often lack the resources and personnel necessary to establish an efficacious Delivery System Design, including implementation of systems that would provide Self-Management Support, Decision Support, and Clinical Information Systems. Regardless of the documented successes of the CCM in various practice environments, its use in the KTR population has yet to be documented. In addition, no researchers have performed an assessment of factors contributing to barriers for implementation and to its successful adoption and use. 


\section{Community Resources and Policies}

Partnerships with government agencies, non-profit organizations, and private-sector organizations are vital resources for the healthcare system to support individuals with chronic conditions, particularly those suffering from kidney diseases. Without available community resources, many patients with kidney disease would not have access to specialized care they require and that has been shown to improve health outcomes. Patients' non-health needs — such as housing and social support - are also critical to improving their ability to cope with chronic disease, thus reducing their use of healthcare services; therefore, early identification of KTRs with unmet non-health needs is important.

A partnership with local faith-based organizations, community centers, health services, diabetes education programs, seniors' programs, weight management programs, and/or national patient organizations can supplement the health professional's care for chronically ill patients (Bodenheimer et al., 2002). Community organizations, rather than passively receiving and disseminating health information, can play a vital role in developing culturally relevant models to engage residents in their own health (Headly, 2018). In general, these programs can provide social support, education, and care at a broader community-based level. These community partners provide patients, their families, and their friends with additional opportunities for disease self-management support, educational resources, social support groups, counseling, exercise programs, and smoking cessation programs.

The formalization of referrals and a consultative partnership between the nephrologist and local resources is integral to a collaborative practice (Nugent \& Lambert, 1996; Zwarenstein \& Reeves, 2006). Effective referral to community resources is especially important to older, lowincome, and underserved patients, making this especially important to a large portion of KTRs. Unfortunately, little research exists on this element of the CCM in the context of chronic illness care following a kidney transplant. Community resources are critical elements of the CCM due to their potential to reduce the pressure on the healthcare system and to provide non-health needs that can also contribute to better overall outcomes.

\section{Health Systems Organizations}

Health systems organizations must create a culture that promotes safe and high-quality care. Factors such as financial support from the government, evidence-based guidelines, and formal programs that intend to improve the competence or services of physicians, nephrologists, and chronic kidney disease clinics are necessary healthcare system mechanisms for achieving quality outcomes (Zuccaro, 2015). All of these components of the health systems organization need to be integrated as a single interrelated component that aims to provide quality care to patients. Effective communication is essential for a highly functioning healthcare organization to ensure safe transitions between providers and facilities.

The health systems organization relies on strong leadership for system-wide improvements. Reorganization of a healthcare system requires leadership's commitment to providing resources to implement and sustain best practices in chronic care management. Such a 
reorganization should drive structure changes that lead to process improvements that result in improved outcomes for KTRs in the CCM. Leaders must be committed to system change, but the support staff members are also crucial to successful implementation of change. Leaders must provide clear roles and goals for staff and should provide staff additional time for evaluation and implementation.

\section{Delivery System Design}

Delivery system design is part of the broader framework of health systems organization. A quality delivery system design entails having clinicians who can plan visits in advance and a clear delineation of each provider's and staff member's roles is provided by leadership. The identification of the types of healthcare patient needs and a clarification of roles based on these needs are required in order to reorganize the healthcare delivery system.

Consideration of the processes whereby practices and tools are adopted is a significant aspect of the "delivery system design" concept. For example, coordination of care and collaboration between HCPs are vital components of the delivery of the complex, multidisciplinary care required by KTRs. A healthcare delivery system design with an intentional focus on the way in which care is coordinated would enhance these professional relationships by improving communication and subsequently improving the quality of chronic disease management and patient outcomes (Gordon, Fink, \& Fischer, 2013).

In a study conducted by Rettig, Norris, and Nissenson (2008), nephrologist participants identified poor coordination of care as a major contributor to poor outcomes in chronic kidney disease (CKD) and ESRD. As payers move from the traditional fee-for-service payment model to quality and outcomes payment models, it is necessary for leaders to promote a collaborative practice environment.

Access to care is another major concern in healthcare reform. With the shrinking workforce in nephrology practice, regional disparities in care identified for KTRs, and the return to work as a quality of life indicator for many KTRs, nephrology practice location and clinic hours deserve special attention.

Telenephrology, a term that describes telehealth in terms of its use with nephrology, provides great promise for easing access to care and improving coordination of care (Gordon et al., 2013). Telenephrology has been more widely studied in European healthcare as compared to the United States, with reported success in access to care, coordination, and cost effectiveness (Fernandez, 2006; Prado, Roa, \& Reina-Tosina, 2006; Rumpsfeld, Arild, Norum, \& Breivik, 2005).

Despite the limited number of U.S.-based studies involving telemedicine intervention in nephrology care for CKD, new legislation that provides for Medicare reimbursements is being considered. Several Centers for Medicare and Medicaid Services (CMS) telenephrology reimbursement codes are embedded within the Senate Act 870 (2017) Creating High-Quality Results and Outcomes Necessary to Improve Chronic (CHRONIC) Care Act. The passage of this act will likely lead to more research into successful application of telenephrology. 


\section{Self-Management Support}

Self-management is a critical element in the CCM framework, as the informed and activated patient is a cornerstone to improving health outcomes. The goal of any selfmanagement support program is to empower patients to be active participants in the management of their own health (Lorig \& Holman, 2003). There are several interventions that can be implemented in effective self-management programs, such as the following: tailored education, psychosocial support, goal-setting, identifying barriers to self-care, and effective communication skills.

Despite a dearth of literature regarding self-management support in KTR chronic illness management, researchers have performed investigations related to patient adherence after transplant, which refers to a variety of health behaviors including the taking, timing, and dosing of immunosuppressive medication; taking medication for other co-morbid conditions; attending clinic appointments; self-monitoring for symptoms of infection and rejection; undergoing blood work and other tests in addition to regular exercise, controlling calorie intake, limited - if anyalcohol use, abstaining from tobacco or illicit drug use, and avoiding exposure to the sun (Narva, Norton, \& Boulware, 2015; Steinberg, Moss, Buchanan, \& Goebel, 2017; Varnell et al., 2017).

\section{Decision Support}

A systematic review (Kawamoto, Houlihan, Balas, \& Lobach, 2005) that included 70 randomized control trials of decision support systems found 4 features that were independent predictors of improved clinical practice:

1) support provided automatically as part of clinician workflow

2) support delivered during the time of decision-making

3) provision of specific recommendations

4) computer used to generate the decision support

The authors suggested that these features reflect an ease of access for clinicians, and therefore an effective clinical decision support system must be readily accessible and require minimal clinician effort. Decision support also serves to create more prepared, proactive practice teams and informed, activated patients, as envisioned in the CCM.

\section{Clinical Information Systems}

Clinical information systems allow for tracking of individual patients and specific patient populations (Wagner, Davis, Schaefer, Von Korff, \& Austin, 2002). At the individual level, they provide large volumes of information about needed services and summaries of data related to plans of care. These data from clinical information systems are used to facilitate performance monitoring and quality improvement efforts (Wagner et al., 2002). At the population level, clinical information systems provide data related to groups of patients that are used to help plan all the elements of care identified in the CCM. Similarly, the data derived from the individual 
patient level allows examination of the structure and processes of care in order to identify strategies to improve outcomes.

\section{Conclusion}

The promise of increased funding for kidney disease research from the National Institutes of Health (NIH) and the Department of Health and Human Services, as well as the Kidney Innovation Accelerator (Kidney X) and other research-funding bodies means the time is now to explore innovative models of care and reimbursement plans. In order to move forward in improving long-term outcomes, it is necessary to fully assess current practice patterns with appropriate measures. With a full and accurate picture of how elements of the management plan influence both the KTR and the HCP, it will be possible to implement changes that improve long-term outcomes. Providing policymakers, payers, and community resource agencies with a clear understanding of the complex health and socioeconomic realities faced by transplant recipients and the influence of these factors on care management will also aid in assuring that effective and efficient care is available for long-term kidney transplant management. Concepts within Wagner's (1998) CCM can provide a framework for the evaluation of the long-term management of KTRs as well as the identification and implementation of practice patterns that facilitate high-quality, long-term care following kidney transplantation. 


\section{CHAPTER 3. METHODOLOGY AND DESIGN}

Based on the current state of the science and medical practice, the researcher undertook an evaluation of the long-term management of KTRs and identification of practice patterns that facilitate high-quality, long-term care following kidney transplantation. In this early exploratory study, the researcher used convergent-parallel mixed methods design to obtain quantitative and qualitative data from KTRs and HCPs in order to provide a rich description of the study participants and their perceptions of their current chronic care practices.

In the following sections of this chapter, the researcher will first provide an overview of mixed methods research, the underlying philosophical assumptions for the study, and the rationale for mixed methods use in the research. Next, the researcher will discuss the procedures that the researcher used to obtain the quantitative and qualitative data in detail. Lastly, the researcher will present the methods and measures that the researcher used to analyze both quantitative and qualitative data, along with the methods that the researcher used to integrate these data.

\section{Methodology}

\section{Philosophical Assumptions}

Similar to the singular quantitative and qualitative approaches, mixed methods research is rooted in a specific philosophical foundation. Unlike the paradigm, or worldview, of positivism associated with a quantitative methodology or constructivism of qualitative methodology, pragmatism rejects the "either-or" (Teddlie \& Tashakkori, 2009) and offers a "what works" solution (Creswell \& Plano Clark, 2011) by using both deductive and inductive logic (Teddlie \& Tashakkori, 2009). The pragmatist view is often associated with mixed methodologies (Zhang \& Creswell, 2013). The pragmatist views reality as both singular and multiple; that is, there might be a theory that operates to explain a phenomenon, but it is important to assess varied individual input into the nature of the phenomenon. A pragmatic view is often referred to as multiple ways of knowing.

Whereas quantitative research is focused on measuring and analyzing variables in order to determine their relationships, Denzin and Lincoln (1994) stated that qualitative researchers focus on answering questions that are based on social experiences and the interpretation of individuals. Rooted in the pragmatic philosophical view of mixed methods research, the current researcher utilized multiple data sources to achieve a contextual understanding and recognition of the practice patterns that facilitate high-quality, long-term care following kidney transplantation and the barriers that patients and providers must overcome in order to achieve this goal. 


\section{Research Rationale}

Recent reports have indicated a lack of progress toward accomplishment of the goals set forth by the Committee on Quality Healthcare in America's (2001) Crossing the Quality Chasm: A New Health System for the 21st Century, which aimed to improve the safety and quality of healthcare. Although much research has been conducted in search of ways to accomplish these goals, the multifaceted relationships among environment, health policy, and socioeconomic influences on patient outcomes make the results of such research challenging to interpret.

The use of a mixed methods approach is increasingly sought in healthcare to assess healthcare delivery design, quality of care, and patient and provider perspectives of complex and dynamic care management (Curry et al., 2013). Researchers have provided evidence suggesting that neither quantitative nor qualitative research methods alone can answer highly complex questions such as those presented in healthcare redesign and quality outcomes. The complexity of these questions highlights the need to adopt a more complex methodology such as the mixed methods approach (Morse, 2016) which involves the strategic integration of quantitative and qualitative methodologies in a single study (Zhang \& Creswell, 2013). By combining quantitative and qualitative methods, study findings will be more robust as a result of complementarity, whereby the results from one method enhance the results of the other method (Zhang \& Creswell, 2013). The framework for the current study argues for the importance of collaboration between KTRs and HCPs and an integrated process of care between all providers and the KTR. This collaboration allows for a deep and broad analysis of the research problem. The current researcher sought to incorporate both methods of inquiry to maximize the strengths of the quantitative and qualitative results; this synthesis allowed for the researcher to generate more nuanced and comprehensive results.

\section{Methods}

\section{Research Design}

A convergent parallel mixed methods design was used in the current cross-sectional, exploratory study of chronic illness management in the follow-up care of KTRs. The goal of the convergent parallel design is to obtain different but complementary data with the narrative and numerical data collected within the same time frame and equal emphasis given to both methods (Creswell \& Plano Clark, 2011; Teddie \& Tashakkori, 2009). Equal emphasis on quantitative and qualitative data each collected simultaneously is symbolized by Quan + Qual (Creswell \& Plano Clark, 2011; Teddie \& Tashakkori, 2009). This design was originally referred to as triangulation, as it allows a more complete understanding of the research phenomenon through understanding how the analyses of the two data sets converge or diverge and develop internally confirmed conclusions about a single phenomenon (Creswell \& Plano Clark, 2011). The current study consisted of two convergent parallel studies: one with the patient population and the other with the provider population. Both the quantitative and qualitative strands provided information designed to address interlinked questions. Figure 3-1 provides a diagrammatic representation of the convergent parallel design of the study. 

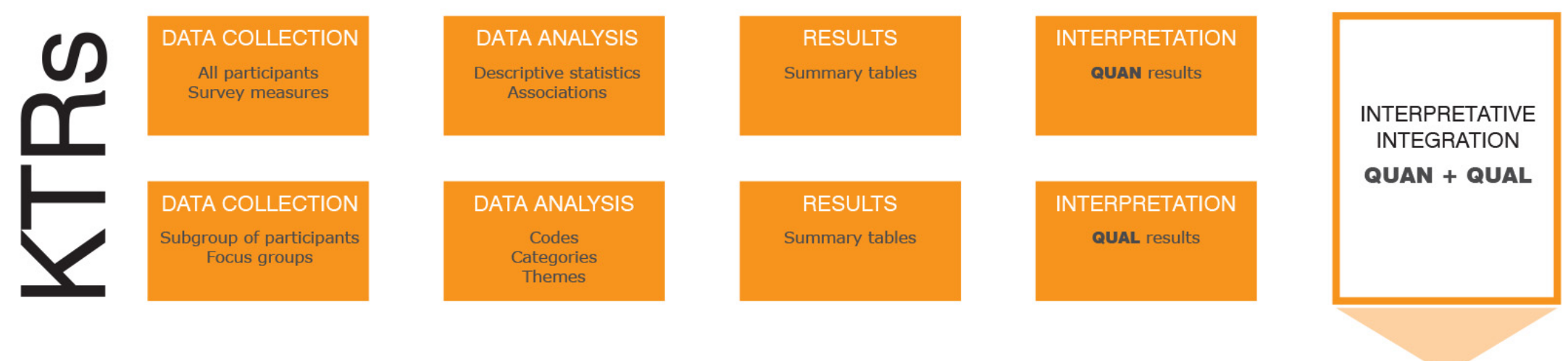

INTERPRETATIVE

MERGED RESULTS

KTRs + HCPs
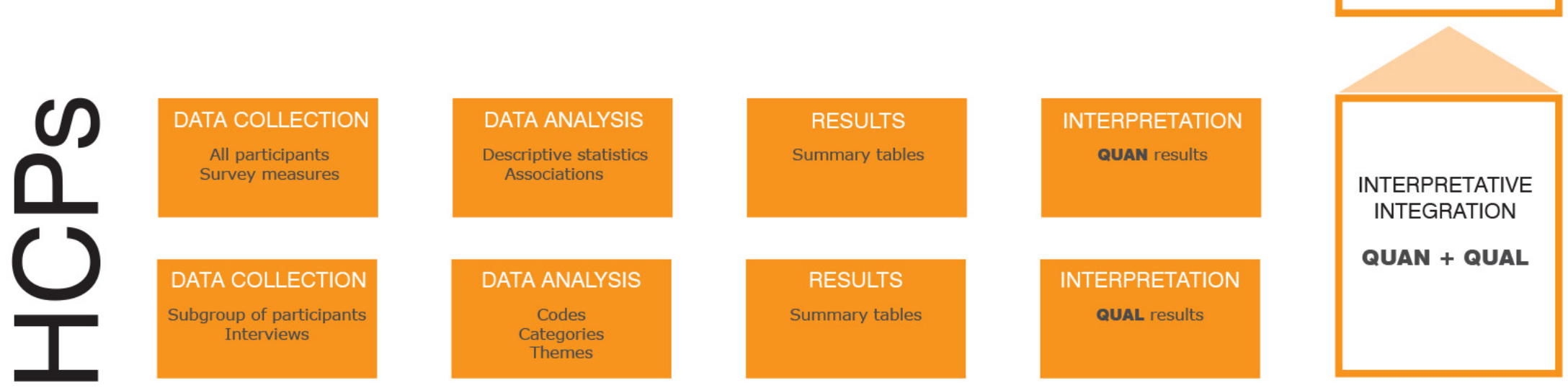

Figure 3-1. Convergent parallel design of the study 


\section{Research Setting and Sample}

Methodist University Transplant Institute (MUTI), part of the Methodist LeBonheur Healthcare System and located in Shelby County, is one of Tennessee's largest healthcare providers. This healthcare system serves populations of diverse socio-economic characteristics across a large area of West Tennessee, North Mississippi, and East Arkansas, collectively known as the Mid-South. Additionally, MUTI has a well-established organ transplant organization established in 1968 known as the University of Tennessee Organ Transplant Program. In 1970, the center performed its first kidney transplant, making MUTI the sixth center to perform a kidney transplant in the United States.

The current researcher recruited two samples through MUTI to provide data and insight regarding the practices and perspectives of long-term management of kidney transplant recipients, KTR, and physician providers, HCP. Neither power analysis nor sample size estimates could be performed because data needed for these computations do not exist for this reference population. Data generated from this project may be used to estimate power for future studies. The researcher, in consultation with the committee member advising the researcher on statistical analysis, determined that a sample size of 50 KTRs and 50 HCPs would be feasible to recruit and should be sufficient to obtain a reasonably wide range of responses and, thereby, estimate the variability of responses from the quantitative instruments used in this study.

\section{KTR Sample}

The researcher randomly recruited the participants for the quantitative strand from a list of people receiving transplants between August 2004 and August 2015 and assumed by MUTI to be living with a functioning graft. The researcher then conveniently selected the KTRs to participate in the qualitative strand from among the same people willing to participate in the quantitative strand. The researcher filtered the list to include people living in West Tennessee, Northern Mississippi, and Eastern Arkansas, all of which are classified as belonging to the MidSouth region, who met the inclusion and exclusion criteria provided below.

KTR Inclusion Criteria. The inclusion criteria for the KTR participants included the following characteristics:

1) Ability to speak and understand English;

2) Being aged 18 years old or older;

3) Having received a kidney transplantation at MUTI between 2004-2015; and

4) Having received long-term, follow-up care from a nephrologist practicing in the MidSouth region for at least 1 year.

KTR Exclusion Criteria. The exclusion criteria for the KTR participants included the following characteristics:

1) Hospitalized at the time of recruitment and

2) Had two different organs transplanted (e.g., kidney/pancreas, kidney/heart). 


\section{HCP Sample}

The researcher recruited a convenience sample of participant HCPs from a list of 96 referring nephrologists provided by MUTI for the quantitative strand. Those HCPs who volunteered to participate in the study were invited to participate in an individual face-to-face interview for the qualitative strand. The inclusion criteria are provided below, and there were no exclusion criteria.

HCP Inclusion Criteria. The inclusion criteria for the HCPs included the following:

1) Practicing as a nephrologist in the Mid-South region, as defined above and

2) Having provided long-term, follow-up care for at least one adult who received a kidney transplant at MUTI

HCP Exclusion Criteria. No exclusion criteria were included for the HCPs in the study.

Kidney recipients were not nested within HCPs for practical reasons. That is, it was unlikely that one nephrologist, or even an entire nephrology practice, could have provided care for sufficient numbers of KTRs for nesting purposes. In addition, it was also unlikely that individual recipients would have seen the same provider during every office visit. Further, various KTRs possibly would have received care from HCPs not included in the study sample.

\section{Protection of Human Subjects}

The University of Tennessee Health Science Center Institutional Review Board (UTHSC/IRB) approved the study objectives, the procedures used in recruitment, and the quantitative and qualitative instruments that the researcher used in data collection. None of the voluntary subjects were classified as vulnerable participants. In this study, survey consent statement was read to participants, and completion of the survey was deemed "granting consent" for the quantitative strand of this study. The researcher obtained KTRs' signed informed consent (Appendix A) prior to the qualitative strand. HCPs' agreement to individual interviews was deemed consent for participation in the qualitative strand.

\section{KTR Recruitment}

The researcher randomly selected the participants from a computer-generated list of all patients meeting study criteria $(n=659)$ and assigned each a random number from 1 to 659 . A random number generator was used to select potential participants for study recruitment. Recruitment of KTR participants commenced in September 2015 and ended in March 2016. The researcher contacted each identified KTR by telephone to confirm eligibility and interest in participation in the study. Contacts with telephone numbers no longer in service were replaced by the next number. Additionally, two attempts were made to contact a potential participant, with messages left when possible, before the next available number on the list was selected. A total of 274 calls were made. The researcher replaced 52 out-of-service telephone contact numbers with 
the next telephone contact number from the random number generator list. The researcher left two telephone messages for $44 \mathrm{KTRs}$ before replacing these potential participants with the next telephone contact number from the random number generator list. Fifty-four KTRs contacted were not interested in participating. Potential KTR participants successfully contacted by phone were asked to re-confirm their eligibility to participate in the study based on inclusion/exclusion criteria. If the participants met the inclusion criteria, the researcher offered the option to participate in the survey completion and focus group. The researcher achieved a recruitment rate of $42 \%$ with the KTR sample. Figure 3-2 provides a flowchart of the KTR recruitment pathway.

\section{HCP Recruitment}

The researcher recruited a convenience sample of physicians from a list of 96 referring nephrologists to MUTI for kidney transplantation. The researcher eliminated nine HCPs due to practice location and determined that three no longer practiced. The researcher included $84 \mathrm{HCPs}$ in the list of potential participants. To maximize recruitment, the researcher used multiple methods including direct contact at the nephrology practice, email, U.S. Postal Service, and a presentation of the research participation opportunity to a group of nephrologists identified on the referral list at an MUTI continuing medical education presentation. HCP recruitment commenced in November 2015 and ended in September 2016. The researcher achieved a recruitment rate of 30\% with the HCP sample. Figure 3-3 provides a flowchart of the HCP recruitment pathway.

\section{Instrumentation}

\section{Quantitative Instruments}

Demographic questionnaires were created (Appendix B) and chronic illness management perceptions assessed with instruments developed by The MacColl Center for Health Care Innovation's Patient Assessment of Chronic Illness Care (PACIC) (Glasgow et al., 2005), which assesses the level of chronic illness management implemented in a healthcare organization from the patient perspective. Since the development of the PACIC (Glasgow et al., 2005), the instrument has been used in over 100 studies to rate the level of patient-reported care congruent with the CCM. Similarly, the Assessment of Chronic Illness Care (ACIC) (Bonomi, Wagner, Glasgow, \& Von Korff, 2002) was designed to assess providers' perspective of providing effective chronic illness management. Likewise, the ACIC has been used in multiple studies to assess healthcare organizations' delivery of care congruent with the CCM. These instruments are described below and are available for download at improvingchroniccare.org.

\section{KTR Demographic Questionnaire}

The investigator-developed KTR Demographic Questionnaire (Appendix B) consisted of 25 continuous, categorical and open-ended questions and served to elicit responses to objective 
MUTI list of KTRs between Aug.

1, 2004 and Aug. 12015 N=727

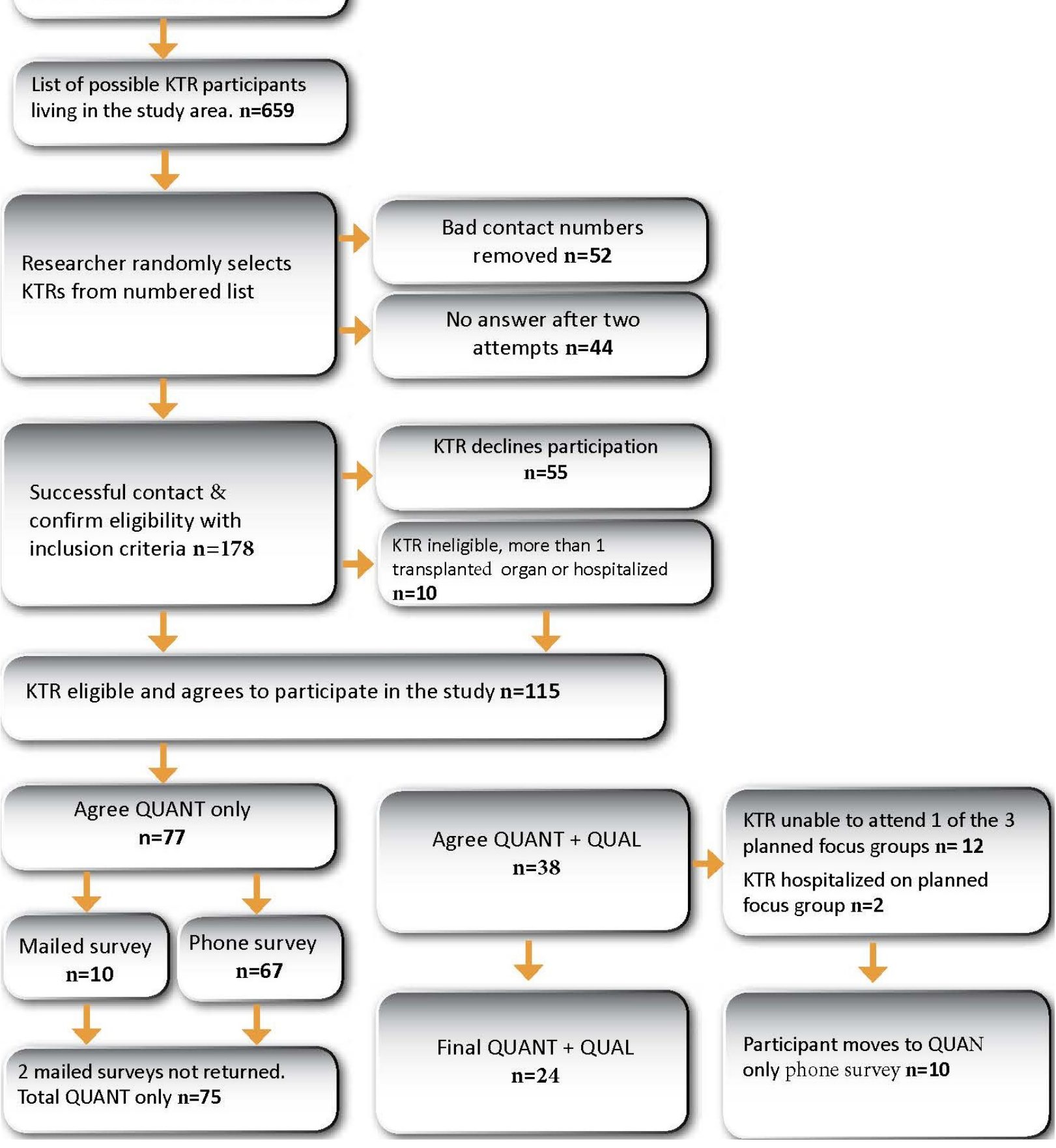

Figure 3-2. Kidney transplant recruitment flow diagram 


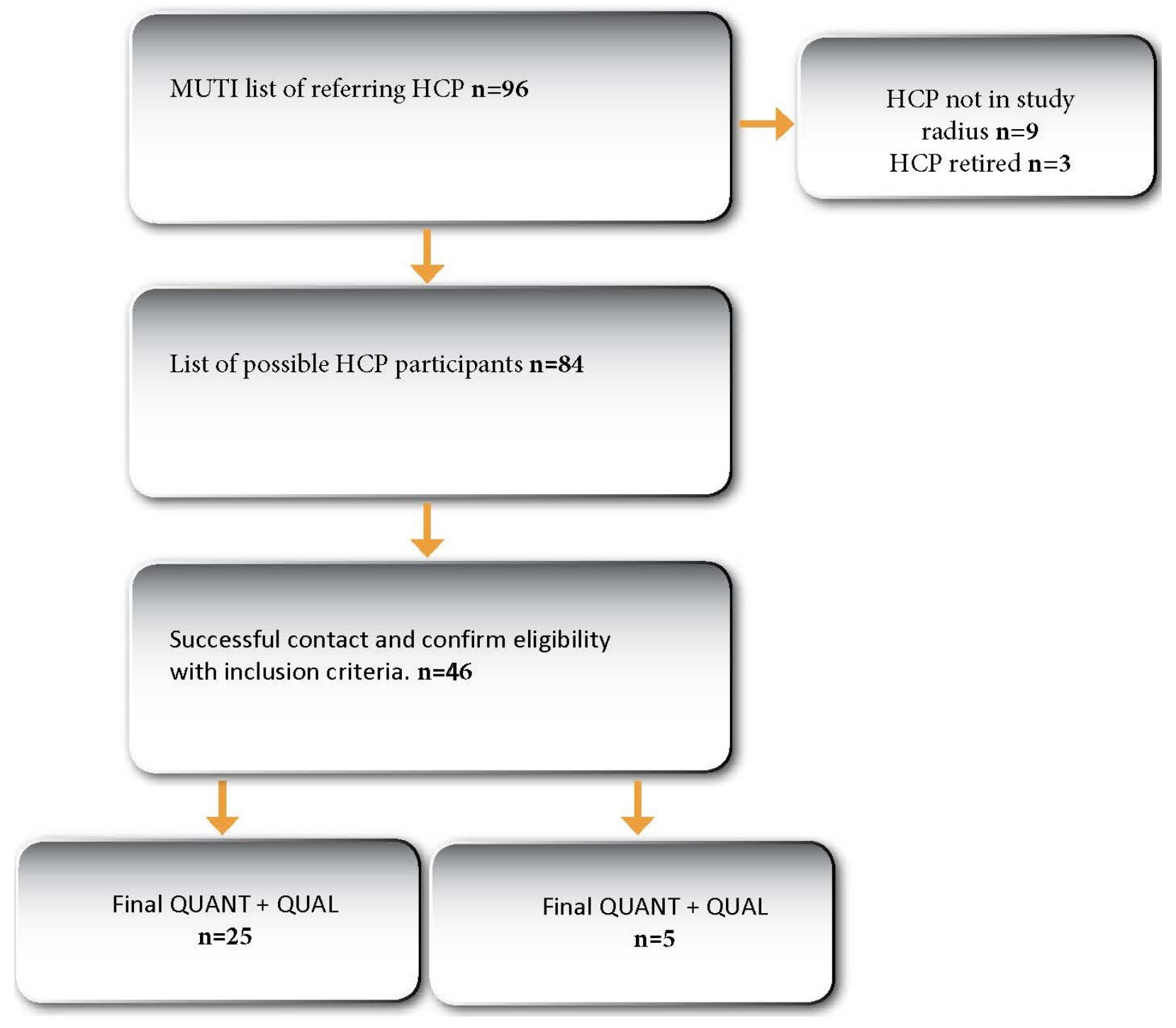

Figure 3-3. Healthcare provider recruitment flowchart 
questions including those regarding socio-demographic status, transplant conditions, self-care, and chronic conditions that are associated with kidney graft survival. Clinical information gathered from chart review included etiology of kidney disease, source of kidney, and immediate or delayed graft function. Patient-reported information included number of kidney transplants, time on dialysis before first transplantation, number of chronic conditions requiring medicalmanagement (other than CKD), and the number of hospitalizations. Personal health management information included smoking history, use of alcohol, and physical activity.

\section{PACIC Survey}

PACIC Survey Measurement Goal. The PACIC Survey (Appendix C) was developed to measure the extent to which patients with chronic illness receive clinical care that is patientcentered, proactive, and planned (Glasgow et al., 2005). The items of this instrument were derived from a pool of 46 items generated by a national panel of experts on chronic illness care and the CCM. A pilot test was done with a separate, earlier sample of 130 patients, and the 20 items were aggregated into five a priori scales based on the key components of the CCM. This 20-item survey consists of five subscales that represent the components of the CCM as experienced by patients: (a) patient activation, (b) delivery system design/decision support, (c) goal-setting, (d) collaborative problem-solving, and (e) follow-up and coordination. Completing the PACIC involved patients rating each item on a 5-point scale in order to indicate how often clinical care or service described was experienced. Scores ranged from 1-5, with higher scores indicating patients' perception that their care is more consistent with the CCM (Rick et al., 2012). A detailed description of the CCM concepts is provided in Table 3-1.

Psychometric Properties. Previous researchers have reported the psychometric properties of the PACIC to be both valid and reliable (Glasgow et al., 2005; Rick et al., 2012). Glasgow et al. (2005) reported that the PACIC was only slightly correlated with age and gender and unrelated to education. Contrary to prediction, it was only slightly correlated $(r=0.13)$ with number of chronic conditions. The PACIC demonstrated moderate test-retest reliability $(r=0.58$ during the course of 3 months) and was correlated moderately, as predicted $(r=0.32-0.60$, median $=0.50, p<0.001)$ to measures of primary care and patient activation. According to Rick et al. (2012), the subscales demonstrated acceptable reliability with internal consistency of alpha reliabilities as follows: patient activation $(0.86,3$ items $)$; delivery system design $(0.68,3$ items); goal-setting (0.82, 5 items); problem-solving (0.86, 4 items); follow-up and coordination (0.82, 5 items); and PACIC total (0.94, 20 items). In terms of the instrument's construct validity, the PACIC has been found valid based on confirmatory factor analysis suggesting that the five subscales measure different aspects of the same broader variable. Researchers have used the PACIC to assess the congruency of care delivery with the CCM concepts of quality care in several chronic diseases and primary care.

\section{HCP Demographic Questionnaire}

The investigator-developed objective questionnaire (Appendix D) for the HCPs consisted of 14 continuous, categorical and open-ended questions used to acquire information about the HCP and clinical practice patterns. These patterns included years in practice, type of 
Table 3-1. Description of patient assessment of chronic illness management concepts

\begin{tabular}{ll}
\hline PACIC Concepts & \multicolumn{1}{c}{ Concept Description } \\
\hline Patient Activation (items 1-3) & $\begin{array}{l}\text { Actions that solicit patient input and } \\
\text { involvement in decision-making } \\
\text { Actions that organize care and provide } \\
\text { information to patients to enhance their } \\
\text { Delivery System Design/Decision Support } \\
\text { (items 4-6) }\end{array}$ \\
$\begin{array}{l}\text { understanding of care } \\
\text { Acquiring information for and setting } \\
\text { specific, collaborative patient-specific goals } \\
\text { (items 7-11) }\end{array}$ & $\begin{array}{l}\text { Considering potential barriers and the } \\
\text { patient's social and cultural environment in } \\
\text { Problem-Solving (items 12-15) }\end{array}$ \\
$\begin{array}{l}\text { making care plans } \\
\text { Arranging care that extends beyond office- } \\
\text { bollow-Up/Coordination (items 16-20) } \\
\text { making proactive contact with patients and } \\
\text { other providers to assess progress and } \\
\text { coordinate care }\end{array}$ \\
\hline
\end{tabular}


practice, clinic schedule, number of KTR patients, use of information technology in clinical practice, use of evidence-based guidelines, and preferred methods of continuing education in transplant. The demographic questionnaire was adapted from questionnaires used in CKD and primary care.

\section{ACIC Survey}

Primary Quantitative Outcome Data. The primary quantitative outcome data were collected using the Assessment of Chronic Illness Care Version 3.5 (Appendix E). The extent to which the care delivered by the provider was consistent with the elements of the CCM was measured with the ACIC (Bonomi et al., 2002). The ACIC is comprised of 34 questions divided into seven sections which include one section for each of the six elements of the Wagner (1998) model and a seventh section to assess the integration of these elements, which recognizes their independence. Each item was scored on a 0-11 scale and provided subscale scores for each of the $6 \mathrm{CCM}$ components and an integration score which describes the integration of CCM elements into daily patient care and a summary score for the entire instrument. A score from 0-2 represents "limited or no support for chronic illness care;" a score from 3-5 represents "basic or intermediate support;" a score from 6-8 represents "advanced support;" and a score from 9-11 represents "optimal or comprehensive, integrated care for chronic illness" (MacColl Center, n.d.). A detailed description of the ACIC concepts is provided in Table 3-2.

Psychometric Properties. Initial testing of the ACIC as a quality improvement tool included (a) pre-post, self-report ACIC data from four organizational teams enrolled in a 13month quality improvement project focused on care for chronic illness and (b) independent faculty ratings of team progress at the end of the collaborative. Paired $t$-tests were used to evaluate the sensitivity of the ACIC to detect improvements (Bonomi et al., 2002). According to Cramm, Strating, Tsiachristas, and Nieboer (2011), the ACIC was both a valid and reliable instrument. In terms of the instrument's construct validity, confirmatory factor analysis revealed goodness of fit with the items. In terms of the instrument's reliability, the analysis revealed acceptable internal consistency based on the Cronbach's alpha of all the subscales ranging from an internal consistency of (alpha $=.85$ [community linkages] - .97 [summary score]).

\section{Qualitative Components}

\section{KTR Sample}

The researcher prepared a focus group guide (Appendix F) to evaluate the participants' experiences concerning topics such as personal experiences with their kidney disease, their knowledge and experiences about integrated care and the care group they are a part of, the barriers and facilitators they encountered to their care, and health outcomes they achieved and how the former may have affected the latter. The KTR sample participated in focus group interviews where they shared their experiences as a KTR. The group interaction can act as a catalyst to activate forgotten details about and generate unique insights into factors that influence behavior and opinions of the shared experiences with the healthcare system and community that influence their ability to receive chronic care illness management (Green \& Boulware, 2016). 
Table 3-2. Description of chronic illness management concepts

\begin{tabular}{|c|c|}
\hline ACIC Concepts & $\begin{array}{c}\text { Concept Descriptions } \\
\end{array}$ \\
\hline Organization of Healthcare (items 1-6) & $\begin{array}{l}\text { Refers to the prioritization of chronic illness } \\
\text { management through healthcare system } \\
\text { design, leadership, and policies }\end{array}$ \\
\hline Community Linkages (items 7-9) & $\begin{array}{l}\text { Refers to mobilizing community resources to } \\
\text { meet the needs of consumers and encourage } \\
\text { consumer participation }\end{array}$ \\
\hline Self-Management Support (items 10-13) & $\begin{array}{l}\text { Refers to the effective self-management } \\
\text { support strategies that include assessment, } \\
\text { goal-setting, action-planning, problem- } \\
\text { solving, and follow-up and emphasizes the } \\
\text { person's central role in their health }\end{array}$ \\
\hline Decision Support (items 14-17) & $\begin{array}{l}\text { Refers to the promotion of clinical care that is } \\
\text { consistent with scientific evidence and } \\
\text { consumer preferences }\end{array}$ \\
\hline Delivery Systems Design (items 18-23) & $\begin{array}{l}\text { Refers to transforming a system that is } \\
\text { essentially reactive-responding mainly when } \\
\text { a person is sick-to one that is proactive and } \\
\text { focused on keeping a person as healthy as } \\
\text { possible }\end{array}$ \\
\hline Clinical Information Systems (items 24-28) & $\begin{array}{l}\text { Refers to the organization of patient and } \\
\text { population data to facilitate efficient and } \\
\text { effective care }\end{array}$ \\
\hline Integration of CCM (items 29-34) & $\begin{array}{l}\text { Assesses integration of elements of the } \\
\text { Chronic Care Model }\end{array}$ \\
\hline
\end{tabular}




\section{HCP Sample}

The researcher also developed a guide and cover letter for the HCP interview (Appendixes $\mathbf{G}$ and $\mathbf{H}$ ). The HCPs participated in individual in-depth interviews that provided them with an opportunity to engage in a candid discussion about their perceptions of the CCM elements and the influence of these elements on their ability to provide chronic illness management to KTRs. In-depth interviews allowed the HCPs to identify concerns or concepts that may not have been identified nor anticipated by the quantitative measurement.

\section{Data Collection, Management, and Analysis}

\section{Data Collection}

\section{KTR Collection Procedures}

Data collection for the patient participants, who received a KTR cover letter (Appendix I), took place between September 2015 and March 2016. Those participants who expressed interest in participation in KTR focus groups were scheduled for participation in one of three focus group interviews held on different days in a conference room at MUTI, which was familiar to all KTR participants. Qualitative and quantitative strand data collection was completed during a single study visit for these participants $(n=24)$. At the time of the interview, the consent form (Appendix A) was reviewed, and each participant signed the informed consent form, indicating that he or she read the study information and had the opportunity to ask questions, understood that participation in the research was voluntary and could be revoked at any time, and that he or she agreed to participate in the research which included audio-taping the interview. Quantitative data were collected prior to the commencement of the KTR focus group.

The focus groups were conducted by the researcher-moderator, who has been trained as a focus group moderator through the Burke Institute. The researcher-moderator was not a member of any participant's healthcare team. Each 90-minute focus group was guided by nine predetermined, open-ended questions. If the discussions led to different or unexpected relevant data, the researcher-moderator encouraged that discussion. Detailed notes were taken during the focus group to augment the audio tape with descriptions of any non-verbal communication that may have occurred. The notes included the respondent's characteristics; influence by other participants; context within which the comments were made; internal consistency or changes in opinion or influence by other participants; frequency and extensiveness of contribution in the discussion; and specificity of comments, such as from personal experience or hypothetical situations (Teddlie \& Tashakkori, 2009). The trustworthiness of the qualitative strand was maximized through member validation. At the end of each session, the researcher-moderator summarized comments made by participants. Participants were asked to revise, clarify, or add to these summarized comments. Additional comments were incorporated into the focus group data.

The focus group participants were given \$50 Visa gift cards at the conclusion of the session. KTRs who agreed to participate only in the survey portion of the study $(n=85)$ were 
given the option of receiving a hard copy of the survey or completing the survey telephonically. The IRB-approved consent was read to the participants, and confirmation that completion of the survey signifies consent to participate was obtained verbally. If the KTR chose to receive a paper copy of the survey, the consent was mailed with a return postage-paid return envelope. Upon completion of the survey, a \$25 Visa gift card was mailed to each participant.

\section{HCP Collection Procedures}

Quantitative data collection was completed by the volunteer HCP via a web-based survey or a paper survey. The HCPs were given the option of completing an electronic survey administered through Qualtrics (Qualtrics, Provo, UT) or a paper survey. Those who requested a paper survey had the option of receiving the letter of consent and the surveys in Word documents via email or hand delivery by the PI. Waiver for signed documentation of consent for HCP was obtained from the IRB. Paper surveys were returned via secure facsimile to the PI's private office, email, or picked up by the PI upon HCP request. Upon completion of the survey, a $\$ 25$ Visa gift card was mailed to the address indicated by the HCP on the survey.

A one-on-one interview was held with those providers who agreed to participate in the qualitative portion of the study. One-on-one interviews took place in the HCP's personal office or at another mutually agreed upon location. The PI, a registered nurse who has clinical experience in a community nephrology clinic and in acute post-transplant patient care, conducted the HCP interviews. No interviews were conducted with any HCPs with whom the researcher worked in clinical practice. Pre-determined, open-ended questions guided the 60-minute interview; however, HCP participants were encouraged to discuss additional topics that they believed to be important to the research topic. Permission to audio tape was requested and notes were taken during the interview. The PI recorded detailed notes that included personal perceptions and reflections following each interview. For HCPs who also participated in individual interviews, their participation was deemed consent and an additional \$25 gift card was provided.

\section{Data Analysis}

The data analysis procedures in this convergent parallel design study were as follows: (a) independent quantitative and qualitative analytic procedures; (b) merger of the quantitative and qualitative portions occurred by comparing, contrasting, and synthesizing results; and (c) areas of convergence and divergence were identified. For data collected from both KTRs and HCPs, the qualitative analysis was completed before the quantitative to prevent knowledge of quantitative outcomes from influencing the qualitative thematic analysis. Finally, the results from each subsample were compared and contrasted, and the convergence or divergence of perceptions of chronic illness management between the two groups were identified and discussed, with implications for practice and future research needs identified. The specific analytical strategy that was used for both the quantitative and qualitative data are discussed in this section. 


\section{Quantitative Analysis}

Prior to data collection, the researcher created a codebook containing a reproduction of each question, with numerical codes assigned for each categorical and open-ended variable within the demographic questionnaire. This process included the identification of the question using SAS 9.4 software naming rules (Delwiche \& Slaughter, 2011) and its sequential position in the instruments. All raw data were coded according to numeric values with dummy codes created for dichotomous variables such as living or cadaver organ and variables such as other chronic conditions delineated within the codebook and entered into an Excel spreadsheet by the researcher. The researcher verified all entries twice.

The study design was cross-sectional, with the quantitative variables of interest being the summary and subscale scores of the PACIC and ACIC. SAS 9.4 statistical software was used for quantitative analyses. Total and subscale scores across both samples were examined. Distribution curves were assessed visually by histograms and box plots. Goodness of fit scores using Kolmogorov-Smirnov (K-S) and levels of skewness and kurtosis were examined. The KTR data were not found to have been sampled from underlying normal distributions. Generally, the KTR data were negatively skewed with skewness on the total and all subscales between -1.1 and -2.2. The K-S p-values for the total and subscales were $<.01$.

The HCP data were found to have been sampled from underlying normal distributions. The small sample size for HCP data presented significant challenges with analyses due to seven subscale and summary scores of interest. The skewness statistic on the total and subscales was between 0.17 and 0.5 , while the K-S p-values for the scales were all >.15.

Acknowledging the complications presented by the number of variables of interest among the two participant groups and the small sample sizes, coupled with potential violations of assumptions of normality, non-parametric tests were employed. Due to low cell counts in analyses of both the PACIC and the ACIC scores and to allow for a comparison of the two scales, the scores were collapsed to create dichotomized scores representing incorporation of the CCM concepts. This method of dichotomizing the scores for these scales has been used successfully in other research (Balbale, Etingen, Malhiot, Miskevics, \& LaVela, 2016; Jackson, Weinberger, Hamilton \& Edelman, 2008).

The PACIC scores were dichotomized, with scores less than 3 on the total and subscale scores representing that the patients perceived a low incorporation of CCM concepts in their transplant follow-up care and scores 3 and greater representing high incorporation of the CCM concepts in transplant follow-up care. Likewise, the ACIC scores were dichotomized to represent low to limited (0-5) and moderate to high (6-11) physician-perceived incorporation of CCM concepts in the care management of KTR.

Continuous data characteristics of the KTRs and HCPs were summarized, with means and standard deviations and medians and $25^{\text {th }}-75^{\text {th }}$ quartiles. Categorical data were reported as proportions. Chi- Square and Fisher's Exact tests, as appropriate, were used to determine if any significant associations existed between categorical independent variables and the scale scores. Continuous variables were analyzed using t-tests and Wilcoxon Rank Sum, as appropriate. An 
alpha of 0.05 was used to consider associations that might be relevant in the development of a comprehensive model of care for kidney transplantation.

\section{Qualitative Analysis}

Audio recordings from the three 90-minute, semi-structured focus group interviews were transcribed verbatim, producing 156 pages of text with KTR participants, while audio recordings from the five 60-minute, semi-structured individual interviews were transcribed verbatim, producing 80 pages of text. Participant identifiers were removed prior to analyzing the transcribed data. Due to the large volume of data produced, the researcher used the computer software NVivo 10 to organize and analyze the study themes.

Interview data were completely analyzed using thematic content analysis (Saldaña, 2012). The thematic content analysis process involved five phases. In the first phase, the researcher achieved familiarization with the data from each of the three focus groups and five interviews by reading interview transcripts multiple times. In the second phase, the researcher generated initial codes, labelling sections or chunks of text that were salient to the research aims within individual focus group sessions and between. In the third phase, the researcher created basic themes by grouping the codes which were similar in nature. In the fourth phase, the researcher grouped the basic themes under organizing themes that the researcher derived from the CCM concepts. In the fifth phase, the researcher further grouped the basic themes as barriers or facilitators to receiving or providing care that is congruent with the CCM. In the following section, the researcher describes the data analyses used for each aim and research question.

\section{Aims and Research Questions}

\section{Kidney Transplant Recipient Aims and Research Questions}

\section{Quantitative Aims}

Specific Aim One. Determine the degree to which patients believe the long-term care they receive following kidney transplantation aligns with the concepts of quality care as outlined in the CCM.

Research Question 1. How do patients rate their long-term care in the context of concepts identified in the CCM, as determined by the PACIC summary and the following subscale scores?

1) patient activation

2) delivery system design/decision support

3) goal-setting

4) collaborative problem-solving

5) follow-up and coordination 
Specific Aim One and its associated questions were addressed with descriptive statistics, including medians and IQR of subscale and summary scores on the PACIC survey.

Specific Aim Two. Determine whether demographic and clinical indicators as described above are associated with KTRs' self-reported receipt of long-term care that aligns with the CCM concepts of quality care (PACIC scores).

Research Question 2. Are demographic and clinical characteristics associated with PACIC summary and subscale scores?

Chi-Square or Fisher's Exact tests, whenever the expected counts in a 2 x 2 contingency table were less than 5 and in larger tables $20 \%$ of expected cell counts were less than 5 , were used to determine if any significant associations existed between categorical independent variables and the dichotomized scale scores. Wilcoxon Rank Sum was used for continuous variables to compare the dichotomized subscale and summary scores with continuous patient characteristics.

\section{Qualitative Aim}

Specific Aim Three. Develop a rich understanding of the transplant experience, including experiences with the larger healthcare system, access to care, coordination of care, relationships with providers, and lifestyle implications.

Research Question 3. How do the KTRs experience the transplant process?

Research Question 4. What barriers and facilitators to quality long-term care are experienced?

The researcher analyzed the focus group data using constant comparative analysis to create codes, categories, and themes as described previously.

\section{Healthcare Provider Aims and Research Questions}

\section{Quantitative Aims}

Specific Aim Four. Determine the degree to which HCPs believe the long-term care provision to their kidney transplant patients aligns with the concepts of quality care as outlined in the chronic care model.

Research Question 5. How do HCPs rate their overall provision of long-term care in the context of concepts identified in the CCM, as determined by the ACIC summary score and the following subscale scores? 
1) organization of the healthcare system

2) community linkages

3) self-management support

4) decision support

5) delivery system design

6) clinical information system

7) integration of the CCM components into the delivery of care

Specific Aim Four and its associated questions were addressed with descriptive statistics, including medians and IQR of subscale and summary scores on the ACIC survey were produced.

Specific Aim Five. To determine whether demographic and clinical practice patterns are associated with HCPs' self-reported delivery of long-term, follow-up care that aligns with the CCM concepts of quality care.

Research Question 6. Are demographic and practice patterns associated with summary and subscale scores on the ACIC in this sample of HCPs?

Student's $t$-test and Wilcoxon Rank Sum, as appropriate, were used for continuous variables to compare the binary levels of the subscale and summary scores across the physician and practice characteristics.

\section{Qualitative Aim}

Specific Aim Six. Develop a rich understanding of the HCPs' experiences with provision of long-term chronic illness care for KTRs, including experiences with the larger healthcare system, access to care, coordination of care, relationships with patients, and personal practice implications.

Research Question 7. How do the HCPs experience the care of transplant recipients?

Research Question 8. What barriers and facilitators to the provision of quality long-term care are experienced?

The researcher analyzed the interview data using constant comparative analysis to create codes, categories, and themes as described above.

\section{Summary}

The researcher adopted a mixed methods research approach, which involves the strategic integration of quantitative and qualitative methodologies in a single study (Zhang \& Creswell, 2013). A convergent parallel mixed methods design was used in the current cross-sectional, correlational study of chronic illness management in the follow-up care of KTRs. Quantitative and qualitative data were collected separately, but in parallel fashion, from both KTRs and HCPs. 
Surveys were administered and interviews and focus groups were conducted with the participants. The researcher collected quantitative data using PACIC and ACIC survey questionnaires. During individual and focus group interviews with transplant recipients and nephrologists, respectively, the participants provided their perspectives in detail and a more comprehensive understanding of the perceptions of key stakeholders about the chronic illness management of KTRs.

The findings from quantitative and qualitative data were integrated in order to acquire a more complete description and explanation of both the KTRs' and the HCPs' experiences in long-term, follow-up care. For the qualitative data, the researcher used NVivo 10 to manage, organize, and analyze data from the interviews and focus group discussions. Lastly, the interpreted results of the two participant samples were integrated to describe the research phenomenon and identify opportunities for targeted interventions to improve long-term care following kidney transplantation. The researcher will discuss the results of the quantitative and qualitative strands of each sample and the integration of results from both KTRs and HCPs in the following chapters. 


\section{CHAPTER 4. RESULTS}

The purpose of this study was to determine, from the perspectives of the KTR and HCP, regarding chronic illness management as well as the barriers and facilitators to care as patients transition to the care of their community providers. In order to answer the research questions stated previously, two convergent parallel studies were undertaken. The convergent parallel design of this mixed methods study dictated that the Quan and Qual findings were analyzed independently and then merged and interpreted for a more complete understanding of the phenomenon. The findings will be presented accordingly: 1) a description of the KTR participant sample will be provided followed by 2) the Quan results and discussion; 3) the Qual results for the KTR study sample will then be presented followed by the discussion; 4) a conclusion which integrates the KTR Quan + Qual results for the KTR study. The HCP participant sample results will follow and be presented in the same four-step manner.

\section{Description of KTR Participant Sample}

\section{Demographic Characteristics}

One hundred nine KTRs completed the PACIC survey, and 24 KTRs participated in focus groups. Table 4-1 shows the descriptive summary of the age of the participants at transplantation and at enrollment in the current study. Table 4-2 shows summaries of categorical measured demographic characteristics. Unlike the US national demographics of KTRs with majority male and Caucasian, $60 \%$ and $74 \%$ respectively, this KTR sample included almost equal proportions of men and women and more African Americans than Caucasians.

\section{Transplant and Personal Health Management Characteristics}

Clinical information gathered from chart review included etiology of kidney disease, source of kidney, and immediate or delayed graft function. Patient-reported information included number of kidney transplants, time on dialysis before first transplantation, number of chronic conditions requiring medical-management (other than CKD), and the number of hospitalizations. Personal health management information included smoking history, use of alcohol, and physical activity.

In this sample, a diagnosis of hypertension was the largest contributor to ESRD $(47,43 \%)$, and another $8 \%$ had an etiology of ESRD in the medical record of both hypertension and diabetes, as compared to $21 \%$ of the U.S transplant population reporting hypertension as the etiology of ESRD. Eighty-four percent of the participants had one kidney transplant, but $17 \%$ of the participants had more than one. Table 4-3 shows the frequency and percentage summaries of the clinical information. Table 4-4 shows the frequency and percentage summaries of personal health management characteristics. 
Table 4-1. Descriptive statistics for summaries of kidney transplant recipient continuous variables

\begin{tabular}{lcccccc}
\hline Characteristic & N & Median & Minimum & Maximum & Mean & SD \\
\hline Time on dialysis $^{\mathrm{b}}$ & $94^{\mathrm{a}}$ & 36 & 5 & 228 & 52.2 & 46.1 \\
Time with graft $^{\mathrm{c}}$ & 109 & 60 & 12 & 120 & 55.8 & 28.0 \\
Age at time of transplant & 109 & 51 & 11 & 74 & 50.17 & 13.60 \\
Current Age & 109 & 57 & 26 & 80 & 56.17 & 12.85 \\
\hline
\end{tabular}

Note. $\mathrm{SD}=$ Standard Deviation

${ }^{a}$ Thirteen KTRs had preemptive transplants and did not have dialysis treatments. Two data points were missing.

${ }^{\mathrm{b}}$ Time on dialysis refers to time spent in months on dialysis prior to first transplant.

${ }^{\mathrm{c}}$ Time with graft refers to time in months with the current graft. 
Table 4-2. Summaries of kidney transplant recipient demographic characteristics

\begin{tabular}{|c|c|c|c|}
\hline KTR Characteristic & $\mathbf{n}$ & Frequency & Percentage \\
\hline Gender & 109 & & \\
\hline Male & & 56 & 51.4 \\
\hline Female & & 53 & 48.6 \\
\hline Race & 109 & & \\
\hline Caucasian & & 48 & 44 \\
\hline African American & & 61 & 56 \\
\hline Partner Status & 109 & & \\
\hline Married/Partner & & 83 & 76.1 \\
\hline Single & & 26 & 23.9 \\
\hline Education & 109 & & \\
\hline No College & & 66 & 61 \\
\hline College/Associates Degree & & 43 & 39 \\
\hline Employment & 108 & & \\
\hline Employed & & 39 & 35.8 \\
\hline Unemployed & & 8 & 7.3 \\
\hline Retired & & 28 & 25.7 \\
\hline Disability & & 33 & 30.3 \\
\hline Income & 106 & & \\
\hline$<25,000$ & & 34 & 31.2 \\
\hline $25-50,000$ & & 54 & 49.5 \\
\hline $50,0001-80,000$ & & 14 & 12.8 \\
\hline$>80,000$ & & 4 & 3.7 \\
\hline Insurance & 108 & & \\
\hline Private & & 31 & 28.4 \\
\hline Public & & 77 & 70.6 \\
\hline
\end{tabular}


Table 4-3. Clinical and health characteristics of kidney transplant recipients

\begin{tabular}{|c|c|c|}
\hline Clinical/Health Characteristic & Frequency & Percentage \\
\hline \multicolumn{3}{|l|}{ Primary Disease } \\
\hline Diabetes & 9 & 8.3 \\
\hline Hypertension & 47 & 43.1 \\
\hline Glomerular Nephritis & 4 & 3.7 \\
\hline Polycystic Disease & 7 & 6.4 \\
\hline Other & 33 & 30.3 \\
\hline Diabetes \& Hypertension & 9 & 8.3 \\
\hline \multicolumn{3}{|l|}{ Source of Kidney } \\
\hline Deceased Donor & 80 & 73.4 \\
\hline Living Donor & 28 & 25.7 \\
\hline Missing & 1 & 0.9 \\
\hline \multicolumn{3}{|l|}{ Immediate/Delay Function } \\
\hline Immediate Graft Function & 96 & 88.1 \\
\hline Delay Graft Function & 13 & 11.9 \\
\hline \multicolumn{3}{|l|}{ Graft Current Functioning } \\
\hline Yes & 100 & 91.7 \\
\hline No & 9 & 8.2 \\
\hline \multicolumn{3}{|l|}{ Number of Kidney Transplants } \\
\hline 1 & 91 & 83.5 \\
\hline$>1$ & 18 & 16.5 \\
\hline \multicolumn{3}{|l|}{ Mode Dialysis } \\
\hline In-Center Hemodialysis & 72 & 66.1 \\
\hline Home Hemodialysis & 4 & 3.7 \\
\hline Peritoneal Dialysis & 15 & 13.8 \\
\hline Multiple Methods & 13 & 11.9 \\
\hline Missing & 5 & 4.6 \\
\hline \multicolumn{3}{|l|}{ Hospitalizations $\mathbf{s}^{\mathrm{a}}$} \\
\hline None & 62 & 58.5 \\
\hline $1-3$ & 35 & 33 \\
\hline $4-7$ & 9 & 8.5 \\
\hline Missing & 3 & .03 \\
\hline \multicolumn{3}{|l|}{ Number of Chronic Conditions ${ }^{b}$} \\
\hline No Chronic Conditions & 17 & 15.5 \\
\hline $2-3$ & 87 & 79.8 \\
\hline $4-5$ & 5 & 4.6 \\
\hline
\end{tabular}

Note. ${ }^{\text {a }}$ Hospitalizations were self-reported and related to their kidney transplant.

${ }^{\mathrm{b}}$ Chronic Conditions were self-reported, excluding CKD that needed medical care in an openend text box. 
Table 4-4. Personal health management characteristics of kidney transplant recipients

\begin{tabular}{lcc}
\hline Health Characteristic & Frequency & Percentage \\
\hline Smoking Status & & \\
Never Smoker & 70 & 64.2 \\
Prior Smoker & 28 & 25.7 \\
Smoker & 11 & 10.1 \\
Alcohol Use & & \\
$\quad$ Never & 75 & 68.8 \\
Less than weekly & 22 & 20.2 \\
1-4 drinks per week & 7 & 6.4 \\
5-7 drinks per week & 4 & 3.7 \\
Activity & & \\
Not Active: less than 1 hour per week & 37 & 33.9 \\
Somewhat Active: 1-3 hours per week & 52 & 47.7 \\
Active: 3-5 hours per week & 16 & 14.7 \\
Very Active: 5 hours or more per week & 4 & 3.7 \\
\hline
\end{tabular}

Note. ${ }^{\text {a }}$ One data point was missing.

${ }^{\mathrm{b}}$ Activity was self-reported and defined as activity that elevated heart rate. 


\section{KTR QUAN Results}

\section{Specific Aim One}

The first aim of the study was to determine the degree to which patients believe the longterm care they receive following kidney transplantation aligns with the concepts of quality care as outlined in the CCM.

Research Question 1. How do patients rate their long-term care in the context of concepts identified in the CCM, as determined by the PACIC summary and subscale scores?

The survey results of this sample of KTRs indicate very high overall satisfaction with the chronic illness management care that is aligned with the concepts of the CCM. The subscale and summary scores for the PACIC are provided in Table 4-5. It is important to note that 33 (30\%) KTRs rated their care as 5 out of 5 on all of the PACIC scales, indicating that they perceived their care to be perfectly aligned with the concepts of the CCM. The median PACIC summary score was 4. Survey questions 2 (Given choices about treatment to think about) and 8 (Helped to set specific goals to improve my eating or exercise) in patient activation and goal-setting subscales, respectively, received the rank of "Always" more often than any other questions on the survey by 92 (84\%) of KTR participants.

The question that received the lowest rank was found in the Follow-up/Coordination of Care subscale. Thirty-five percent (38) of KTR participants answered, "None of the time" to question 19: "Told how my visits with other types of doctors, like an eye doctor or other specialist, helped my treatment."

Additionally, because only 4 KTR participants assigned low scores on each of the PACIC subscales, the researcher undertook a subset review of these 4 KTRs to determine if there were any common characteristics. The subset analysis revealed 2 Caucasian men, 1 Caucasian female, and 1 AA female. They each received follow-up care from different physicians.

The major common characteristic was that 3 out of the 4 had more than one transplant and the remaining participant did not currently have a functioning graft. Thus, experiencing graft loss or dysfunction was the underlying characteristic of this subgroup. In contrast it cannot be ruled out that these 4 people misinterpreted the directions for completing the survey items.

\section{Specific Aim Two}

The second aim of the study was to determine whether demographic and clinical or personal health indicators were associated with KTRs' self-reported receipt of long-term care that aligns with the CCM concepts of quality care as determined by the PACIC survey.

Research Question 3. Are demographic and clinical characteristics associated with PACIC summary and subscale scores? 
Table 4-5. PACIC subscales and summary scores $(n=109)$

\begin{tabular}{lcc}
\hline PACIC Dimension & Median (25\%-75\%) & Min-Max \\
\hline Patient Activation & $5(4-5)$ & $2-5$ \\
Delivery Systems Design/Decision Support & $5(4-5)$ & $2-5$ \\
Goal-Setting & $5(3-5)$ & $1-5$ \\
Collaborative Problem-Solving & $5(4-5)$ & $1-5$ \\
Follow-up/Coordination of Care & $4(3-5)$ & $1-5$ \\
Total Scale & $4(4-5)$ & $1-5$ \\
\hline
\end{tabular}

Note. Min=minimum, Max=maximum; these are the lowest and highest scores recorded by the kidney transplant recipients. 
The associations between categorical patient demographics, clinical characteristics, health management behaviors, and PACIC subscale and summary scores are provided in Table 4-6 through Table 4-13.

A comparison of PACIC scores for KTRs receiving one transplant and those receiving more than one was undertaken, but there were no statistically significant differences between the two groups. These data are not displayed.

The number of hospitalizations was significantly associated with four of the PACIC subscales Tables 4-8 through 4-11. A KTR who experienced 4-7 hospitalizations was 4 times more likely to perceive low implementation of patient activation and 7.3 times more likely to give low implementation scores on the collaborative problem solving subscale. Additionally, those who did not return to the same nephrologist who provided care prior to transplant were almost twice as likely to perceive low implementation of follow-up and coordination than those who returned to the same provider and 3 times more likely to give low implementation of CCM on the total score (Table 4-12 and 4-13). Lastly, of the categorical demographic and clinical characteristics, race was associated with follow-up/coordination, with Caucasian KTRs 1.7 times more likely to perceive low implementation of follow-up and coordination than African Americans. None of the personal health management characteristics were associated with the PACIC.

The continuous variables of current age, age at first transplant, time with current graft, and time on dialysis were also analyzed for associations between the dichotomized high and low scores on the PACIC survey. There was a significant difference between participants who spent more time on dialysis (months) and their counterparts. Those who spent more time on dialysis perceived lower alignment with the CCM concepts of the PACIC subscales: Patient Activation $(M d n=96$ vs 36, $p=.03)$; Delivery System/Decision Support ( $M d n=96$ vs $36 p=.02)$; and Problem-Solving $(M d n=108$ vs 36, $p=.04)$. Additionally, those KTR who have had their current graft longer $(M d n=72)$ perceived lower implementation of the CCM collaborative problemsolving concept as compared to those who perceived high integration $(M d n=48)$ of those concepts $(p=.04)$. Neither age variable was associated with the PACIC scores.

\section{KTR QUAN Discussion}

The PACIC survey scores indicate that the KTR participants perceive their chronic care management as highly aligned with the CCM concepts. The characteristics that were assessed to determine associations with the perceptions of CCM concept implementation in their care management were derived from other studies of patient satisfaction and the CCM. Few of the demographic or patient characteristics were found to have an association with the PACIC subscale or summary scores.

Given that improving care coordination was a cornerstone in the IOM's roadmap to improving quality of care, it is interesting that, as in other studies using the PACIC, (Glasgow, Nelson, Whiteside \& King, 2005; Noel et al., 2014) the Follow-up/Coordination subscale received the lowest scores. 
Table 4-6. Associations between kidney transplant recipient characteristics and the PACIC patient activation subscale

\begin{tabular}{|c|c|c|c|c|}
\hline $\begin{array}{l}\text { Demographic } \\
\text { Characteristics }\end{array}$ & $\mathbf{n}$ & $\begin{array}{c}\text { High Implementation } \\
\text { CCM n (\%) }\end{array}$ & $\begin{array}{c}\text { Low Implementation } \\
\text { CCM n (\%) }\end{array}$ & $\begin{array}{c}\mathbf{P} \\
\text { Value }\end{array}$ \\
\hline Hospitalizations $^{\mathbf{a}}$ & 109 & & & .004 \\
\hline None & & $58(89 \%)$ & $7(11 \%)$ & \\
\hline $1-3$ & & $28(80 \%)$ & $7(20 \%)$ & \\
\hline $4-7$ & & $5(56 \%)$ & $4(44 \%)$ & \\
\hline Chronic Conditions ${ }^{b}$ & 92 & & & .99 \\
\hline $2-3$ & & $73(84 \%)$ & $14(16 \%)$ & \\
\hline $4-7$ & & $5(100 \%)$ & $0(0 \%)$ & \\
\hline Return to & 107 & & & .22 \\
\hline \multicolumn{5}{|l|}{ Nephrologist ${ }^{\mathrm{c}}$} \\
\hline Yes & & $81(86 \%)$ & $13(14 \%)$ & \\
\hline No & & $9(69 \%)$ & $4(31 \%)$ & \\
\hline Partner Status ${ }^{d}$ & 109 & & & .99 \\
\hline Yes & & $69(83 \%)$ & $14(17 \%)$ & \\
\hline No & & $22(85 \%)$ & $4(15 \%)$ & \\
\hline Education $^{\mathrm{e}}$ & 109 & & & .64 \\
\hline No College & & $56(85 \%)$ & $10(15 \%)$ & \\
\hline College Degree/AD & & $35(81 \%)$ & $8(19 \%)$ & \\
\hline Gender $^{\mathrm{f}}$ & 109 & & & .12 \\
\hline Male & & $50(89 \%)$ & $6(11 \%)$ & \\
\hline Female & & $41(77 \%)$ & $12(23 \%)$ & \\
\hline Race $^{\mathrm{g}}$ & 109 & & & .61 \\
\hline $\mathrm{AA}$ & & $52(85 \%)$ & $9(15 \%)$ & \\
\hline Non-AA & & $39(81 \%)$ & $9(19 \%)$ & \\
\hline
\end{tabular}

Note. $\mathrm{AD}=$ Associate's degree, $\mathrm{AA}=\mathrm{A}$ frican American, $\mathrm{PACIC}=$ Patient Assessment of Chronic Illness Care

Fisher's Exact two-tailed test was used whenever the expected counts in a 2 x 2 contingency table were less than 5 and in larger tables $20 \%$ of expected cell counts were less than 5 .

${ }^{a}$ Hospitalizations were self-reported as those related to their kidney transplant.

${ }^{\mathrm{b}}$ Chronic Conditions were self-reported conditions other than CKD that require medical care.

${ }^{\mathrm{c}}$ Return to nephrologist who referred to transplant.

${ }^{\mathrm{d}}$ Partner Status denotes either married or with a partner.

e,f,g, Chi-Square test was used. Numbers in parentheses indicate row percentages. 
Table 4-7. Associations between kidney transplant recipients' characteristics and the PACIC delivery system design subscale

\begin{tabular}{|c|c|c|c|c|}
\hline $\begin{array}{l}\text { Demographic } \\
\text { Characteristics }\end{array}$ & $\mathbf{n}$ & $\begin{array}{c}\text { High Implementation } \\
\text { CCM n (\%) }\end{array}$ & $\begin{array}{c}\text { Low Implementation } \\
\text { CCM n (\%) }\end{array}$ & $\begin{array}{c}P \\
\text { Value }\end{array}$ \\
\hline Hospitalizations $^{\mathrm{a}}$ & 109 & & & .01 \\
\hline None & & $56(86 \%)$ & $9(14 \%)$ & \\
\hline $1-3$ & & $31(89 \%)$ & $4(11 \%)$ & \\
\hline $4-7$ & & $8(89 \%)$ & $1(11 \%)$ & \\
\hline Chronic Conditions $^{b}$ & 92 & & & .99 \\
\hline $2-3$ & & $75(86 \%)$ & $12(14 \%)$ & \\
\hline $4-7$ & & $5(100 \%)$ & $0(0 \%)$ & \\
\hline Return to Nephrologist ${ }^{\mathrm{c}}$ & 107 & & & .66 \\
\hline Yes & & $83(88 \%)$ & $11(12 \%)$ & \\
\hline No & & $11(85 \%)$ & $2(15 \%)$ & \\
\hline Partner Status $^{d}$ & 109 & & & .18 \\
\hline Yes & & $70(84 \%)$ & $13(16 \%)$ & \\
\hline No & & $25(96 \%)$ & $1(4 \%)$ & \\
\hline Education $^{\mathrm{e}}$ & 109 & & & .78 \\
\hline Less than college & & $58(88 \%)$ & $8(12 \%)$ & \\
\hline College Degree/AD & & $37(86 \%)$ & $6(14 \%)$ & \\
\hline Gender $^{f}$ & 109 & & & .57 \\
\hline Male & & $50(89 \%)$ & $6(11 \%)$ & \\
\hline Female & & $45(85 \%)$ & $8(15 \%)$ & \\
\hline Race $^{\mathrm{g}}$ & 109 & & & .77 \\
\hline AA & & $54(89 \%)$ & $7(11 \%)$ & \\
\hline Non-AA & & $41(85 \%)$ & $7(15 \%)$ & \\
\hline
\end{tabular}

Note. $\mathrm{AD}=$ Associate's degree, $\mathrm{AA}=\mathrm{African}$ American, $\mathrm{PACIC}=$ Patient Assessmnet of Chronic Illness Care

Fisher's Exact two-tailed test was used whenever the expected counts in a 2 × 2 contingency table were less than 5 and in larger tables $20 \%$ of expected cell counts were less than $5 .{ }^{*} \mathrm{Chi}-$ Square test was used. Numbers in parentheses indicate row percentages.

${ }^{a}$ Hospitalizations were self-reported as those related to their kidney transplant.

${ }^{\mathrm{b}}$ Chronic Conditions were self-reported conditions other than chronic kidney disease.

${ }^{\mathrm{c}}$ Return to nephrologist who referred to transplant.

${ }^{\mathrm{d}}$ Partner Status denotes either married or with a partner.

e, $\mathrm{f}, \mathrm{g}$ Chi-Square test was used. Numbers in parentheses indicate row percentages. 
Table 4-8. Associations between kidney transplant recipients' demographic characteristics and the PACIC goal-setting subscale

\begin{tabular}{|c|c|c|c|c|}
\hline $\begin{array}{l}\text { Demographic } \\
\text { Characteristics }\end{array}$ & $\mathbf{n}$ & $\begin{array}{c}\text { High } \\
\text { Implementation } \\
\text { CCM n (\%) } \\
\end{array}$ & $\begin{array}{l}\text { Low Implementation CCM n } \\
(\%)\end{array}$ & $\begin{array}{c}\mathbf{P} \\
\text { Value }\end{array}$ \\
\hline Hospitalizations $^{\mathbf{a}}$ & 109 & & & .01 \\
\hline None & & $40(62 \%)$ & $25(38 \%)$ & \\
\hline $1-3$ & & $26(74 \%)$ & $9(26 \%)$ & \\
\hline $4-7$ & & $4(44 \%)$ & $5(56 \%)$ & \\
\hline Chronic Conditions ${ }^{b}$ & 92 & & & .16 \\
\hline $2-3$ & & $56(64 \%)$ & $31(36 \%)$ & \\
\hline $4-7$ & & $5(100 \%)$ & $0(0 \%)$ & \\
\hline Return to Nephrologist ${ }^{\mathfrak{c}}$ & 107 & & & .54 \\
\hline Yes & & $32(84.2 \%)$ & $62(89.9 \%)$ & \\
\hline No & & $6(15.8 \%)$ & $7(10.1 \%)$ & \\
\hline Partner Status ${ }^{d}$ & 109 & & & \\
\hline Yes & & $52(63 \%)$ & $31(37 \%)$ & .64 \\
\hline No & & $18(69 \%)$ & $8(31 \%)$ & \\
\hline Education $^{\mathrm{e}}$ & 109 & & & .54 \\
\hline No College & & $44(67 \%)$ & $22(33 \%)$ & \\
\hline College Degree/AD & & $26(60 \%)$ & $17(40 \%)$ & \\
\hline Gender $^{\mathrm{f}}$ & 109 & & & .69 \\
\hline Male & & $37(66 \%)$ & $19(34 \%)$ & \\
\hline Female & & $33(62 \%)$ & $20(38 \%)$ & \\
\hline Race $^{g}$ & 109 & & & .32 \\
\hline AA & & $42(69 \%)$ & $19(31 \%)$ & \\
\hline Non-AA & & $28(58 \%)$ & $20(42 \%)$ & \\
\hline
\end{tabular}

Note. $\mathrm{AD}=$ Associate's degree, $\mathrm{AA}=\mathrm{African}$ American, PACIC=Patient Assessment of Chronic Illness Care

Fisher's Exact two-tailed test was used whenever the expected counts in a 2 x 2 contingency table were less than 5 and in larger tables $20 \%$ of expected cell counts were less than 5 .

${ }^{\text {a }}$ Hospitalizations were self-reported as those related to their kidney transplant.

${ }^{\mathrm{b}}$ Chronic Conditions were self-reported conditions other than chronic kidney disease.

${ }^{\mathrm{c}}$ Return to nephrologist who referred to transplant.

${ }^{\mathrm{d}}$ Partner Status denotes either married or with a partner.

e, f, g, Chi-Square test was used. Numbers in parentheses indicate row percentages. 
Table 4-9. Associations between kidney transplant recipients' demographic characteristics and the PACIC collaborative problem-solving subscale

\begin{tabular}{|c|c|c|c|c|}
\hline $\begin{array}{l}\text { Demographic } \\
\text { Characteristics }\end{array}$ & $\mathbf{n}$ & $\begin{array}{c}\text { High Implementation } \\
\text { CCM n (\%) }\end{array}$ & $\begin{array}{c}\text { Low Implementation } \\
\text { CCM n (\%) }\end{array}$ & $\begin{array}{c}\mathbf{P} \\
\text { Value }\end{array}$ \\
\hline Hospitalizations ${ }^{\mathbf{a}}$ & 109 & & & .001 \\
\hline None & & $61(94 \%)$ & $4(6 \%)$ & \\
\hline $1-3$ & & $31(89 \%)$ & $4(11 \%)$ & \\
\hline $4-7$ & & $5(56 \%)$ & $4(44 \%)$ & \\
\hline Chronic Conditions $^{b}$ & 92 & & & .99 \\
\hline $2-3$ & & $78(90 \%)$ & $9(10 \%)$ & \\
\hline $4-5$ & & $5(100 \%)$ & $0(0 \%)$ & \\
\hline Return to Nephrologist ${ }^{\mathfrak{c}}$ & 107 & & & .99 \\
\hline Yes & & $83(88 \%)$ & $11(12 \%)$ & \\
\hline No & & $12(92 \%)$ & $1(8 \%)$ & \\
\hline Partner Status ${ }^{d}$ & 109 & & & .48 \\
\hline Yes & & $75(90 \%)$ & $8(10 \%)$ & \\
\hline No & & $22(85 \%)$ & $4(15 \%)$ & \\
\hline Education $^{\mathrm{e}}$ & 109 & & & .99 \\
\hline No College & & $59(89 \%)$ & $7(11 \%)$ & \\
\hline College Degree/AD & & $38(88 \%)$ & $5(12 \%)$ & \\
\hline Gender $^{\text {f }}$ & 109 & & & .99 \\
\hline Male & & $50(89 \%)$ & $6(11 \%)$ & \\
\hline Female & & $47(89 \%)$ & $6(11 \%)$ & \\
\hline Raceg $^{\mathrm{g}}$ & 109 & & & \\
\hline AA & & $54(89 \%)$ & $7(11 \%)$ & .99 \\
\hline Non-AA & & $43(90 \%)$ & $5(10 \%)$ & \\
\hline
\end{tabular}

Note. $\mathrm{AD}=$ Associate's degree, $\mathrm{AA}=\mathrm{A}$ frican American, $\mathrm{PACIC}=$ Patient Assessment of Chronic Illness Care

Fisher's Exact two-tailed test was used whenever the expected counts in a 2 x 2 contingency table were less than 5 and when in larger tables $20 \%$ of expected cell counts were less than 5 . ${ }^{a}$ Hospitalizations were self-reported as those related to their kidney transplant.

${ }^{\mathrm{b}}$ Chronic Conditions were self-reported conditions other than chronic kidney disease.

${ }^{\mathrm{c}}$ Return to nephrologist who referred to transplant.

${ }^{\mathrm{d}}$ Partner Status denotes either married or with a partner.

e, f, g, Chi-Square test was used. Numbers in parentheses indicate row percentages. 
Table 4-10. Associations between kidney transplant recipients' demographic characteristics and the PACIC follow-up/coordination subscale

\begin{tabular}{|c|c|c|c|c|}
\hline $\begin{array}{l}\text { Demographic } \\
\text { Characteristics } \\
\end{array}$ & $\mathbf{n}$ & $\begin{array}{c}\text { High Implementation } \\
\text { CCM n (\%) }\end{array}$ & $\begin{array}{c}\text { Low Implementation } \\
\text { CCM n (\%) } \\
\end{array}$ & $\begin{array}{c}\mathbf{P} \\
\text { Value }\end{array}$ \\
\hline Hospitalizations $^{\mathbf{a}}$ & 109 & & & .87 \\
\hline None & & $42(65 \%)$ & $23(35 \%)$ & \\
\hline $1-3$ & & $22(63 \%)$ & $13(37 \%)$ & \\
\hline $4-7$ & & $5(56 \%)$ & $4(44 \%)$ & \\
\hline Chronic Conditions ${ }^{b}$ & 92 & & & .12 \\
\hline $2-3$ & & $54(62 \%)$ & $33(38 \%)$ & \\
\hline $4-5$ & & $5(100 \%)$ & $0(0 \%)$ & \\
\hline Return to Nephrologist ${ }^{\mathrm{c}}$ & 107 & & & .07 \\
\hline Yes & & $62(66 \%)$ & $32(34 \%)$ & \\
\hline No & & $5(38 \%)$ & $8(62 \%)$ & \\
\hline Partner Status ${ }^{d}$ & 109 & & & .26 \\
\hline Yes & & $50(60 \%)$ & $33(40 \%)$ & \\
\hline No & & $19(73 \%)$ & $7(27 \%)$ & \\
\hline Education $^{\mathrm{e}}$ & 109 & & & .23 \\
\hline No College & & $45(68 \%)$ & $21(32 \%)$ & \\
\hline College Degree/AD & & $24(56 \%)$ & $19(44 \%)$ & \\
\hline Gender $^{\mathrm{f}}$ & 109 & & & .33 \\
\hline Male & & $38(68 \%)$ & $18(32 \%)$ & \\
\hline Female & & $31(58 \%)$ & $22(42 \%)$ & \\
\hline Race $^{g}$ & 109 & & & .04 \\
\hline $\mathrm{AA}$ & & $44(72 \%)$ & $17(28 \%)$ & \\
\hline Non-AA & & $25(52 \%)$ & $23(48 \%)$ & \\
\hline
\end{tabular}

Note. $\mathrm{AD}=$ Associate's degree, $\mathrm{AA}=\mathrm{A}$ frican American, $\mathrm{PACIC}=$ Patient Assessment of Chronic Illness Care

Fisher's Exact two-tailed test was used whenever the expected counts in a 2 x 2 contingency table were less than 5 and in larger tables $20 \%$ of expected cell counts were less than 5 .

${ }^{a}$ Hospitalizations were self-reported as those related to their kidney transplant.

${ }^{\mathrm{b}}$ Chronic Conditions were self-reported conditions other than chronic kidney disease.

${ }^{c}$ Return to nephrologist who referred to transplant.

${ }^{\mathrm{d}}$ Partner Status denotes either married or with a partner.

e, f, g, Chi-Square test was used. Numbers in parentheses indicate row percentages. 
Table 4-11. Associations between kidney transplant recipients' demographic characteristics and the PACIC total scale score

\begin{tabular}{|c|c|c|c|c|}
\hline $\begin{array}{l}\text { Demographic } \\
\text { Characteristics }\end{array}$ & n & $\begin{array}{c}\text { High } \\
\text { Implementation } \\
\text { CCM n (\%) } \\
\end{array}$ & $\begin{array}{c}\text { Low Implementation CCM n } \\
(\%)\end{array}$ & $\begin{array}{c}P \\
\text { Value }\end{array}$ \\
\hline Hospitalizations $^{\mathbf{a}}$ & 109 & & & .15 \\
\hline None & & $54(83 \%)$ & $11(17 \%)$ & \\
\hline $1-3$ & & $29(83 \%)$ & $6(17 \%)$ & \\
\hline $4-7$ & & $5(56 \%)$ & $4(44 \%)$ & \\
\hline Chronic Conditions ${ }^{b}$ & 92 & & & .58 \\
\hline $2-3$ & & $71(82 \%)$ & $16(18 \%)$ & \\
\hline $4-7$ & & $5(100 \%)$ & $0(0 \%))$ & \\
\hline Return to Nephrologist ${ }^{\mathrm{c}}$ & 107 & & & .02 \\
\hline Yes & & $79(84 \%)$ & $15(16 \%)$ & \\
\hline No & & $7(54 \%)$ & $6(46 \%)$ & \\
\hline Partner Status $^{d}$ & 109 & & & .99 \\
\hline Yes & & $67(81 \%)$ & $16(19 \%)$ & \\
\hline No & & $21(81 \%)$ & $5(19 \%)$ & \\
\hline Education $^{\mathrm{e}}$ & 109 & & & .99 \\
\hline No College & & $53(80 \%)$ & $13(20 \%)$ & \\
\hline College Degree/AD & & $35(81 \%)$ & $8(19 \%)$ & \\
\hline Gender $^{\mathrm{f}}$ & 109 & & & .99 \\
\hline Male & & $45(80 \%)$ & $11(20 \%)$ & \\
\hline Female & & $43(81 \%)$ & $10(19 \%)$ & \\
\hline Raceg $^{g}$ & 109 & & & .47 \\
\hline $\mathrm{AA}$ & & $51(84 \%)$ & $10(16 \%)$ & \\
\hline Non-AA & & $37(77 \%)$ & $11(23 \%)$ & \\
\hline
\end{tabular}

Note. $\mathrm{AD}=$ Associate's degree, $\mathrm{AA}=\mathrm{African}$ American $\mathrm{PACIC}=$ Patient Assessment of Chronic Illness Care

Fisher's Exact two-tailed test was used whenever the expected counts in a 2 x 2 contingency table were less than 5 and in larger tables $20 \%$ of expected cell counts were less than 5 .

${ }^{a}$ Hospitalizations were self-reported as those related to their kidney transplant.

${ }^{\mathrm{b}}$ Chronic Conditions were self-reported conditions other than chronic kidney disease.

${ }^{\mathrm{c}}$ Return to nephrologist who referred to transplant.

${ }^{\mathrm{d}}$ Partner Status denotes either married or with a partner.

e, f, g, Chi-Square test was used. Numbers in parentheses indicate row percentages. 
Table 4-12. Comparison of dichotomized PACIC scores and time spent on dialysis with the perception of high or low implementation of CCM concepts in post-transplant followup care

\begin{tabular}{lccc}
\hline PACIC & $\mathbf{n}$ & Median $\left.^{\left(\mathbf{2 5}^{\text {th }} \mathbf{- 7 5}\right.} \mathbf{7}^{\mathbf{T h}}\right)^{\mathbf{a}}$ & P Value \\
\hline Patient Activation & & & \\
$\quad$ High Implementation & 83 & $36(18-84)$ & .034 \\
$\quad$ Low Implementation & 11 & $96(24-228)$ & \\
Delivery System & & & \\
$\quad$ High Implementation & 87 & $36(18-95)$ & .017 \\
$\quad$ Low Implementation & 7 & $96(60-228)$ & \\
Goal-Setting & & & \\
$\quad$ High Implementation & 63 & $36(19-84)$ & .129 \\
$\quad$ Low Implementation & 31 & $54(18-160)$ & \\
Problem-Solving & & & \\
$\quad$ High Implementation & 80 & $36(18-95)$ & .040 \\
$\quad$ Low Implementation & 24 & $108(36-136)$ & \\
Follow-up/Coordination & & & \\
$\quad$ High Implementation & 60 & $42(24-96)$ & .431 \\
$\quad$ Low Implementation & 34 & $36(17-62)$ & \\
Total Scale & & & \\
$\quad$ High Implementation & 80 & $36(18-96)$ & .174 \\
$\quad$ Low Implementation & 14 & $69(18-62)$ & \\
\hline
\end{tabular}

Note. PACIC=Patient Assessment of Chronic Illness Care. Time on dialysis represents the number of months the patient spent on dialysis prior to the transplant. Wilcoxon Rank Sums Test was used to assess the time spent on dialysis with the perception of high or low implementation of Chronic Care Model concepts in post-transplant follow-up care.

${ }^{a}$ Numbers in parentheses represent the first and third quartiles. 
Table 4-13. Comparison of dichotomized PACIC scores and time with current graft with the perception of high or low implementation of CCM concepts in post-transplant followup care

\begin{tabular}{lccc}
\hline PACIC & n & Median $\left(\mathbf{2 5}^{\text {th }} \mathbf{- 7 5 ^ { \text { Th } }}\right)^{\mathbf{a}}$ & P Value \\
\hline Patient Activation & & & \\
$\quad$ High Implementation & 91 & $60(25-72)$ & .316 \\
$\quad \begin{array}{l}\text { Low Implementation } \\
\text { Delivery System }\end{array}$ & 18 & $54(35-96)$ & \\
$\quad$ High Implementation & 95 & $60(27-84)$ & .378 \\
$\quad$ Low Implementation & 14 & $42(29-77)$ & \\
Goal-Setting & & & \\
$\quad$ High Implementation & 70 & $60(29-72)$ & .440 \\
$\quad$ Low Implementation & 39 & $48(26-84)$ & \\
$\begin{array}{c}\text { Problem-Solving } \\
\text { High Implementation }\end{array}$ & 97 & $48(26-72)$ & .038 \\
$\quad$ Low Implementation & 12 & $72(36-96)$ & \\
Follow-up/Coordination & & & \\
$\quad$ High Implementation & 69 & $60(30-77)$ & .348 \\
$\quad$ Low Implementation & 40 & $48(25-80.5)$ & \\
Total Scale & & & \\
$\quad$ High Implementation & 88 & $60(26.5-72)$ & .377 \\
$\quad$ Low Implementation & 21 & $48(35-96)$ & \\
\hline
\end{tabular}

Note. PACIC=Patient Assessment of Chronic Illness Care. Time with current graft is represented in months. Wilcoxon Rank Sums Test was used to assess the time with current graft and the perception of high or low implementation of Chronic Care Model concepts in post-transplant follow-up care.

${ }^{a}$ Numbers in parentheses represent the first and third quartiles. 
The continued lack of progress in coordination of care is an important barrier to chronic illness care management in the KTR population.

Although the KTRs in this sample appear to be low utilizers of transplant-related hospital services, with $60 \%$ reporting no hospitalizations, of those reporting hospitalizations there was a significant association between more hospitalizations and lower perception of the CCM concepts integrated in their care management. These results are unlike studies which found an association between hospital visit within the last 6 months and higher PACIC scores (Balbale et al., 2016; Rick et al., 2012). Interpretation of the current study results might be facilitated with knowledge of the recency of the self-reported hospitalization experiences.

Other studies have associated race with patient care satisfaction (Balbale et al., 2016; Carlin et al., 2012; Noel et al., 2016). The literature on race and patient satisfaction and outcomes are heterogeneous with some findings suggestive that patient satisfaction is higher when provider and patient are the same race, while others find that African Americans in general report higher satisfaction with care. However, some report White race associated with higher satisfaction. In this study, race was associated with only one PACIC subscale: Followup/Coordination of Care. Caucasian participants were 1.7 times more likely to perceive low implementation of the CCM concepts associated with follow-up/coordination. Because this sample of KTRs was predominantly African American and the providers were predominantly Caucasian this association might be a spurious finding, but warrants further investigation with regard to long-term outcomes.

It might be hypothesized that the loss of organ function would result in more negative patient ratings of their healthcare; however, longevity with a provider has been identified by other researchers as a positive influence on patient satisfaction scores (Bidaut-Russel et al., 2002; Carlin et al., 2012). This suggests that this might be a factor in the overall high scores of this patient sample. Almost all of this sample has been under the care of the same provider both prior to and after initial and subsequent transplants. Those who did not return to the care of their referring nephrologist were 1.8 times more likely to perceive low implementation of the followup and coordination concepts of the CCM and 3 times more likely to perceive low implementation of CCM concepts in the summary score of the PACIC. These results further support the influence of longevity with a provider on patient satisfaction. Perhaps, these findings suggest that more frequent communication between the provider and patient, regardless of the reason, is associated with higher perceptions of the quality of chronic care management. Other research suggests that the degree of provider-patient communication is an important indicator for improved outcomes and, in complex chronic conditions, is related to patient-coping strategies due to health literacy and efficacy (Carlin et al., 2012; Glasgow et al., 2005). The majority of these patients returned to the care of their nephrologist for long-term, follow-up care (88\%). Of those seeking long-term, follow-up care from a different provider, $62 \%$ reported their care was lacking in coordination, reinforcing the notion that longevity with the provider increases satisfaction with care.

Patient-related continuous variables of age, age at first transplant, time with current graft, and time on dialysis were analyzed as described above. In support of the outcomes in the original study of the PACIC scale (Glasgow et al., 2005) there was no association between the age 
variables and the PACIC scores. Other studies however have found associations between age and PACIC scores (Bidaut-Russel et al., 2002; Carlin et al., 2012). Time on dialysis was the continuous variable most associated with the PACIC. Although there is a lack of published information on transplant patient satisfaction, our findings that lower satisfaction is associated with longer time on dialysis is supported by a study of current dialysis patients which found that a shorter time on dialysis was associated with higher satisfaction scores (Dad et al., 2018). One possible explanation for these findings is that the KTRs who spend more time on dialysis enter the transplant experience less healthy, thereby having lower perception of quality care management.

Interestingly, those KTRs who had their current graft longer reported a lower implementation of collaborative problem solving in their care management. A search of the literature found no previous studies with similar findings, although some research indicates KTRs' expectations of their new life exceed reality, specifically that transplantation is not a "cure all" (Paraag, Nicola, \& Holly, 2016). Another hypotheses for this finding may be that perceived low levels of collaborative problem solving could be a factor of KTR health status. For instance, a relatively healthy recipient who has maintained their graft longer, would require fewer follow-up appointments, thereby the providers involvement in navigating the patients social and cultural barriers would be diminished. An additional explanation for this finding is that the KTRs who have maintained their graft longer are no longer Medicare eligible resulting in decreased satisfaction with the problem-solving construct as the provider is unable to assist in alleviating this barrier to care. Additionally, the longer a KTR has not had dialysis, the longer they have not had weekly access to multi-professional care which provided much opportunity for problem solving assistance.

\section{KTR QUAL Results}

\section{Specific Aim Three}

The third aim of the study was to develop a rich understanding of the transplant experience, including experiences with the larger healthcare system, access to care, coordination of care, relationships with providers, and lifestyle implications.

Research Question 4. How do the KTRs experience the transplant process?

\section{experienced?}

Research Question 5. What barriers and facilitators to quality long-term care are

After the survey was completed structured discussion questions were used to guide focus groups and to elicit a more complete understanding of the transplant experience in general and long-term, follow-up care specifically. During the focus groups, the participants discussed the perceived barriers and facilitators to follow-up care that are congruent with the concepts related to quality chronic illness management. The questions that guided this discussion are provided in Appendix F. The emerging themes that were identified inductively from the CCM concepts 
were: (a) knowledge, (b) care delivery, (c) community resources, (d) mental/emotional health, (e) support, (f) self-engagement, and (g) provider relationship. Finally, these themes were grouped as barriers or facilitators to care management that is congruent with the CCM.

\section{Barriers to Effective Care Management}

\section{Knowledge}

This theme centered on experiences and perceptions related to information and education provided before and after receiving a transplant. The identified categories that comprise this theme were related to lifestyle changes and functionality, which centered predominantly on medications.

Many participants discussed changes in weight and indicated that they were not prepared for the effects of steroids. Participant discussion included statements such as, "If I could change anything, yes, it would be the medication. As far as, let's start with the steroids. Eating out of control. Eating things that I wouldn't normally just crave for. And to find myself sometimes it's so out of control that I want to get out of bed late at night to find a store to get junk food. And that's a problem for me." Another person supported a lack of knowledge or preparedness for weight gain by adding, "I wasn't prepared for the medicine — eating out of control—get out of bed at night to go buy junk food."

Other individuals indicated a lack of knowledge about other comorbities associated with medications, such as, "Complications right from the start. I was pre-diabetic before transplant, but with the steroids, I'm now diabetic - I didn't know about that" and "My kidney is working fine, but it seems like the rest of my body is falling apart, didn't know about that virus you can get after transplant - til I got it." Another described contracting a fungal infection, "which will likely lead to the loss of the graft, without any instructions regarding the dangers of gardening or working in the soil," while another stated, "I've contracted Cryptococcus. Which, I don't, I think that takes me out of the possibility of getting another kidney. I don't know that for a fact at this point. I still have Cryptococcus and we're fighting it. Probably will for another year. And I feel like maybe the Cryptococcus was probably a possible reason I'm gonna lose that kidney." Still others pointed out that they were given information, but it was too general or the information is not processed. For example, one participant stated, "The information you ARE given is too general, we are not all the same, I want specific to my situation," while another said, "You hear about stuff like the medications and side-effects, but when it's all dumped on you it's a different story-30 pills a day." Yet another participant added, "I'm coming up on my third year as a recipient. And dealing with different side effects I'm just like the person that really don't like to complain. But, having so many different side effects, it causes me to complain a lot more than I normally would. Learning how to cope with different things that my body is going through-it woulda been good to know."

Beyond medications, the researcher noted that there was a perceived lack of information about necessary lifestyle changes. Diet, in particular, including the necessary increase in fluids, while a welcome change, was something KTRs determined was not widely discussed prior to 
transplant. One participant explained, "I was only drinking a cup of water a day on dialysis; then I find out after I got dehydrated- I was supposed to be drinking seven, eight, nine bottles of water a day with a transplant."

One somewhat surprising finding was that many participants did not know the etiology of their kidney disease. Some indicated that the doctors do not know, stating, "I don't know what caused it and they don't - they never been able to figure that out." Another participant explained, "I'm not for certain, but I know it runs in my family. I got uncles and brothers all on dialysismy brother always been afraid of transplant, but now he's gonna try it."

\section{Care Delivery}

Within each focus group, the participants identified that a lack of communication in general and lack of consistent information in particular between providers resulted in medical problems. These communication gaps were identified between physicians and others caring for the transplant patient (i.e., nursing and pharmacy).

One person recalled conflict between what the pharmacist thought was correct and what he had been told by the nephrologist, stating:

The biggest problem I had was there's some antibiotics that I can't take and I had one pharmacist kept, he kept trying to give it to me. And I said, 'I'm not taking that because then I'm going to be sick. I'm going to give up my entire kidney, rejection, you know.' He said, 'But we're going to try,' and I said, 'No, we're not going to try.'

Still, other participants pointed out a lack of communication between the transplant clinic and the community provider. Some had been instructed to return to the transplant clinic annually, yet others were not, as exemplified by this response: "Am I the only one that doesn't get invited to visit the transplant center annually? I get a call once a year and just the question is, 'How are you doing?' And I say, 'Good.' And they said, 'Well, OK, thank you for your time.' I don't actually come here to the clinic."

Participants who do return to the transplant clinic annually denoted discrepancy between what the community provider thought should be done and what was carried out at the transplant clinic. One participant stated:

But every time I come here. And I said my doctor is seeing about my kidney. And he don't tell me nothing that's wrong. And he do all kind of tests and stuff on me. And I went back and told him one time. And he said, 'There's nothing wrong with your kidney, why she want to do a biopsy?' I said, 'Well, I don't know.' And because I had a bad experience when she gave me, I mean when she did give me the biopsy. They stuck apunched a hole in my stomach. But I said, 'I come here every year and I obey what you all want to do. But I don't feel comfortable with you giving me a biopsy.

Still, others described the lack of communication with other providers who are not directly involved or educated in the care of kidney transplant recipients. One participant explained: 
I was having to be, in my home town of Cleveland, visiting my parents. All of a sudden, he [local doctor] found out that the blood was gone. Well, he put me right in - he put me in the hospital and everything in Cleveland. Well, we took it as, they were communicating with the doctors up here and found out they weren't. Well, they gave me some kind of medicine because I wound up with something. And even the pharmacy down there, they dropped the ball because the doctor that dealt with me and put me in the hospital gave me the medicine. Well, he didn't know to draw a Prograf level. And all of a sudden, I became toxic and almost lost the kidney because of it. Where I was thinking they were communicating with each other.

Others added that the lack of communication between providers is so bad that everything should be managed by one facility. One participant agreed: "It's so bad. To where I had to-I just switched everything from the Med over to Methodist because there was just so much confusion with my labs, with them being drawn in one place and then they look at them from over here." Another person added, "I think everything should be up under one system to where they able to pull whatever doctors you go to, and they're able to go into their system and see what they done."

\section{Community Resources}

Focus group participants discussed community resources, including the influence of healthcare policy, particularly the importance of Medicare, in the ability to adhere to the required care regimen.

Participants noted discrepancies between their individual experiences. One participant expressed, "After 3 years, my premiums went up on my medicines, I almost went bankrupt." Another stated that prior to transplant, they were never told the Medicare coverage would end, explaining, "I had no idea... I got a letter last week to tell me my Medicare will stop." Other participants indicated that they were able to maintain a disability status with the help of their physician, as seen in one participant's statement that, "I had to fight tooth and nail to keep me on it, with doctors and everyone writing letters." Still, other participants had not had such problems maintaining their disability status, with one participant explaining, "I got put on permanent disability, they just went ahead even though I don't have anything but the transplant." The discrepancy in experiences with Medicare and the complexity of the system led to the question, "Why they give you a million-dollar kidney and then let it die—government at work." Others pointed out that social workers were very involved in the care plan on dialysis and that assistance was important in the complex world of insurance and pharmaceutical grants.

Some participants reported not speaking to a social worker since transplant, noting that this communication was more of a formality than real help. One participant explained, "I just had them to offer me some information, some kinda brochures." Another participant stated, "I mean, I think the last social worker that I just spoke to was the one at the dialysis center when I would go of my monthly check up [prior home hemodialysis]." Another managed to find information through people not directly involved in their care, stating, "My daughter she deals with social workers so if I have a problem she'll be there asking what to do for me." 
Beyond the importance of healthcare policy and insurance, there was discussion of the importance of other providers who were involved in their care prior to transplant, which included pharmacists, dieticians, and support groups. One participant expressed this concern, "They come in there while you all doped up and tell you about this drug and that then you don't remember none of it." Others recognized that they had to hear the information more than once, with some participants perceiving that they had a better experience than others with community resources. One participant explained, "My community nephrologist, he schooled me, he tell me everything and get dietician to talk to me after I got my kidney." Others discussed the importance of diet to graft maintenance, but not all had been given a guide for the appropriate diet. One participant said, "I haven't seen a dietician since I was on dialysis, but I figured that renal diet kept me off dialysis for a long time, so I'm gonna stick to that." In contrast, some participants were told what to eat: "I just went away from fried food. So, everything I eat is baked and everything I eat is vegetables, frozen vegetables. Cause ain't supposed to eat fresh vegetables cause the soil got bacteria." Participants spoke of the positive experience with the focus group and how much they learned just talking to each other.

Some KTRs mentioned that they had received information about a support group at the transplant center, but the meeting times were inconvenient. Other participants stated that they would be interested in virtual support groups. None of the participants were aware of the resources such as dietary guidelines, support groups, or educational webinars provided by the American Association of Kidney Patients.

\section{Mental/Emotional Health}

The participants highlighted the emotional burdens of transplant, but were quick to point out that they were grateful for their kidney and hoped never to return to dialysis. Many discussed the constant stress related to multiple factors, but there is a predominant fear of losing the kidney which causes stress and anxiety. Statements such as these illustrate the level of fear that the majority of participants described: "I'm terrified that I'll do something wrong-I don't want dialysis again," "You hear rejection, rejection, rejection in your sleep," and, "I'm a complete germaphobic, I feel like we should live in a bubble."

Other participants also discussed the stress related to financial burdens, with one man stating, "I want to take care of my family, my wife was the sole breadwinner while I was on dialysis, but now we got even more expenses - we could be bankrupt." Another added, "We weren't even told about Medicare ending til we were checking out of the hospital-I don't know what we will do, but it sure gets you down."

Still other KTRs pointed to depression related to the lifestyle and medication adherence. One participant expressed, "One pill to take care of what that pill caused and the cycle keeps repeating - it can set you in a state of depression." Some cited that the lifestyle with a transplant is restrictive and can be depressing, with one participant explaining, "Even though I can travel now, I can't have a drink. We went down to the islands and I not a drinker, but can't have alcohol, can't eat fresh fruit and vegetables, can't get tattoos, all that. It's a lot a strict stuff. It can make you tired and depressed sticking to it." 


\section{Facilitators to Effective Care Management}

\section{Support}

The majority of focus group participants explained the importance of having a support person who engages with them from the transplant work-up through long-term, follow-up care. When discussing support, one participant stated, "You are at a disadvantage if you don't have anyone with you, someone who gets the education too, because there is so much to think about." The need to have a strong support system was reiterated throughout the focus groups through the following statements:

"And another thing, you got to have a good family support. Your family makes a whole lot of difference."

"It is so important not to feel alone."

"Like I said, my mother's a pit-bull when it comes to my health. She knows all the nurses and everything. And she'll go, 'that's such and such.' And I had no clue who they are because, I'm sick and everything, but she knows all of them and who to call."

Others did who did not have the family support recognized the importance of support throughout the process. One participant expressed, "That was something I didn't have because my mother was my donor. And she was coming in from out of town. And my father really isn't in my life, he was physically there, but_-it's not like he's a care provider. He wasn't there for 23 years before. So, it's like - hard." Another explained, "Talking to other people who have been through this is important. I am on a chat room with other transplant recipients and that really helps me."

\section{Self-Engagement/Activation}

In addition to acknowledging the importance of an external support system, the participants identified the importance of self-engagement as a key factor in successfully navigating the transplant process. Overall, participants realized that they needed to be involved in their own care, with one participant explaining, "I think realizing that you, the patient, are responsible for communicating with your physicians is important." To expand upon this, participants discussed the importance of asking questions. One participant recommended, "Ask questions - you gonna live or you gonna die, you know your body better than they do." Others noted that they were all given a book of warning signs, but it was up to them to pay attention to those and make sure that someone listens to them. One participant noted, "I would say my experience with this transplant, I was real sick after the transplant and I kept telling, I think in 4 days and gaining 30 pounds I was so swollen that I couldn't even fit in my pajamas and I kept telling the doctor something is wrong with me. Oh no, you've got better creatinine. You know they look at it the kidney is working there's nothing else. But I kept on and ended up I had pancreatitis." Another participant noted, "I had warning signs, I was doing things, I had no business doing - I thought that is- warning might end in total destruction- and I had to stop." The majority of focus group participants pointed to the "gift of life" as the daily reminder to stay 
engaged in their health, with one participant stating, "My kidney came from a 5-year-old. Every time I think about doing this, drinking that, I know it's not as important as my gift."

One component of self-engagement/activation was the importance of attitude with a sense of perseverance. One participant explained, "You have to have a good attitude. The doctors told me one time, they said, 'We can only do — what we do, on $100 \%$ scale, we do $20 \%$, the $80 \%$ is up to you and your attitude." Another participant said, "You can either say, 'I'm going to do this, I'm going to survive, I'm going to do my best,' or you can [say], 'Poor little me.'”

\section{Provider Relationship}

The majority of the participants had been under the care of the same nephrologist since being diagnosed with chronic kidney disease, throughout dialysis, and the transplant process. Many participants discussed the importance of the relationship with the nephrologist and the staff. The following comments described these close personal relationships:

"I feel I have the best one in the city."

"I love him dearly. I hope he's at my wedding."

"It's important to have a doctor whose been knowing you, he can look at you and know you aren't right."

"My doctor is the best, my husband tell me, 'he can't know everything', but he does, he really does."

"My doctor, nephrologist, doctor that I had when I went on-when my kidney failed in 2011. And I love that doctor. Any doctor that would listen to me and can decide where I'm coming from or how I'm feeling, I'm with it."

"My doctor, she's more like my mom. So, if I'm not doing what I'm supposed to do. She's there, and I mean, she's - she's scolding. And I mean, she's going to make you get it right. And she's going to flat out tell you, what's what. Don't beat around the bush with me. Let me know off top, what am I facing? Or what are my chances? And I love that." "My nephrologist I see for my high blood pressure even before I started seeing him for my kidney. So, I've been seeing him since ' 85 . He practically knows everything about me. So, we have a great relationship."

"It's very important to be like a family unit with your doctor. It's like, very important, because that communication between you and your doctor, that's what's going to keep you alive. So, it's like a brother, father, and just listen. And most important thing, whatever they have - the most important thing, don't miss your doctor appointments. That's the key thing."

When asked to talk about the discrepancy between the accolades describing their nephrologist as the best and most knowledgeable and the problems regarding education and communication that they had discussed prior, most participants were quick to point out that those were problems related to someone else or the system and not their nephrologists.

Several participants also noted the importance of the support from the community nephrologists' office staff - many of whom they have also known for years - in comparison to their experience with dialysis personnel, where there is frequent staff turnover. One participant 
cited, "A lot of them are still there. The secretaries and everything. And it's a joy to see them. Because they took care of you so long. Even when you didn't know you were sick, they could look at you and tell you that you're not feeling well today. It was awesome to me." Another explained, "I know they're the ones calling me a lot of the times, when the doctor can't get to me or the nurse can't call or whatever, it's those receptionists or whatever, they're calling going, 'We need to do this. The doctor wants you to do this. You need to call me back and tell me.' And so, you form a bond with them too."

The participants' discussion of the transplant experience was predicated predominantly upon their comparisons to previous dialysis and the changes related to the transition from identifying as a dialysis patient versus a transplant patient. For example, the participants explained how their current medications, diet, integrated care, and general health compared to dialysis. All participants stated that no matter what problems they have now, they are grateful for their kidney, which they perceived as a "gift of life." Several participants described holding "birthday" celebrations on their transplant anniversary.

\section{KTR QUAL Discussion}

The QUAL strand of the KTR study sample sought to describe the lived experience of KTRs and the barriers and facilitators to receiving effective long-term, follow up care. There are few qualitative studies of KTRs in the U.S., and the dearth of subjective studies on the lived experience post-kidney transplant in the U.S. is of great concern. The large, diverse U.S. population coupled with the complex healthcare system present difficulties in drawing comparisons to other studies of the lived experience for KTRs, which to date have mostly been conducted in countries with a universal health system.

The overall lived experience described by KTR focus group participants was one of change, including significant daily lifestyle changes, new medical concerns, fear of losing the kidney, and new medical expenses. The descriptions of their lives as a transplant recipient was provided in comparison to the life they knew as a dialysis patient. Fear of losing the graft and a comparison to life as a dialysis patient are supported by a recent survey of U.S. KTRs (Tucker et al., 2019). Similar findings were noted in an Australian study of stressors and coping among KTRs (Low et al., 2017).

Additionally, a lack of coordination of care and misinformation between providers was noted as a major barrier to receiving effective long-term care management, resulting in errors and increased expenses. It has been posited in prior studies that the complex U.S. healthcare and payer systems might explain the lagging U.S. outcomes post kidney transplantation in crosscountry outcome comparisons (Ojo, 2013).

This study sample highlights the importance of self-management and the support of family in successful navigation of the healthcare system. Understanding the complicated nuances of insurance policies and medication coverage were the primary discussion points within the topic of community resources. Interactions with multidisciplinary staff in the dialysis units from 
nurses, social workers and dieticians, along with their "shift" friends, were often mentioned as positive experiences.

Despite any negative comments related to the healthcare system, the participants were quick to indicate how grateful they were to have received a transplant and to have the opportunity to live and travel without the restraints of planning transient dialysis treatments. Interestingly, there was little mention of re-entering the work force. This was somewhat surprising given prior research associating employment status post-transplant with improved quality of life and reduced morbidity and mortality. Several factors in this group of participants might explain the limited discussion of employment status as an influential aspect to their longterm care management. Years spent on dialysis might impact the KTRs employment prospects, as necessary skill sets might have changed. Additionally, several mentioned receiving disability coverage for other conditions.

The overwhelming majority stated that they have full confidence in their nephrologists' knowledge and genuine concern for them and overall the criticisms related to their care were not attributed to the nephrologist, but instead were attributed to unnamed others or the healthcare system in general. Based upon the high patient survey scores, it would be possible to hypothesize that the focus group results would not identify any barriers to CCM implementation. Previous researchers, however, have indicated that the association of self-management support, which includes patient education, and patient satisfaction reach a point of diminishing returns. Patient satisfaction has been positively associated with increased self-management support in less complex healthcare, such as management of a singular chronic condition, yet it is negatively associated in more complex care such as in kidney transplants. It is possible that KTRs who identified stress, fear, and guilt as management problems might be ill-equipped to manage any additional distress that comes with more explicit knowledge of their complex medical condition. Another possible explanation is that KTRs believe that the majority of the outcome is dictated by the organ received or factors beyond their control, diminishing the influence of their self-care behaviors on outcomes. Any of these possible explanations for the moderately divergent results between the PACIC and the focus group data support the importance of the patient-provider relationship.

\section{KTR QUAN + QUAL Integration}

Past research has indicated that certain demographic characteristics - such as age, gender, race and education - are associated with patient satisfaction and perception of quality of care (Jackson, Chamberlin, \& Kroenke, 2001). In this study, very few of the demographic characteristics which previous research has associated with patient satisfaction with care, were found to correlate with the PACIC scores. The discrepancy might be attributable to the fact that most prior research has focused on primary care rather than on complex patient health such as kidney transplantation.

The PACIC scores overall were supportive of high satisfaction with the care received. Despite the overall high scores reported on the PACIC, these were tempered by the focus group results which identified barriers or weaknesses in their chronic care management. However, the 
major barriers identified were unrelated to direct patient care provided by the nephrologist. As the PACIC survey sought information related to the provider, this might explain the incongruent results between the survey and the focus groups. Table 4-14 displays a comparison of the PACIC subscale scores and KTRs qualitative themes.

Ironically, but not surprisingly, medication was identified as a major barrier. Despite the necessity of immunosuppressive drugs to maintain the graft, they were associated with financial concerns, multiple comorbidities including overweight/obesity, and infections. Medication has long been identified as a major barrier to therapeutic adherence following kidney transplantation $\mathrm{i}$ and continues to be identified in recent studies (Cossart, Staatz, Campbell, Isbell, \& Cotrell, 2019; Ndemra \& Bhengu, 2017; Peterson, O’Rourke \& Thornton, 2018). The QUAL results found medication as part of the narrative in multiple barrier a priori and sub-themes such as knowledge, mental/emotional health, and community resources. The influence of the provider relationship on patient perception of chronic care management was evident in the focus groups. Although focus group participants described a lack of communication and coordination in their care management, as was also indicated in the PACIC scores, the focus group results did not directly attribute lack of coordination to the nephrologist. Likewise, the HCPs were not identified directly in any of the barrier themes within the PACIC subscales.

To the contrary, the providers were identified as facilitators to quality chronic illness management care in each of the PACIC subscales even when identifying barriers that the HCP might seemingly facilitate. For instance, lack of patient specific knowledge- even knowledge of the etiology of ESRD- was identified as a barrier to receiving quality care management. Although, the Agency for Healthcare Research and Quality (AHRQ) in their 2017 National Healthcare Quality and Disparities Report found that 33\% of hospital discharged patients did not know their diagnosis, indicating a lack of knowledge regarding health status is widespread, these findings are troubling and warrant further investigation.

Interestingly, the number of hospitalizations was the variable associated with more of the subscale scores, in that those who reported hospitalizations perceived lower implementation of CCM concepts. This finding was counter to some other studies using the PACIC survey. Perhaps, in this patient population, hospitalizations are often not in hospitals where the nephrologist exercise privileges. Perhaps the KTRs perceive that education is not the responsibility of the nephrologist, rather other team members such as social workers and case managers.

In other studies, longevity or a sustained relationship with a provider has been identified as a primary ingredient in patient satisfaction and quality outcomes. The findings in this study support this previous research. The majority of KTRs returned to the care of their long-time nephrologist and it was the only variable associated with the total PACIC scale. In the focus groups, many participants reported the nephrologist to be like family.

The term 'fictive kin' has been used by anthropologists and ethnographers to describe familial-type relationships between people who do not have a consanguineal or legal relationship, often in studies of African Americans. Although these relationships have been 
Table 4-14. Comparison of QUAN PACIC scores and QUAL focus group data

\begin{tabular}{|c|c|c|c|}
\hline PACIC Subscales & $\begin{array}{l}\text { PACIC } \\
\text { Scores } \\
\text { Mean } \\
(\text { SD) }\end{array}$ & $\begin{array}{c}\text { Barriers }^{\mathrm{b}} \\
\text { Qualitative Themes }\end{array}$ & $\begin{array}{c}\text { Facilitators }^{c} \\
\text { Qualitative Themes }\end{array}$ \\
\hline $\begin{array}{l}\text { Patient Activation: } \\
\text { Actions that solicit } \\
\text { patient input \& } \\
\text { involvement in } \\
\text { decision making }\end{array}$ & $\begin{array}{l}4.35 \\
(.99)\end{array}$ & $\begin{array}{l}\text { Knowledge: } \\
\text { "The information you ARE } \\
\text { given is too general, we are } \\
\text { not all the same, I want } \\
\text { specific to my situation," } \\
\text { Community Resources } \\
\text { "I haven't seen a dietician } \\
\text { since I was on dialysis, } \\
\text { Mental/Emotional Health } \\
\text { "One pill to take care of } \\
\text { what that pill caused and the } \\
\text { cycle keeps repeating-it } \\
\text { can set you in a state of } \\
\text { depression." }\end{array}$ & $\begin{array}{l}\text { Self-Engagement: } \\
\text { "Ask questions-you gonna live } \\
\text { or you gonna die, you know } \\
\text { your body better than they do." } \\
\text { Provider Relationship: } \\
\text { "It's very important to be like a } \\
\text { family unit with your doctor. } \\
\text { It's like, very important, } \\
\text { because that communication } \\
\text { between you and your doctor, } \\
\text { that's what's going to keep you } \\
\text { alive. So, it's like a brother, } \\
\text { father, and just listen. And most } \\
\text { important thing, whatever they } \\
\text { have-the most important thing, } \\
\text { don't miss your doctor } \\
\text { appointments. That's the key } \\
\text { thing." } \\
\text { Support person: } \\
\text { "You are at a disadvantage if } \\
\text { you don't have anyone with } \\
\text { you, someone who gets the } \\
\text { education too because there is } \\
\text { so much to think about." }\end{array}$ \\
\hline $\begin{array}{l}\text { Delivery System } \\
\text { Design: } \\
\text { Actions that } \\
\text { organize care \& } \\
\text { provide information } \\
\text { to patients }\end{array}$ & $\begin{array}{l}4.48 \\
(.86)\end{array}$ & $\begin{array}{l}\text { Care Delivery: } \\
\text { "It's so bad. To where I had } \\
\text { to-I just switched } \\
\text { everything from the Med } \\
\text { over to Methodist because } \\
\text { there was just so much } \\
\text { confusion with my labs } \\
\text { Community Resources: } \\
\text { "After } 3 \text { years, my } \\
\text { premiums went up on my } \\
\text { medicines, I almost went } \\
\text { bankrupt." } \\
\text { Mental/Emotional Health: } \\
\text { "I'm terrified that I'll do } \\
\text { something wrong." }\end{array}$ & $\begin{array}{l}\text { Provider Relationship: } \\
\text { "My nephrologist I see for my } \\
\text { high blood pressure even before } \\
\text { I started seeing him for my } \\
\text { kidney. So, I've been seeing him } \\
\text { since ' } 85 \text {. He practically knows } \\
\text { everything about me. So, we } \\
\text { have a great relationship." } \\
\text { "It's important to have a doctor } \\
\text { whose been knowing you, he } \\
\text { can look at you and know you } \\
\text { aren't right." } \\
\text { "My doctor is the best, my } \\
\text { husband tell me, 'he can't know } \\
\text { everything', but he does, he } \\
\text { really does." }\end{array}$ \\
\hline
\end{tabular}


Table 4-14. (Continued)

\begin{tabular}{|c|c|c|c|}
\hline PACIC Subscales & $\begin{array}{l}\text { PACIC } \\
\text { Scores } \\
\text { Mean } \\
(\text { SD) }\end{array}$ & $\begin{array}{c}\text { Barriers }{ }^{b} \\
\text { Qualitative Themes }\end{array}$ & $\begin{array}{c}\text { Facilitators }^{c} \\
\text { Qualitative Themes }\end{array}$ \\
\hline Goal-Setting & $\begin{array}{c}4.09 \\
(1.02)\end{array}$ & $\begin{array}{l}\text { Knowledge: } \\
\text { "I was only drinking a cup } \\
\text { of water a day on dialysis; } \\
\text { then I find out after I got } \\
\text { dehydrated I was supposed } \\
\text { to be drinking seven, eight, } \\
\text { nine bottles of water a day } \\
\text { with a transplant." } \\
\text { "having so many different } \\
\text { side effects, it causes me to } \\
\text { complain a lot more than I } \\
\text { normally would. Learning } \\
\text { how to cope with different } \\
\text { things that my body is going } \\
\text { through-it woulda been } \\
\text { good to know." }\end{array}$ & $\begin{array}{l}\text { Provider Relationship: } \\
\text { "My doctor, she's more like my } \\
\text { mom. So, if I'm not doing what } \\
\text { I'm supposed to do. She's there, } \\
\text { and I mean, she's - she's } \\
\text { scolding. And I mean, she's } \\
\text { going to make you get it right. } \\
\text { And she's going to flat out tell } \\
\text { you, what's what. Don't beat } \\
\text { around the bush with me. Let } \\
\text { me know off top, what am I } \\
\text { facing? }\end{array}$ \\
\hline Problem-Solving & $\begin{array}{l}4.49 \\
(.86)\end{array}$ & $\begin{array}{l}\text { Community Resources: } \\
\text { "I had to fight tooth and nail } \\
\text { to keep me on it (Medicare), } \\
\text { with doctors and everyone } \\
\text { writing letters." } \\
\text { "Why they give you a } \\
\text { million-dollar kidney and } \\
\text { then let it die-government } \\
\text { at work." }\end{array}$ & $\begin{array}{l}\text { Provider Relationship" } \\
\text { "My doctor, nephrologist, } \\
\text { doctor that I had when I went } \\
\text { on-when my kidney failed in } \\
\text { 2011. And I love that doctor. } \\
\text { Any doctor that would listen to } \\
\text { me and can decide where I'm } \\
\text { coming from or how I'm } \\
\text { feeling, I'm with it." } \\
\text { Support Person: } \\
\text { "Like I said, my mother's a } \\
\text { pitbull when it comes to my } \\
\text { health. She knows all the nurses } \\
\text { and everything. And she'll go, } \\
\text { 'that's such and such.' And I } \\
\text { had no clue who they are } \\
\text { because, I'm sick and } \\
\text { everything, but she knows all of } \\
\text { them and who to call." }\end{array}$ \\
\hline
\end{tabular}


Table 4-14. (Continued)

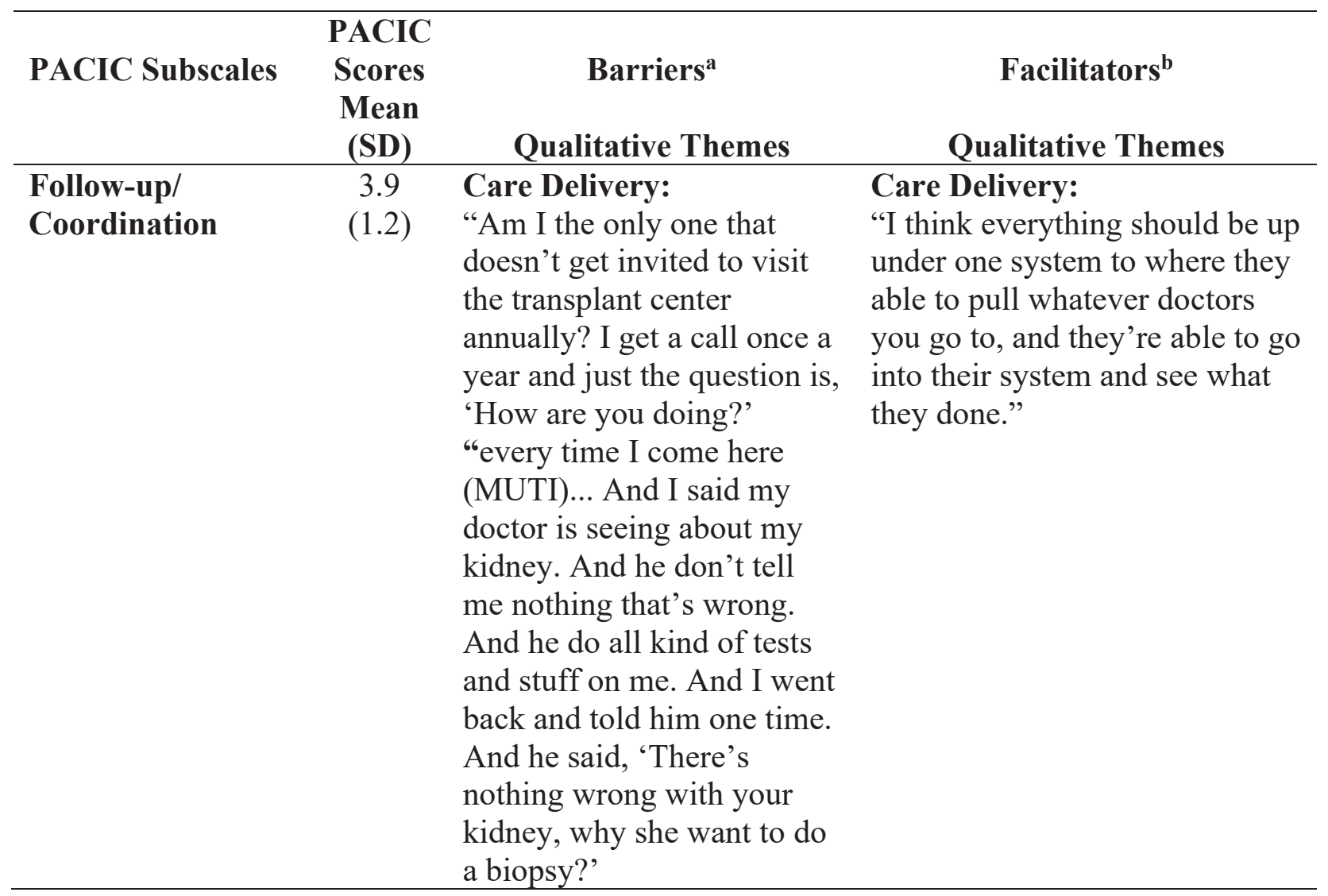

Note. $\mathrm{SD}=$ Standard deviation.

${ }^{a}$ Barriers are those themes identified by KTRs that impede the receipt of care that is congruent with the Chronic Care Model concepts.

${ }^{b}$ Facilitators are those themes identified by KTRs that promote the receipt of care that is congruent with the Chronic Care Model concepts. 
explored in sociological studies, no studies were found in the literature regarding fictive kin and its role in the patient-provider relationship.

\section{Demographic Characteristics of HCP Participant Sample}

Twenty-five nephrologists participated in the survey portion of this study, and 5 participated in individual interviews. The median age of the 25 physician participants was 55 $(\mathrm{IQR}=44-64)$ with a range of 38 to 80 years of age. Fifty-nine percent of practicing U.S. nephrologists are under the age of 55 years, while $48 \%$ of this study's participants were under the age of 55 years. The age characteristics of this sample of nephrologists ( $M d n=54.6$ years, $\mathrm{IQR}=44-64$ ) very closely mirrors those of the U.S average age of nephrologists, with $59 \%$ being under age 55. Despite the national concern of an aging medical workforce, working nephrologists are comparatively young, especially considering the average age for completion of training is 36 years. Gender characteristics of this sample also mirror the national figures.

Twenty-four percent of HCP participants were women, and $25 \%$ of practicing U.S. nephrologists are women. Caucasians made up the majority of HCPs $(15,60 \%)$, while the remainder was African American (2, 8\%) and Other (8,32\%). National data regarding practicing nephrologists' race is scant, however, according to Salsberg, Quigley, Mehfoud, Masselink, \& Collins (2016) in a study on the U.S. nephrology workforce, Asians constitute a large percentage $(43.4 \%)$ followed by Caucasians (21\%) and African Americans (6.2\%). Almost all, or $22(88 \%)$ out of the 25 physicians, possessed a nephrology board certification. The physicians had been in practice for a median of 24 years (IQR=9-30), with a range of 5 to 50 years.

\section{Practice Pattern Characteristics of HCP Participant Sample}

Most (20,80\%) of the participants reported practicing in a group as opposed to solo practice, which is greater than the reported nationwide figures of $39 \%$ of U.S. nephrologists working in group practices. The number of physicians within these group practices ranged from 2 to 30 , with a median of 5 (2-8). It is important to note that the physicians reporting 30 physicians in their group were part of an end-stage renal disease accountable care organization (ESCO) CMS pilot study serving 14 Tennessee counties. The median number of non-physician staff reported was also skewed by the ESCO 5 (1-3). (Membership in an ESCO does not change the practice patterns of CKD/transplant patients. ESCOs are strictly dialysis care providers.) The number of days physicians spend in clinic per week varies from 1 to 5 days with a median of 3 days per week available for office appointments. A median of 17 hours per week was reported as time spent with office patients (min-max 3-40). Due to the nature of nephrology practice with dialysis and hospital rounds, the report of 40 hours per week seeing office patients was likely a misunderstanding of the question. The use of electronic health records was prevalent among the HCP participants, with $21(84 \%)$ out of the 25 physicians reporting use of electronic health record in their clinics. Nationally, $75 \%$ of nephrology practices have adopted electronic health records. Almost all, or 21 (84\%) of the 25 physicians reported using evidence-based clinical guidelines in the management of KTRs. Of those who sought updates on the kidney transplant care/research, almost half $(12 ; 48 \%)$ of the physicians did so on an annual basis, with preferred methods of updates being peer-reviewed journals $(15,60 \%)$, websites $(15,60 \%)$, and 
conferences $(12 ; 48 \%)$ The HCP demographic and practice pattern characteristics are summarized in (Tables 4-15 and 4-16)

\section{HCP QUAN Results}

\section{Specific Aim Four}

The fourth aim of this study was to determine the degree to which HCPs believe the longterm care provision to their kidney transplant patients aligns with the concepts of quality care as outlined in the CCM.

Research Question 6. How do HCPs rate their overall provision of long-term care in the context of concepts identified in the CCM, as determined by the ACIC?

Research Question 7. How do HCPs rate the long-term care provision to KTRs in the context of specific concepts of the CCM, as measured by the following subscales of the ACIC?

1) organization of the healthcare system

2) community linkages

3) self-management support

4) decision support

5) delivery system design

6) clinical information system

7) integration of the CCM components into the delivery of care

The survey results from this sample of HCPs indicate that the nephrologists perceive that care provided to KTRs does not highly align with the CCM concepts of care. The subscale and summary scores for the ACIC are provided in Table 4-17 The lowest score was given in the Integration of Chronic Care Model Components with a mean of 4.41 ( \pm 2.16$)$. This subscale identifies how well the system integrates the concepts of the CCM such as linking patient goals with the organizations' information system. The next lowest scores within the ACIC domains were clinical information systems and community linkages. The highest score given by this sample of nephrologists was on the subscale Organization of the Healthcare Delivery System.

\section{Specific Aim Five}

The fifth aim of this study was to determine whether demographic and clinical practice patterns are associated with HCPs' self-reported delivery of long-term, follow-up care that aligns with the CCM concepts of quality care.

Research Question 8. Are demographic and practice patterns associated with summary and subscale scores on the ACIC in this sample of HCPs? 
Table 4-15. Demographic and practice pattern characteristics of physicians $(\mathrm{N}=\mathbf{2 5})$

\begin{tabular}{|c|c|c|}
\hline Physician Characteristic & Frequency & Percentage \\
\hline \multicolumn{3}{|l|}{ Gender } \\
\hline Male & 19 & 76 \\
\hline Female & 6 & 24 \\
\hline \multicolumn{3}{|l|}{ Race } \\
\hline Caucasian & 15 & 60 \\
\hline African American & 2 & 8 \\
\hline Other & 8 & 32 \\
\hline \multicolumn{3}{|l|}{ Nephology board certification } \\
\hline No & 3 & 12 \\
\hline Yes & 22 & 88 \\
\hline \multicolumn{3}{|l|}{ Individual or group practice } \\
\hline Individual & 5 & 20 \\
\hline Group & 20 & 80 \\
\hline \multicolumn{3}{|l|}{ Number of clinic days per week } \\
\hline 1 & 3 & 12 \\
\hline 2 & 6 & 24 \\
\hline 3 & 5 & 20 \\
\hline 4 & 7 & 28 \\
\hline 5 & 4 & 16 \\
\hline \multicolumn{3}{|l|}{ Electronic health record in clinic } \\
\hline No & 4 & 16 \\
\hline Yes & 21 & 84 \\
\hline \multicolumn{3}{|l|}{ Embedded Decision Support ${ }^{a}$} \\
\hline No & 4 & 16 \\
\hline Yes & 21 & 84 \\
\hline \multicolumn{3}{|c|}{ Frequency of kidney transplant care/research updates } \\
\hline Annually & 12 & 48 \\
\hline Once a month & 4 & 16 \\
\hline Once a week & 5 & 20 \\
\hline Rarely & 4 & 16 \\
\hline \multicolumn{3}{|c|}{ Preferred methods for kidney transplant continuing education } \\
\hline Journals & 15 & 60 \\
\hline Conferences & 12 & 48 \\
\hline Books & 4 & 16 \\
\hline Embedded Sources- Up to Date & 15 & 60 \\
\hline Email Lists & 4 & 16 \\
\hline
\end{tabular}

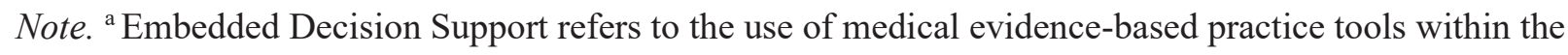
electronic medical record (i.e., UpToDate, Epocrates, Medscape). Methods for continuing education percentages exceed $100 \%$ due to selection of multiple methods of continuing education. 
Table 4-16. Descriptive statistics for summaries of HCPs continuous variables $(n=25)$

\begin{tabular}{lcccc}
\hline Statistic & Median $\left(\mathbf{2 5}^{\text {th }}-\mathbf{7 5}^{\text {th }}\right)$ & Min & Max & Mean (SD) \\
\hline Age & $55(44-64)$ & 38 & 82 & $54.64(12.19)$ \\
Years in practice & $24(9-30)$ & 5 & 50 & $22.16(12.84)$ \\
Number of physicians $^{\mathrm{a}}$ & $5(2-8)$ & 1 & 30 & $6.28(6.05)$ \\
Number of support staff $^{\text {Hours per week }}$ & $5(3-10)$ & 2 & 100 & $13.04(22.64)$ \\
& $14(6-12)$ & 3 & 40 & $17.04(11.52)$ \\
\hline
\end{tabular}

Note. $\mathrm{HCPs}=$ healthcare providers.

${ }^{a}$ Number of physicians in the practice.

${ }^{\mathrm{b}}$ Hours per week in direct patient care in clinic.

Table 4-17. Assessment of Chronic Illness Care subscale and summary scores ( $\mathrm{N}=\mathbf{2 5})$

\begin{tabular}{lcc}
\hline ACIC Dimension & Mean (SD) & Median $\left.\mathbf{( 2 5}^{\text {th }} \mathbf{- 7 5 t h}\right)$ \\
\hline Organization of Healthcare System & $6.04(2.37)$ & $6(4-7)$ \\
Community Linkages & $5.16(2.59)$ & $5(2-7)$ \\
Self-Management Support & $5.26(2.47)$ & $5(3-7)$ \\
Decision Support & $5.69(2.47)$ & $5(4-8)$ \\
Delivery System Design & $5.98(2.11)$ & $6(4-8)$ \\
Clinical Information Systems & $5.04(2.49)$ & $5(3-7)$ \\
Integration of Chronic Care Model & $4.41(2.16)$ & $4(3-6)$ \\
Total & $5.45(2.13)$ & $5(4-7)$ \\
\hline
\end{tabular}

Note. $\mathrm{SD}=$ Standard deviation. 
The analysis of associations among categorical variables and the ACIC summary and subscale scores found type of practice (i.e., solo or group) was associated with subscales SelfManagement Support $(\mathrm{p}=.02)$ and Delivery System Design $(\mathrm{p}=.05)$ as well as the summary scores $(p=.009)$ as shown in Table 4-18 to Table 4-27. Specifically, solo providers were 3 times more likely to perceive that they provide self-management support to their KTR patients that is highly aligned with the CCM concepts than those nephrologists practicing in groups. Solo providers were also 2 times more likely to perceive that they implement CCM concepts associated with delivery system design and 3 times more likely to perceive higher implementation of the overall CCM concepts of the ACIC summary score.

Associations were found between utilization of embedded decision support for kidney transplantation updates and education with Self-Management Support concepts $(p=.04)$ and Integration of the CCM components $(\mathrm{p}=.008)$. Electronic sources, such as Up to Date and Epocrates, imbedded in electronic medical records, provide current research and evidence-based practice algorithms at the point of care and offer printable patient education information. The solo providers who utilize decision support were 3 times more likely than providers who practice in a group setting to perceive higher implementation of self-management support and those who do not utilize embedded decision support were 2 times more likely to perceive that they had a low integration of the CCM concepts in their care of KTRs.

Race was significantly associated with the subscale measuring integration of the CCM concepts in care management. Only 33\% of Caucasian providers reported high levels of integration of the CCM components in their practice and AAs and those that identified as other in race were 1.8 times more likely to perceive high implementation of CCM concept integration in their care of KTRs. This is likely a spurious finding given the small number of AA HCP participants.

No associations with the ACIC subscales and provider age were found, but provider age and the summary scores were significantly associated $(\mathrm{p}=.01)$. Older physicians $(\mathrm{M}=59.9)$ perceived their care of KTRs as less aligned with the overall ACIC scale than the younger physicians $(\mathrm{M}=48)$. Further, those practitioners with more years in practice, compared to those with fewer years in practice, reported lower implementation of ACIC subscale concepts in the care of KTRs: Community Linkages ( $p=.03)$, Self-management Support $(p=.04)$, Clinical information Systems $(\mathrm{p}=.05)$, overall Integration of CCM $(\mathrm{p}=.03)$. On the summary score, younger physicians $(\mathrm{M}=48)$ reported greater alignment with the CCM than the older practitioners $(\mathrm{M}=59.9, \mathrm{p}=.007)$. There were no associations between the number of physicians in a practice and the ACIC subscale or summary scores.

\section{HCP QUAN Discussion}

Overall, this sample of HCPs do not perceive the care they provide to KTRs to be highly aligned with the concepts of the CCM. This sample of nephrologists scored the ACIC subscale Organization of the Healthcare Delivery System the highest suggesting that the participants perceive that the healthcare system is oriented to allow for focused chronic illness 
Table 4-18. Associations between healthcare provider characteristics and dichotomized Assessment of Chronic Illness Care subscale organization of healthcare delivery

\begin{tabular}{|c|c|c|c|}
\hline $\begin{array}{l}\text { Demographic } \\
\text { Characteristics }\end{array}$ & $\begin{array}{c}\text { High Implementation }^{\mathrm{b}} \mathrm{CCM} \\
\text { n (\%) }\end{array}$ & $\begin{array}{c}\text { Low Implementation }^{\mathrm{c}} \mathrm{CCM} \\
\mathrm{n}(\%)\end{array}$ & $\begin{array}{c}\mathbf{P} \\
\text { Value }\end{array}$ \\
\hline \multicolumn{3}{|l|}{ Type of Practice } & .61 \\
\hline Solo & $4(80 \%)$ & $1(20 \%)$ & \\
\hline Group & $11(55 \%)$ & $9(45 \%)$ & \\
\hline \multicolumn{3}{|l|}{ Gender } & .99 \\
\hline Male & $11(58 \%)$ & $8(42 \%)$ & \\
\hline Female & $4(27 \%)$ & $2(20 \%)$ & \\
\hline \multicolumn{3}{|l|}{ Race } & .48 \\
\hline Caucasian & $9(60 \%)$ & $6(40 \%)$ & \\
\hline $\mathrm{AA}$ & $2(100 \%)$ & $0(0 \%)$ & \\
\hline Other & $4(50 \%)$ & $4(50 \%)$ & \\
\hline \multicolumn{3}{|c|}{ Embedded Decision Support ${ }^{\mathrm{a}}$} & .99 \\
\hline Yes & $9(60 \%)$ & $6(40 \%)$ & \\
\hline No & $6(60 \%)$ & $4(40 \%)$ & \\
\hline \multicolumn{3}{|c|}{ Electronic Medical Record } & .3 \\
\hline Yes & $14(67 \%)$ & $7(33 \%)$ & \\
\hline No & $1(25 \%)$ & $3(75 \%)$ & \\
\hline
\end{tabular}

Note. $\mathrm{AA}=\mathrm{A}$ frican American, $\mathrm{HCP}=$ Healthcare provider, $\mathrm{ACIC}=\mathrm{Assessment}$ of Chronic Illness Care, $\mathrm{CCM}=$ Chronic Care Model. Due to small expected cell counts, Fisher's Exact two-tailed test was used. Numbers in parentheses indicate row percentages.

${ }^{\text {a }}$ Embedded Decision Support refers to the use of medical evidence-based practice tools within the electronic medical record (i.e., UpToDate, Epocrates, Medscape).

${ }^{\mathrm{b}}$ High implementation refers to ACIC dichotomized scores $\geq 6$.

${ }^{\mathrm{c}}$ Low implementation refers to ACIC dichotomized scores $\leq 5$. 
Table 4-19. Associations between healthcare provider characteristics and dichotomized Assessment of Chronic Illness Care subscale community linkages

\begin{tabular}{|c|c|c|c|}
\hline HCP Characteristics & $\begin{array}{c}\text { High Implementation CCM } \\
\text { n (\%) }\end{array}$ & $\begin{array}{c}\text { Low Implementation CCM } \\
\text { n (\%) }\end{array}$ & $\begin{array}{c}P \\
\text { Value } \\
\end{array}$ \\
\hline \multicolumn{3}{|l|}{ Type of Practice } & .62 \\
\hline Solo & $3(60 \%)$ & $2(40 \%)$ & \\
\hline Group & $8(40 \%)$ & $12(60 \%)$ & \\
\hline \multicolumn{3}{|l|}{ Gender } & .99 \\
\hline Male & $8(42 \%)$ & $11(58 \%)$ & \\
\hline Female & $3(50 \%)$ & $3(50 \%)$ & \\
\hline \multicolumn{3}{|l|}{ Race } & .22 \\
\hline Caucasian & $5(33 \%)$ & $10(67 \%)$ & \\
\hline $\mathrm{AA}$ & $2(100 \%)$ & $0(0 \%)$ & \\
\hline Other & $4(50 \%)$ & $4(50 \%)$ & \\
\hline \multicolumn{3}{|c|}{ Embedded Decision Support ${ }^{\mathrm{a}}$} & .4 \\
\hline Yes & $8(53 \%)$ & $7(47 \%)$ & \\
\hline No & $3(30 \%)$ & $7(70 \%)$ & \\
\hline \multicolumn{3}{|c|}{ Electronic Medical Record } & 0.1 \\
\hline Yes & $11(52 \%)$ & $10(48 \%)$ & \\
\hline No & $0(0 \%)$ & $4(100 \%)$ & \\
\hline
\end{tabular}

Note. $\mathrm{AA}=\mathrm{A}$ frican American, $\mathrm{HCP}=$ Healthcare provider, $\mathrm{ACIC}=\mathrm{Assessment}$ of Chronic Illness Care, $\mathrm{CCM}=$ Chronic Care Model. Due to small expected cell counts, Fisher's Exact two-tailed test was used. Numbers in parentheses indicate row percentages.

${ }^{\text {a }}$ Embedded Decision Support refers to the use of medical evidence-based practice tools within the electronic medical record (i.e., UpToDate, Epocrates, Medscape).

${ }^{\mathrm{b}}$ High implementation refers to ACIC dichotomized scores $\geq 6$.

${ }^{\mathrm{c}}$ Low implementation refers to ACIC dichotomized scores $\leq 5$. 
Table 4-20. Associations between healthcare provider characteristics and dichotomized Assessment of Chronic Illness Care subscale self-management support

\begin{tabular}{|c|c|c|c|}
\hline HCP Characteristics & $\begin{array}{c}\text { High Implementation CCM } \\
\text { n (\%) }\end{array}$ & $\begin{array}{c}\text { Low Implementation CCM } \\
\text { n (\%) }\end{array}$ & $\begin{array}{c}\mathbf{P} \\
\text { Value } \\
\end{array}$ \\
\hline \multicolumn{4}{|l|}{ Type of Practice } \\
\hline Solo & $5(100 \%)$ & $0(0 \%)$ & \multirow[t]{2}{*}{.02} \\
\hline Group & $7(35 \%)$ & $13(65 \%)$ & \\
\hline \multicolumn{3}{|l|}{ Gender } & \multirow[t]{3}{*}{.99} \\
\hline Male & $9(47 \%)$ & $10(53 \%)$ & \\
\hline Female & $3(50 \%)$ & $3(50 \%)$ & \\
\hline \multicolumn{3}{|l|}{ Race } & \multirow[t]{4}{*}{.15} \\
\hline Caucasian & $5(33 \%)$ & $10(67 \%)$ & \\
\hline $\mathrm{AA}$ & $2(100 \%)$ & $0(0 \%)$ & \\
\hline Other & $5(62 \%)$ & $3(38 \%)$ & \\
\hline \multicolumn{3}{|c|}{ Embedded Decision Support ${ }^{\mathrm{a}}$} & \multirow[t]{3}{*}{.04} \\
\hline Yes & $10(67 \%)$ & $5(33 \%)$ & \\
\hline No & $2(20 \%)$ & $8(80 \%)$ & \\
\hline \multicolumn{3}{|c|}{ Electronic Medical Record } & \multirow[t]{3}{*}{.6} \\
\hline Yes & $11(52 \%)$ & $10(48 \%)$ & \\
\hline No & $1(25 \%)$ & $3(75 \%)$ & \\
\hline
\end{tabular}

Note. $\mathrm{AA}=\mathrm{A}$ frican American $\mathrm{HCP}=$ Healthcare provider, $\mathrm{ACIC}=\mathrm{Assessment}$ of Chronic Illness Care, $\mathrm{CCM}=$ Chronic Care Model. Due to small expected cell counts, Fisher's Exact two-tailed test was used. Numbers in parentheses indicate row percentages.

${ }^{a}$ Embedded Decision Support refers to the use of medical evidence-based practice tools within the electronic medical record (i.e., UpToDate, Epocrates, Medscape).

${ }^{\mathrm{b}}$ High implementation refers to ACIC dichotomized scores $\geq 6$.

${ }^{\mathrm{c}}$ Low implementation refers to ACIC dichotomized scores $\leq 5$. 
Table 4-21. Associations between healthcare provider characteristics and dichotomized Assessment of Chronic Illness Care subscale decision support

\begin{tabular}{lccc}
\hline $\begin{array}{l}\text { HCP Characteristics/ } \\
\text { ACIC Association }\end{array}$ & $\begin{array}{c}\text { High Implementation CCM } \\
\text { n (\%) }\end{array}$ & $\begin{array}{c}\text { Low Implementation CCM } \\
\text { n (\%) }\end{array}$ & $\begin{array}{c}\text { P } \\
\text { Value }\end{array}$ \\
\hline $\begin{array}{l}\text { Type of Practice } \\
\text { Solo }\end{array}$ & $4(80 \%)$ & $1(20 \%)$ & .13 \\
$\quad$ Group & $7(35 \%)$ & $13(65 \%)$ & .99 \\
Gender & & & \\
$\quad$ Male & $8(42 \%)$ & $11(58 \%)$ & .99 \\
$\quad$ Female & $3(50 \%)$ & $3(50 \%)$ & \\
Race & $7(47 \%)$ & $7(53 \%)$ & .4 \\
$\quad$ Caucasian & $1(50 \%)$ & $1(50 \%)$ & \\
$\quad \begin{array}{l}\text { AA } \\
\text { Other }\end{array}$ & $3(38 \%)$ & $5(62 \%)$ & \\
Embedded Decision Support & & \\
$\quad$ Yes & $8(53 \%)$ & $7(28 \%)$ & \\
$\quad$ No & $3(30 \%)$ & $7(70 \%)$ & \\
Electronic Medical Record & $10(48 \%)$ & $11(52 \%)$ & \\
$\quad$ Yes & $1(25 \%)$ & $3(75 \%)$ & \\
No & & & \\
\hline
\end{tabular}

Note. $\mathrm{AA}=\mathrm{A}$ frican American, $\mathrm{HCP}=$ Healthcare provider, $\mathrm{ACIC}=\mathrm{Assessment}$ of Chronic Illness Care, $\mathrm{CCM}=$ Chronic Care Model. Due to small expected cell counts, Fisher's Exact two-tailed test was used. Numbers in parentheses indicate row percentages.

${ }^{\text {a }}$ Embedded Decision Support refers to the use of medical evidence-based practice tools within the electronic medical record (i.e., UpToDate, Epocrates, Medscape).

${ }^{\mathrm{b}}$ High implementation refers to ACIC dichotomized scores $\geq 6$.

${ }^{\mathrm{c}}$ Low implementation refers to ACIC dichotomized scores $\leq 5$. 
Table 4-22. Associations between healthcare provider characteristics and dichotomized Assessment of Chronic Illness Care subscale delivery system design

\begin{tabular}{|c|c|c|c|}
\hline $\begin{array}{l}\text { HCP Characteristics/ } \\
\text { ACIC Association }\end{array}$ & $\begin{array}{c}\text { High Implementation CCM } \\
\text { n (\%) }\end{array}$ & $\begin{array}{c}\text { Low Implementation CCM } \\
\text { n (\%) }\end{array}$ & $\begin{array}{c}\text { P } \\
\text { Value }\end{array}$ \\
\hline \multicolumn{3}{|l|}{ Type of Practice } & .05 \\
\hline Solo & $5(100 \%)$ & $0(0 \%)$ & \\
\hline Group & $9(45 \%)$ & $11(55 \%)$ & \\
\hline \multicolumn{3}{|l|}{ Gender } & .99 \\
\hline Male & $11(58 \%)$ & $8(42 \%)$ & \\
\hline Female & $3(50 \%)$ & $3(50 \%)$ & \\
\hline \multicolumn{3}{|l|}{ Race } & .68 \\
\hline Caucasian & $8(53 \%)$ & $7(47 \%)$ & \\
\hline $\mathrm{AA}$ & $2(100 \%)$ & $0(0 \%)$ & \\
\hline Other & $4(50 \%)$ & $4(50 \%)$ & \\
\hline \multicolumn{3}{|c|}{ Embedded Decision Support ${ }^{\mathrm{a}}$} & .24 \\
\hline Yes & $10(67 \%)$ & $5(33 \%)$ & \\
\hline No & $4(40 \%)$ & $6(60 \%)$ & \\
\hline \multicolumn{3}{|c|}{ Electronic Medical Record } & .29 \\
\hline Yes & $13(62 \%)$ & $8(38 \%)$ & \\
\hline No & $1(25 \%)$ & $3(75 \%)$ & \\
\hline
\end{tabular}

Note. $\mathrm{AA}=\mathrm{A}$ frican American, $\mathrm{HCP}=$ Healthcare provider, $\mathrm{ACIC}=\mathrm{Assessment}$ of Chronic Illness Care, $\mathrm{CCM}=$ Chronic Care Model. Due to small expected cell counts, Fisher's Exact two-tailed test was used. Numbers in parentheses indicate row percentages.

${ }^{a}$ Embedded Decision Support refers to the use of medical evidence-based practice tools within the electronic medical record (i.e., UpToDate, Epocrates, Medscape).

${ }^{\mathrm{b}}$ High implementation refers to ACIC dichotomized scores $\geq 6$.

${ }^{\mathrm{c}}$ Low implementation refers to ACIC dichotomized scores $\leq 5$. 
Table 4-23. Associations between healthcare provider characteristics and the dichotomized Assessment of Chronic Illness Care subscale clinical information systems

\begin{tabular}{lccc}
\hline $\begin{array}{l}\text { HCP Characteristics/ } \\
\text { ACIC Association }\end{array}$ & $\begin{array}{c}\text { High Implementation CCM } \\
\text { n (\%) }\end{array}$ & $\begin{array}{c}\text { Low Implementation CCM } \\
\text { n (\%) }\end{array}$ & $\begin{array}{c}\text { P } \\
\text { Value }\end{array}$ \\
\hline $\begin{array}{l}\text { Type of Practice } \\
\text { Solo }\end{array}$ & $4(80 \%)$ & $1(20 \%)$ & .13 \\
$\quad$ Group & $7(35 \%)$ & $13(65 \%)$ & \\
Gender & & & .35 \\
$\quad$ Male & $7(37 \%)$ & $12(63 \%)$ & .35 \\
$\quad$ Female & $4(67 \%)$ & $2(33 \%)$ & \\
Race & $6(40 \%)$ & $9(60 \%)$ & .4 \\
$\quad$ Caucasian & $2(100 \%)$ & $0(0 \%)$ & \\
$\quad$ AA & $3(38 \%)$ & $5(62 \%)$ & .1 \\
$\quad$ Other & & $7(47 \%)$ & \\
Embedded Decision Support & & \\
$\quad$ Yes & $8(53 \%)$ & $7(70 \%)$ & \\
$\quad$ No & $3(30 \%)$ & $10(48 \%)$ & \\
Electronic Medical Record & $11(52 \%)$ & $4(100 \%)$ & \\
$\quad$ Yes & $0(0 \%)$ & & \\
$\quad$ No & &
\end{tabular}

Note. $\mathrm{AA}=\mathrm{A}$ frican American, $\mathrm{HCP}=$ Healthcare provider, $\mathrm{ACIC}=\mathrm{Assessment}$ of Chronic Illness Care, $\mathrm{CCM}=\mathrm{Chronic}$ Care Model. Due to small expected cell counts, Fisher's Exact two-tailed test was used. Numbers in parentheses indicate row percentages.

${ }^{a}$ Embedded Decision Support refers to the use of medical evidence-based practice tools within the electronic medical record (i.e., UpToDate, Epocrates, Medscape).

${ }^{\mathrm{b}}$ High implementation refers to ACIC dichotomized scores $\geq 6$.

${ }^{\mathrm{c}}$ Low implementation refers to ACIC dichotomized scores $\leq 5$. 
Table 4-24. Associations between healthcare provider characteristics and the dichotomized Assessment of Chronic Illness Care subscale integration of CCM components

\begin{tabular}{lccc}
\hline $\begin{array}{l}\text { HCP Characteristics/ } \\
\text { ACIC Association }\end{array}$ & $\begin{array}{c}\text { High Implementation CCM } \\
\text { n (\%) }\end{array}$ & $\begin{array}{c}\text { Low Implementation CCM } \\
\text { n (\%) }\end{array}$ & $\begin{array}{c}\text { P } \\
\text { Value }\end{array}$ \\
\hline $\begin{array}{l}\text { Type of Practice } \\
\text { Solo }\end{array}$ & $3(60 \%)$ & $2(40 \%)$ & .28 \\
$\quad$ Group & $5(25 \%)$ & $15(75 \%)$ & \\
Gender & $7(37 \%)$ & $12(63 \%)$ & .6 \\
$\quad$ Male & $1(17 \%)$ & $5(83 \%)$ & .002 \\
$\quad$ Female & $1(7 \%)$ & $14(93 \%)$ & \\
Race & $2(100 \%)$ & $0(0 \%)$ & .008 \\
$\quad$ Caucasian & $5(63 \%)$ & $3(37 \%)$ & \\
AA & $8(53 \%)$ & $7(47 \%)$ & .27 \\
$\quad$ Other & $0(0 \%)$ & $10(100 \%)$ & \\
Embedded Decision Support & & \\
$\quad$ Yes & $8(38 \%)$ & $13(62 \%)$ & \\
$\quad$ No & $0(0 \%)$ & $4(100 \%)$ & \\
Electronic Medical Record & & & \\
$\quad$ Yes & & & \\
$\quad$ No & &
\end{tabular}

Note. $\mathrm{AA}=\mathrm{A}$ frican American, $\mathrm{HCP}=$ Healthcare provider, $\mathrm{ACIC}=\mathrm{Assessment}$ of $\mathrm{Chronic}$ Illness Care, $\mathrm{CCM}=$ Chronic Care Model. Due to small expected cell counts, Fisher's Exact two-tailed test was used. Numbers in parentheses indicate row percentages.

${ }^{a}$ Embedded Decision Support refers to the use of medical evidence-based practice tools within the electronic medical record (i.e., UpToDate, Epocrates, Medscape).

${ }^{\mathrm{b}}$ High implementation refers to ACIC dichotomized scores $\geq 6$.

${ }^{\mathrm{c}}$ Low implementation refers to ACIC dichotomized scores $\leq 5$. 
Table 4-25. Associations between healthcare provider characteristics and the dichotomized Assessment of Chronic Illness Care summary scores

\begin{tabular}{lccc}
\hline $\begin{array}{l}\text { HCP Characteristics/ } \\
\text { ACIC Association }\end{array}$ & $\begin{array}{c}\text { High Implementation } \\
\text { CCM n (\%) }\end{array}$ & $\begin{array}{c}\text { Low Implementation CCM } \\
\text { n (\%) }\end{array}$ & $\begin{array}{c}\text { P } \\
\text { Value }\end{array}$ \\
\hline $\begin{array}{l}\text { Type of Practice } \\
\text { Solo }\end{array}$ & $5(100 \%)$ & $0(0 \%)$ & .009 \\
$\quad$ Group & $6(30 \%)$ & $14(70 \%)$ & \\
Gender & $7(37 \%)$ & $12(63 \%)$ & .35 \\
$\quad$ Male & $4(67 \%)$ & $2(33 \%)$ & \\
$\quad$ Female & $5(33 \%)$ & $10(67 \%)$ & .22 \\
Race & $2(100 \%)$ & $0(0 \%)$ & \\
$\quad$ Caucasian & $4(50 \%)$ & $4(50 \%)$ & .09 \\
$\quad$ AA & $9(60 \%)$ & $6(40 \%)$ & \\
$\quad$ Other & $2(20 \%)$ & $8(80 \%)$ & .1 \\
Embedded Decision Support ${ }^{\mathrm{a}}$ & & & \\
$\quad$ Yes & $11(52 \%)$ & $10(48 \%)$ & \\
$\quad$ No & $0(0 \%)$ & $4(100 \%)$ & \\
Electronic Medical Record & & & \\
$\quad$ Yes & & & \\
$\quad$ No & &
\end{tabular}

Note. $\mathrm{AA}=\mathrm{A}$ frican American, $\mathrm{HCP}=$ Healthcare provider, $\mathrm{ACIC}=\mathrm{Assessment}$ of Chronic Illness Care, $\mathrm{CCM}=\mathrm{Chronic}$ Care Model. Due to small expected cell counts, Fisher's Exact two-tailed test was used. Numbers in parentheses indicate row percentages.

${ }^{\text {a }}$ Embedded Decision Support refers to the use of medical evidence-based practice tools within the electronic medical record (i.e., UpToDate, Epocrates, Medscape).

${ }^{\mathrm{b}}$ High implementation refers to ACIC dichotomized scores $\geq 6$.

${ }^{\mathrm{c}}$ Low implementation refers to ACIC dichotomized scores $\leq 5$. 
Table 4-26. Comparison of the dichotomized Assessment of Chronic Illness Care Scores with healthcare provider age and years in practice

\begin{tabular}{lcccc}
\hline $\begin{array}{l}\text { HCP Characteristics/ } \\
\text { ACIC Association }\end{array}$ & $\begin{array}{c}\text { Mean } \\
\text { High }^{\mathbf{a}}\end{array}$ & $\begin{array}{c}\text { Mean } \\
\text { Low }^{\mathbf{b}}\end{array}$ & $\mathbf{S E}^{\mathbf{c}}$ & $\begin{array}{c}\text { P } \\
\text { Value }\end{array}$ \\
\hline $\begin{array}{l}\text { Organization Healthcare } \\
\quad \text { Age }\end{array}$ & 51.6 & 59.2 & 4.83 & 0.13 \\
$\quad$ Years in Practice & 18.6 & 27.5 & 5.02 & 0.09 \\
$\begin{array}{l}\text { Community Linkages } \\
\quad \text { Age }\end{array}$ & 49.7 & 58.5 & 4.67 & .07 \\
$\quad$ Years in Practice & 16.1 & 26.9 & 4.78 & .03 \\
Self-Management Support & & & & \\
$\quad$ Age & 50.0 & 58.9 & 4.62 & .06 \\
$\quad$ Years in Practice & 16.8 & 27.1 & 4.8 & .04 \\
Decision Support & & & & \\
$\quad$ Age & 51.3 & 57.3 & 4.86 & 0.23 \\
$\quad$ Years in Practice & 18.5 & 25 & 5.11 & 0.22 \\
Delivery System Design & & & & \\
$\quad$ Age & 50.9 & 59.4 & 4.70 & .09 \\
$\quad$ Years in Practice & 18.2 & 27.2 & 4.94 & .08 \\
Clinical Inf. Systems & & & & \\
$\quad$ Age & 49.7 & 58.5 & 4.67 & .07 \\
$\quad$ Years in Practice & 16.5 & 26.6 & 4.85 & .05 \\
Integration of CCM & & & & \\
$\quad$ Age & 48.3 & 57.6 & 4.97 & .07 \\
$\quad$ Years in Practice & 14.0 & 26.0 & 5.04 & .03 \\
ACIC Summary Score & & & & \\
$\quad$ Age & 48.0 & 59.9 & 4.37 & .01 \\
$\quad$ Years in Practice & 14.7 & 28.0 & 4.50 & .007 \\
\hline$\quad$ & & & & \\
\hline
\end{tabular}

Note. Independent Sample Student's t-test was used to determine differences in high and low scores with health care provider age and year in practice.

${ }^{a}$ Mean High is the arithmetic mean of each variable with scores 6 or greater on the Assessment of Chronic Illness Care on the corresponding subscale or summary score.

${ }^{\mathrm{b}}$ Mean High is the arithmetic mean of each variable with scores less than 6 on the Assessment of Chronic Illness Care on the corresponding subscale or summary score. 
Table 4-27. ACIC subscale and summary scores $(\mathbf{N}=\mathbf{2 5})$

\begin{tabular}{|c|c|c|c|}
\hline ACIC Dimension & $\begin{array}{c}\text { Mean } \\
(\mathrm{SD})\end{array}$ & Barriers & Facilitators \\
\hline $\begin{array}{l}\text { Organization of } \\
\text { Healthcare System: } \\
\text { Prioritization of chronic } \\
\text { illness management through } \\
\text { healthcare system design, } \\
\text { leadership, and policies }\end{array}$ & $\begin{array}{c}6.04 \\
(2.37)\end{array}$ & $\begin{array}{l}\text { Practice Limitations: } \\
\text { "I am not complaining about my } \\
\text { income, but most nephrologists" } \\
\text { income is derived from dialysis- } \\
\text { dialysis patients and medical } \\
\text { directors' fees, and, of course, } \\
\text { hospital consults. The time in } \\
\text { clinic seeing CKD patients, } \\
\text { including transplant patients, is a } \\
\text { small part of my practice and } \\
\text { reimbursement, but it's a big } \\
\text { expense to keep the doors open." }\end{array}$ & None reported. \\
\hline $\begin{array}{l}\text { Community Linkages: } \\
\text { Mobilizing community } \\
\text { resources to meet the needs } \\
\text { of consumers and encourage } \\
\text { consumer participation }\end{array}$ & $\begin{array}{c}5.16 \\
(2.59)\end{array}$ & $\begin{array}{l}\text { Knowledge: } \\
\text { "It would be nice to have a list of } \\
\text { resources available-I try to give } \\
\text { nutritional information and such, } \\
\text { but I nor my staff have the time to } \\
\text { search down everything. I think } \\
\text { the transplant coordinator/social } \\
\text { work could take on that role." }\end{array}$ & None reported. \\
\hline \multirow[t]{2}{*}{$\begin{array}{l}\text { Self-Management } \\
\text { Support: } \\
\text { Strategies that include } \\
\text { assessment, goal-setting, } \\
\text { action-planning, problem- } \\
\text { solving, and follow-up and } \\
\text { emphasizes the person's } \\
\text { central role in their health }\end{array}$} & $\begin{array}{c}5.26 \\
(2.47)\end{array}$ & $\begin{array}{l}\text { Health Literacy: } \\
\text { "I believe that there is evidence } \\
\text { of an association with health } \\
\text { literacy and transplant } \\
\text { outcomes - in this community, } \\
\text { we have very low health literacy, } \\
\text { and that contributes to problems } \\
\text { with providing appropriate } \\
\text { education to the patient }\end{array}$ & $\begin{array}{l}\text { Patient } \\
\text { Engagement: } \\
\text { "I have some patients } \\
\text { who are very } \\
\text { knowledgeable and } \\
\text { conscientious, no one } \\
\text { wants to go back on } \\
\text { dialysis,.." }\end{array}$ \\
\hline & & $\begin{array}{l}\text { "Compliance is a challenge. The } \\
\text { list of unpleasant side-effects } \\
\text { from all of the required } \\
\text { medications is long-and patients } \\
\text { often opt to skip them on } \\
\text { occasion, despite knowing the } \\
\text { importance of those to } \\
\text { maintaining their graft." }\end{array}$ & $\begin{array}{l}\text { "We could probably } \\
\text { do a better job of } \\
\text { educating the patient } \\
\text { before referring them } \\
\text { to transplant, and } \\
\text { transplant centers } \\
\text { could continue to } \\
\text { reinforce the } \\
\text { education." }\end{array}$ \\
\hline
\end{tabular}


Table 4-27. (Continued)

\begin{tabular}{|c|c|c|c|}
\hline ACIC Dimension & $\begin{array}{l}\text { Mean } \\
\text { (SD) }\end{array}$ & Barriers & Facilitators \\
\hline $\begin{array}{l}\text { Decision Support: } \\
\text { Promotion of clinical care } \\
\text { that is consistent with } \\
\text { scientific evidence and } \\
\text { consumer preferences }\end{array}$ & $\begin{array}{c}5.69 \\
(2.47)\end{array}$ & $\begin{array}{l}\text { Knowledge: } \\
\text { "Managing the risk of toxicity } \\
\text { and rejection related to } \\
\text { immunosuppressive therapy is } \\
\text { always a topic of interest. I'd be } \\
\text { interested in learning more about } \\
\text { steroid-free protocols." }\end{array}$ & $\begin{array}{l}\text { Communication: } \\
\text { "There should be } \\
\text { some standardized } \\
\text { process of } \\
\text { transitioning patients } \\
\text { from the care of the } \\
\text { transplant centers } \\
\text { back to their } \\
\text { nephrologist. We } \\
\text { should receive } \\
\text { clinical updates." }\end{array}$ \\
\hline $\begin{array}{l}\text { Delivery System Design: } \\
\text { transforming a system that } \\
\text { is essentially reactive-- } \\
\text { responding mainly when a } \\
\text { person is sick-to one that } \\
\text { is proactive and focused on } \\
\text { keeping a person as healthy } \\
\text { as possible }\end{array}$ & $\begin{array}{c}5.98 \\
(2.11)\end{array}$ & $\begin{array}{l}\text { Communication/Coordination: } \\
\text { "Another big problem is } \\
\text { communication with the } \\
\text { hospitals. If a patient is admitted } \\
\text { to a hospital where I maintain } \\
\text { privileges, it's a little easier, but I } \\
\text { often am not notified that my } \\
\text { patient was hospitalized." }\end{array}$ & $\begin{array}{l}\text { Access to Care: } \\
\text { "I have a few } \\
\text { transplant patients } \\
\text { who live a few hours } \\
\text { away. It is hard for } \\
\text { them to keep their } \\
\text { appointments - they } \\
\text { usually try to plan } \\
\text { all of their medical } \\
\text { visits in } 1 \text { day-if } \\
\text { one clinic is running } \\
\text { behind, it can throw } \\
\text { off the others. If } \\
\text { they have a primary } \\
\text { in their area, I work } \\
\text { with them ordering } \\
\text { labs, or send them to } \\
\text { a nearby hospital } \\
\text { out-patient lab and } \\
\text { monitor them the } \\
\text { best I can until they } \\
\text { can come in." }\end{array}$ \\
\hline
\end{tabular}


Table 4-27. (Continued)

\begin{tabular}{|c|c|c|c|}
\hline ACIC Dimension & $\begin{array}{c}\text { Mean } \\
\text { (SD) }\end{array}$ & Barriers & Facilitators \\
\hline $\begin{array}{l}\text { Clinical Information } \\
\text { Systems: } \\
\text { Organization of patient and } \\
\text { population data to facilitate } \\
\text { efficient and effective care }\end{array}$ & $\begin{array}{c}5.04 \\
(2.49)\end{array}$ & $\begin{array}{l}\text { "We were all sold on or really } \\
\text { forced into adopting expensive } \\
\text { electronic health record software } \\
\text { systems. If you want to avoid } \\
\text { payment penalties, you had to-it } \\
\text { was going to be the panacea-it } \\
\text { would fix all of the problems we } \\
\text { have with coordinating care. Well, } \\
\text { it isn't the panacea because no one } \\
\text { has access to all systems." }\end{array}$ & None reported. \\
\hline $\begin{array}{l}\text { Integration of Chronic } \\
\text { Care Model: } \\
\text { Integration of elements of the } \\
\text { Chronic Care Model }\end{array}$ & $\begin{array}{c}4.41 \\
(2.16)\end{array}$ & $\begin{array}{l}\text { Practice Limitations: } \\
\text { "Most of these patients are trying } \\
\text { to scrape together the money for } \\
\text { the medications that they have to } \\
\text { have, I don't think they'd like to } \\
\text { be billed now for something that I } \\
\text { have done for years-free of } \\
\text { charge to them." }\end{array}$ & $\begin{array}{l}\text { "I've always } \\
\text { provided those } \\
\text { services-all of } \\
\text { my patients have } \\
\text { a chronic } \\
\text { condition- } \\
\text { many chronic } \\
\text { conditions, in } \\
\text { fact. If you are } \\
\text { taking care of } \\
\text { your patients, } \\
\text { you spend time } \\
\text { on the phone } \\
\text { with them } \\
\text { reviewing labs } \\
\text { or an acute } \\
\text { concern." }\end{array}$ \\
\hline
\end{tabular}


management through leadership support. Wagner et al. (2002) identified leadership support of change and chronic illness management as a crucial element in realizing sustainable change in healthcare quality improvements. This is an important factor in developing a sustainable model of care for people with all stages of kidney disease. Interestingly, despite the perception that the healthcare system is somewhat oriented to allow for quality chronic illness management, the providers perceive low levels of integration of CCM concepts in their practices with the lowest score assigned to Integration of CCM components. The next lowest score on the ACIC subscales was Clinical Information Systems. While the participants in the original assessment of the ACIC (Bonomi et al., 2002) scored Clinical Information Systems the lowest subscale as well, it is somewhat surprising that several years after the implementation of the Health Information Technology for Economic and Clinical Health (HITECH) Act and meaningful use of electronic health records, that inadequate information systems remain an obstacle in chronic illness management in this sample. Nationally, almost three-quarters of U.S. outpatient offices were utilizing EMRs, but just over one-quarter planned to apply for meaningful use incentive payments. In fact, less than half of those with EMRs reported that their systems were fully functional. The almost 100\% reported use of EMRs in this sample and the low scores in clinical information systems supports that many systems are inadequate for the demands of modern medical practice.

Dialysis remains the greatest source of revenue for nephrologists in the U.S., likely explaining that there is little published data on specific practice patterns related to the management of CKD including KTRs. This study helps address this gap in research highlighting areas of difficulty with provision of care for KTRs that is consistent with the CCM. Speratti et al. (2019) found insufficient time as a barrier to providing care that aligns with CCM in complex diseases. The HCP participants identified limited time available for clinic visits, reporting a median of 3 days and 14 hours per week with office patients. A recent study investigating the length of time for U.S. patients to obtain appointments with specialists indicates that the average wait for a nephrology appointment is 23.5 days (athenahealth.com). Long appointment wait times are exacerbated by the frequency of patient cancellations which reach $25 \%$ with wait times reaching 4 weeks. Given that a large contributor to a nephrology practice's cash flow stems from dialysis, hospital consults, and medical directorships, it could be inferred that less time is available to spend in preventive-type care.

Interestingly, solo practices were associated with higher scores on many of the ACIC subscales and summary scores. This finding appears counterintuitive as one might hypothesize that more practitioners would afford more time with complex patients and thereby more alignment with the CCM. The solo practice association with higher implementation of many of the CCM components deserves more consideration in light of the trend of practice mergers and hospital buyouts which purport to improve access to quality integrated care.. Overall, these findings appear to align with research that indicates smaller and solo practices have better patient outcomes and lower hospital admission rates (Kralewski, Dowd, \& Xu, 2012).

Further, the utilization of embedded decision support within the electronic medical records (EMRs) was associated with higher scores in Self-Management support, Integration of CCM Components, and the summary score. Considering that EMR is a cornerstone of the CCM, it is an important finding that embedded decision support was associated with higher scores of 
these scales. The importance of technology to the improvement of chronic illness management in the CCM might partially explain that the older HCPs in this study perceived lower implementation of the CCM concepts with lower scores on all ACIC subscales and significantly lower than younger providers on the summary score. These results might be explained by prior reports that younger physicians are more comfortable with EMR and in fact have suggested that EMR might lead to early retirement, partially because of the belief by some physicians that these systems are meant to limit autonomy and nullify their medical expertise (Ajami \& Tadi, 2013; Ballard et al., 2016; Khanart, Marc, Crosby, \& Sansousi, 2018; Slade, 2016).

\section{HCP QUAL Results}

\section{Specific Aim Six}

The sixth aim of this study was to develop a rich understanding of the HCPs' experiences with provision of long-term chronic illness care for KTRs, including experiences with the larger healthcare system, access to care, coordination of care, relationships with patients, and personal practice implications.

Research Question 9. How do the HCPs experience the care of transplant recipients?

Research Question 10. What barriers and facilitators to the provision of quality long-term care are experienced?

Individual interviews were conducted with HCPs. The semi-structured discussion questions were meant to elicit a more complete understanding of the experiences of nephrologists providing long-term follow-up care for KTRs. The participants also discussed the perceived barriers and facilitators to follow-up care that is congruent with the CCM concepts. The questions that guided the discussion are provided in Appendix G.

All participants stated that management of KTRs is complex and believed more standardization of the transition of patients from the transplant centers to the community provider would be helpful. The emerging themes that the researcher identified fell under several categories. Under the category of system/provider barriers, the themes were: (a) communicationcoordination, (b) practice limitations, and (c) knowledge. Under patient barriers, the themes included: (a) health literacy and (b) adherence. Under the category of system/provider facilitators, the theme was access to care or a provider relationship. Under the category of patient facilitators, the theme was patient engagement.

\section{Barriers - System/Provider}

\section{Communication-Coordination}

The HCPs identified numerous issues related to the lack of communication and coordination of care with KTRs. Especially troublesome was the initial transfer of care from 
transplant centers. One nephrologist stated, "I have patients who have gotten their transplant at a variety of centers - Vanderbilt, UAB, Methodist - the communication from these centers after the patient has received the transplant varies. When they do communicate or provide an update about the patient's transplant, it is often inadequate." Another participant supported this sentiment, stating, "There should be some standardized process of transitioning patients from the care of the transplant centers back to their nephrologist. We should receive clinical updates."

The communication-coordination gap does not rest solely with the transplant centers. The interviewees identified problems with coordinating with other providers and hospitals, with one explaining, "These patients often have many co-morbidities and they see a lot of physicians or other practitioners - they are sometimes unaware of the drug interactions with immune suppression medications. The patient doesn't know - that's just one example of problems with communication between doctors seeing kidney transplant patients." This nephrologist added, "Another big problem is communication with the hospitals. If a patient is admitted to a hospital where I maintain privileges, it's a little easier, but I often am not notified that my patient was hospitalized." Another participant stated, "We were all sold on or really forced into adopting expensive electronic health record software systems. If you want to avoid payment penalties, you had to - it was going to be the panacea - it would fix all of the problems we have with coordinating care. Well, it isn't the panacea because no one has access to all systems."

\section{Practice Limitations}

Participants identified several practice limitations that influence the care of KTRs. Several participants noted that the largest revenue stream in nephrology is related to dialysis patients and medical directorships for dialysis units. One participant cited, "I am not complaining about my income, but most nephrologists' income is derived from dialysis - dialysis patients and medical directors' fees, and, of course, hospital consults. The time in clinic seeing CKD patients, including transplant patients, is a small part of my practice and reimbursement, but it's a big expense to keep the doors open." Another stated, "The kind of [phone] messages I would ask my MA[medical assistant] to do would have been messages that were capable of being handled by medical assistants. But, the requirements for the code were that it had to be licensed staff. So, we had to use licensed staff to meet the requirement."

When asked specifically about incorporating the CCM codes into their practice, the response was unanimous amongst the interview participants that they have always provided that care for their patients and the reimbursement does not equate to the time and money it takes to document the care and bill for it. One cited, "I've always provided those services - all of my patients have a chronic condition - many chronic conditions, in fact. If you are taking care of your patients, you spend time on the phone with them reviewing labs or an acute concern." Another had actually considered adding the CCM billing but determined that it did not make sense fiscally, explaining, "You get paid about \$30 a month per patient. You have to document the 20-minute interaction with the patient, and that takes more than 20 minutes and then the cost of billing."

One HCP pointed out that his patients would not be happy to receive a bill or a copay, explaining, "Most of these patients are trying to scrape together the money for the medications 
that they have to have, I don't think they'd like to be billed now for something that I have done for years - free of charge to them."

\section{Knowledge}

Many interview participants acknowledged that they have little opportunity to stay up-todate on the latest transplant research and management and that they could benefit from a more established relationship with the transplant centers for education on new protocols. Examples of opportunities for education most often centered on immunosuppressive therapies and other protocols such as biopsy. Participants explained, "Managing the risk of toxicity and rejection related to immunosuppressive therapy is always a topic of interest. I'd be interested in learning more about steroid-free protocols," followed by, "What are the results or impact on management with the changes to the organ allocation system?" and "Are there any advancements in personalized immune therapy?"

Another participant perceived that there should be more information available about the resources for transplant patients, stating, "It would be nice to have a list of resources available-I try to give nutritional information and such, but I nor my staff have the time to search down everything. I think the transplant coordinator/social work could take on that role."

\section{Barriers - Patients}

\section{Health Literacy of the Patients}

The HCPs identified low levels of health literacy in the population as a barrier to effective care. "I believe that there is evidence of an association with health literacy and transplant outcomes - in this community, we have very low health literacy, and that contributes to problems with providing appropriate education to the patient." Another participant hypothesized that the change of access to information might contribute, stating, "These patients were used to having interactions with a doctor or nurse several times a week - if they had a question, someone was there to answer it - now they have a bunch of new medicines and concerns but are not in contact like they were. We could probably do a better job of educating the patient before referring them to transplant, and transplant centers could continue to reinforce the education."

\section{Patient Adherence}

All of the participants identified patients' adherence to medication and follow-up regimen as a problem. The participants acknowledged that non-adherence is associated with a number of factors, including health literacy, as well as financial issues such as medication costs, copays for medical care, job loss, and insurance coverage. One physician noted, "I read somewhere that in Tennessee, over $30 \%$ of people on Medicare are living on social security alone. That number is probably higher in this area and considerably more in ESRD." Another commented that patients 
often skip their lab draws and appointments, explaining, "When people are feeling well, they will miss their follow-up appointments and labs, and then, of course, there is the financial issue." Another participant cited, "Even though a financial review is part of the pre-transplant listing process, the fact that Medicare coverage ends after 3 years is a big problem for the majority of transplant patients."

The participants identified the side effects of medications as a major contributor to nonadherence to medication regimen, with one explaining, "Compliance is a challenge. The list of unpleasant side-effects from all of the required medications is long - and patients often opt to skip them on occasion, despite knowing the importance of those to maintaining their graft."

\section{Facilitators - System/Provider}

\section{Access to Care}

The interviewees identified access to care and services as a primary system/provider facilitator. For instance, one participant noted, "My patients who live close to the Memphis metro area are at an advantage because of access to care - the transplant center, city services like transportation are important to adhering to the necessary regimen."

\section{Provider Relationship}

All participants confirmed that it is more difficult to provide follow-up care to those who live in rural areas, but a close relationship helps. This sentiment was exemplified by one HCP's comment that "I have a few transplant patients who live a few hours away. It is hard for them to keep their appointments - they usually try to plan all of their medical visits in 1 day - if one clinic is running behind, it can throw off the others. If they have a primary in their area, I work with them ordering labs, or send them to a nearby hospital out-patient lab and monitor them the best I can until they can come in." Another physician pointed to the importance of the patientprovider relationship in access to care, stating, "All of my transplant patients have been my patient since they were first diagnosed with CKD, so we have a long and I think good relationship. They know my staff and some know my family. I think that relationship is important - they know who to call if they have any problems."

\section{Facilitators - Patients}

The HCPs reported that a patient who is knowledgeable about their health and actively involved in their care was the most important patient facilitator to good outcomes. Overall, the HCPs believe that their patient population needs more education about their chronic conditions and about the kidney transplant. Providers pointed out that those who are knowledgeable and adherent are easier patients with fewer hospitalizations. One participant expressed, "I have some patients who are very knowledgeable and conscientious, no one wants to go back on dialysis, but 
some people just don't seem to put the pieces together." Another provider pointed out transplant patients who are educated about kidney disease and are actively engaged are not only going to experience better outcomes but noted that "they can play important roles in their families and communities in prevention of kidney disease."

\section{HCP QUAL Discussion}

The QUAL strand of the HCP study sample sought to describe the experience of HCPs in the management of KTRs and identify the barriers and facilitators to receiving effective longterm, follow-up care. There are few studies on nephrology practice patterns outside of dialysis in the U.S. However, as our country's healthcare system moves from a fee-based model to quality models, these providers were well aware of the importance of quality assurance and improvement efforts, as well as the influence of patient-reported outcomes, to the development of new healthcare reimbursement models. This knowledge, coupled with the completion of the ACIC survey prior to the interview, might have contributed to the HCPs' segregation of barriers and facilitators to system/provider and patient.

The HCPs highlighted continued problems with coordination of care despite the fact that the majority of HCPs have EMRs and remote access to hospitalized patients' data. The nephrologists noted that often they are not notified of hospital admissions at hospitals where they do not have privileges. It was also noted that often one receives too much information when volumes of inconsequential information are scanned into the patient record.

Furthermore, knowledge/health literacy were discussed as barriers to effective care management for both providers and patients. Health literacy and adherence are recognized by the HCPs as going hand in hand. Many patients do not keep appointments if they are feeling well, and often the patients do not comprehend the importance of adherence to the entire regimen. The HCP also acknowledged that financial constraints are often a contributing factor.

The HCPs admitted that there is little time in a general nephrology clinic to keep up with all of the transplant-specific research. It was noted that a close relationship with the transplant center is important in co-management.

The interview participants recognized two major facilitators to quality care management as access to a nephrologist with whom a patient has a good relationship and patient interest or engagement in their own health outcomes.

\section{HCP QUAN + QUAL Integration}

The ACIC survey was designed as a practical tool to aid healthcare quality improvement based on six areas which influence the organization of care. This tool is quite different from those quality measures that assess outcomes, productivity, or specific processes. The ACIC results indicated that chronic illness management is a priority, with the highest scores being found in the Organization of Healthcare domain. This is not surprising, as our nation's healthcare 
system seeks to change from the episodic acute care model to one focused on planned care for disease prevention and chronic illness care. However, in individual interviews, the HCPs noted that there are barriers to care that stem from the varied processes of transitioning transplant recipients back to their long-term management. Further research is needed in the varying transition of care processes among U.S. transplant center. It is possible that the transition process or lack thereof, is a contributor to "center effect" and varying regional outcomes for KTRs.

The ACIC results indicate that the HCPs do not perceive that KTR care is highly aligned with the concepts of the CCM. The low scores in the Clinical Information Systems domain which refers to the organization of patient and population data utilizing disease registries and reminders were supported by the interview findings. Individual interviews revealed that, despite implementation of EMR, they are not fulfilling the needs of the provider or the patients. The problems identified with EMR were the inability to access all of the patients' information from hospitalizations and other providers' office visits to prescribed medications. In addition, the providers believe that the use of EMRs increases their workloads- a problem for those who identify time limitations as a barrier to care. All of these factors have a detrimental impact on communication and coordination of care and influence the providers' perception of their ability to provide care that is congruent with the concepts of the CCM.

Overall, the scores on the ACIC were indicative of lower implementation of all of the CCM components. The qualitative findings supported the survey findings indicating low implementation of the elements of care management within this domain. Lastly, the HCPs identified weaknesses in the Community Linkages domain. Community linkages remains one of the most challenging aspects of the CCM to implement and subsequently is the least studied. However, as the healthcare system increasingly focuses on patient and community engagement this domain requires serious consideration. In fact, in a recent survey of U.S. ambulatory care it was found that clinicians do not employ a R.N. or social worker, staff whose roles often include education and links to community services (Donelan et al., 2019). These findings were also echoed in the interviews whereby physicians noted that they do not have the support of social work and dieticians in their chronic clinics and believe support-perhaps from the transplant center social workers-would be beneficial in improving the connection of patients to community resources.

The HCPs also identified low health literacy coupled with financial hardships amongst their patients as a barrier to providing care that is congruent with the CCM. While providers recognized that employment of RNs and social workers would likely improve care coordination, alleviate time constraints, improve patient education and connection to community resources they also acknowledged there are significant practice related financial barriers which inhibit the addition of such staff.

The complexity of healthcare and the seemingly failed promises of technology, thus far, have increased provider and patient burden. These increased burdens and methods to alleviate patient and provider burdens in the frequently changing U.S. healthcare system require more study as we grapple with physician burnout and patients who are already managing multiple complex conditions. 


\section{CHAPTER 5. DISCUSSION}

The following chapter will provide the integration of the results of the KTR and HCP convergent parallel studies examining perceptions of chronic illness management following kidney transplantation. The premise of the globally accepted CCM that has framed much of the healthcare policy changes in the U.S. provided the framework for this study. The CCM maintains that organizations that employ the six constructs of the model will result in knowledgeable, proactive providers and patients, thereby improving chronic care management and ultimately patient outcomes. The following chapter provides a discussion of the integration of the findings of the KTR and HCP studies.

\section{KTR and HCP QUAN Integration}

The results of the KTR and HCP surveys were highly divergent. The KTRs reported care that is almost always or always congruent with the concepts of the CCM in each of the PACIC subscales, while the HCPs perceived providing care congruent with the CCM as a basic level of implementation on all ACIC subscales except Organization of the Healthcare System, which they rated reasonably good.

After the scores from the two participant samples were recoded into categories that indicate if long-term, follow-up care of KTRs is either low or high in alignment with the CCM concepts. Eighty-one percent of patients perceived their care was highly aligned with the CCM concepts, while only $44 \%$ of HCPs perceived the care provided was highly aligned with the CCM concepts $(14.22, p=.0002)$. As has been previously discussed, the PACIC and the ACIC do not align in the measurement of the $6 \mathrm{CCM}$ concepts, as it was believed that patients would have limited knowledge of the organization of the healthcare system and clinical information systems.

Of the CCM concepts assessed by both surveys the KTRs and HCPs assigned the highest scores to Delivery System Design- actions that organize care. This is interesting considering that some of the items that make up this concept include follow-up and coordination on the ACIC, but questions related to follow-up and coordination on the PACIC are assessed in a separate subscale. Notably, the KTRs gave the lowest scores to Follow-up/Coordination, and HCPs gave the lowest scores to Integration of the CCM Concepts.

Although the PACIC and ACIC surveys were designed to assess patient and provider assessments- which included other non- medical staff- of the implementation of care that aligns with the CCM, the discordance in the conceptualization of the domains and the scoring make a direct comparison of the results of the two surveys complex, particularly with small sample sizes.

The researcher found only one other study that utilized both the PACIC and the ACIC in 39 primary healthcare facilities with over 1700 patient and 283 practice participants nested within the practices. (Noel et al., 2014). The findings of this study also highlight the uniqueness

of the subscales of the two surveys. 


\section{KTR and HCP QUAL Integration}

The qualitative integration of the KTRs and HCPs provided more congruency in perceptions of chronic illness management and the barriers and facilitators between HCPs and KTRs, Overall, focus group results were positive with regard to the care received from their nephrologist; however, the KTRs did express concerns with receiving adequate transplant education (Self-Management Support), however, they believe overwhelmingly that their nephrologist provided education. The HCPs acknowledged low health literacy amongst their KTR patients and transplant specific education amongst themselves as barriers to care.

Further, focus group findings align with the HCP interview results in elucidating gaps in coordination of care. Both participant groups discussed insurance and patient financial concerns as barriers to care, while the patients did not identify any provider practice limitations, such as staffing and time, which were identified by the HCP. The KTRs did note that lack of connection to community resources that were more readily available in the dialysis setting, i.e., social workers and dieticians. One note of divergence in the barriers to care that was perceived by the KTRs but was not identified by HCPs was related to mental/emotional health. Both participant groups were clear that a major concern for KTRs is avoidance of a return to dialysis. Importantly, KTRs and HCPs discussed the importance of the long relationship with one another and patient engagement as facilitators in receiving high-quality care. Table 5-1 contains the summary of the themes from the two groups.

\section{Discussion}

This study is the first to explore KTRs and HCPs perceptions of chronic care management that aligns with the concepts of the CCM. Other studies in patient populations with chronic conditions such as diabetes, hypertension, and congestive heart failure, all of which are prevalent conditions in KTRs have shown elements of the CCM to effectively inform caremanagement (McCoy et al., 2015; Noel et al., 2016; Sendell et al., 2017). The economic and social burden of CKD have been identified as a global concern. U.S. policy has recently identified the importance of kidney transplant with a focus on increasing transplantation as the preferred renal replacement therapy and issued a call for proposals of new models of care. As the CCM has provided the framework for ongoing reform of the U.S. healthcare system, the findings of this study provide an important foundation for future studies in quality chronic care management in this vulnerable population. Interestingly, outcomes research in CKD and ESRD has often excluded the KTR population and their HCPs. The patient and provider are central to effective, efficient healthcare in the CCM framework. This study brings the voice of these key stakeholders to the conversation as researchers and policy makers seek answers to find new models for kidney care.

There were several unique findings in this study. One important finding was the discrepancy between the KTRs and HCPs quantified perceptions of care aligning with the CCM concepts. This is particularly important as the role of survey patient reported outcomes and satisfaction increasingly inform reimbursement models. In this study, the KTRs reported their care more positively than the HCPs. 
Table 5-1. Comparison of the qualitative results from the focus group and interviews

\begin{tabular}{|c|c|c|}
\hline Factors & Focus Group (KTR) & Interview (HCP) \\
\hline \multirow[t]{5}{*}{ Barriers } & Lack of knowledge & Patient health literacy \\
\hline & Care delivery & Adherence \\
\hline & Community resources & $\begin{array}{l}\text { Poor communication- } \\
\text { coordination }\end{array}$ \\
\hline & Mental/emotional health & Practice limitations \\
\hline & & Provider transplant knowledge \\
\hline \multirow[t]{3}{*}{ Facilitators } & Support & Access to care \\
\hline & Self-engagement & Provider relationship \\
\hline & Provider relationship & Patient engagement \\
\hline
\end{tabular}


However, care coordination and follow-up remain central barriers to chronic illness management. This sample of KTRs also identified transplant education as a barrier to care. A systematic review of kidney transplant education interventions (Kristin et al., 2012) found that there was limited evidence of positive education outcomes. Patient education is foundational to patient engagement and is critical in this vulnerable population that has been identified as having low health literacy and efficacy. Efforts to individualize patient education utilizing modalities that are effective for a particular patient are necessary to promote KTR patient engagement.

Even though KTR focus groups identified numerous barriers to long-term care management, the nephrologist was overwhelmingly not held accountable for those shortcomings. Alternatively, the HCPs believed that KTR care management could be improved and took responsibility for problems with care coordination and limited kidney transplant specific knowledge. Successful CCM implementation is predicated on integrated information systems. With the ever-increasing rate with which medical and pharmaceutical knowledge is produced, the importance of providers identifying lack of readily accessible, accurate information must not be overlooked.

A recent study by Sperati et al. (2019) found that a major barrier for primary care physicians' management of CKD patients is the complexity of comorbid conditions coupled with a lack of useful algorithms of care- noting a lack of straightforward guidelines like those found for diabetes management. This sentiment was echoed by the HCPs in this study stating that decision support tools are not as useful in complex KTR care. Noting that more standardized information and more formal and intentional communication with the transitioning transplant center could minimize risks associated with transitions of care. Although such development efforts were beyond the scope of this study, at a minimum, such a plan should include the pretransplant evaluation, medications, transplant clinic notes, current laboratory assessments, planned annual transplant center evaluation, and social work assessment, including Medicare/Medicaid status and drug coverage.

The only HCP demographics that were associated with the perception of CCM implementation were age and years in practice. Older nephrologists, with more years in practice, perceive lower implementation of CCM. No other studies were identified associating years in practice and age with lower implementation of CCM concepts. This finding is particularly important in nephrology as the specialty grapples with lagging interest among medical students and residents. Another unique finding is the association of solo practitioners' greater perception of CCM concept implementation. This finding might be a factor of solo practices having fewer patients overall and therefore more able to manage the chronic care needs of their KTR patients. Alternatively, this finding could be a function of autonomy empowering the providers to implement care without the bottom line focus which is often associated with larger groups.

In this study, the HCPs voiced a willingness to improve chronic illness management, but identified limited time and practice financial constraints as barriers. The introduction of new technologies such as telehealth and monitoring devices are all exciting propositions, but these technologies increase staffing needs to appropriately manage the influx of information. Any successful model of kidney patient chronic care management will need to include reimbursement reform. 
Additionally, this study contributes to the body of research identifying the importance of the patient provider relationship. Past studies have identified a good patient- provider relationship with improved communication and therefore improved outcomes. In this study, the KTRs identified strongly with the provider as a part of their family. The fictive kin relationship has been studied in multiple settings worldwide, but not in healthcare. As we look at new models of care implementing many technological benefits, the importance of the patient- provider relationship to outcomes deserves close study.

The patient and provider identified barriers to CCM were considered, and possible examples of system implementation are described in Table 5-2. These goals and examples of implementation parallel many of those already utilized in the care of patients receiving dialysis. One consideration to improve access and quality of KTRs care while reducing costs is to utilize the extensive network of multidisciplinary care already in place in dialysis centers throughout the country.

ESRD is funded predominantly through public insurance; therefore, this could provide for an easier adoption of seamless care payment models. An additional benefit to this adaptation is that the majority of KTRs are familiar with the setting, their nephrologist is seeing patients there weekly, and they are accustomed to the integrated multidisciplinary care, i.e., nephrologists, nurse practitioners, dieticians, social workers, and pharmacists.

\section{Limitations}

There were several limitations. First, despite the intended use of these surveys in this study, to this researcher's knowledge, only one set of researchers have previously utilized both instruments to determine the alignment of patient and provider perceptions of CCM implementation in a primary care setting (Noel et al., 2016). The current investigation is the only study to utilize these instruments in the assessment of care management following kidney transplantation.

Second, the researcher recruited a small sample, which limited the researcher's ability to nest the KTRs within the HCP practice and capacity for statistical analyses. The small sample size decreased the statistical power of the study; therefore, the results may not be completely representative of the population that was examined. Moreover, it may not be possible to generalize the results of the quantitative components of the study to all kidney transplant patients in the United States due to the small size and non-representativeness of the sample.

Third, the findings are limited by the cross-sectional nature of the analysis, the restriction to a narrow geographical region of the United States, and the researcher's selection bias inherent in the convenience samples of HCPs utilized for the study. It is possible that there was selection bias in the KTR participants in that the researcher excluded hospitalized patient participation. In addition, the focus group participants were limited to those who could travel to MUTI to participate. Hence, these findings may not be truly representative of all the experiences of KTRs in the United States. 
Table 5-2. Possible system implementation of Chronic Care Model concepts and goals for kidney transplant recipient management

\begin{tabular}{|c|c|c|}
\hline CCM Concepts & Goals for KTR Management & System Implementation \\
\hline $\begin{array}{l}\text { Healthcare } \\
\text { Organization }\end{array}$ & $\begin{array}{l}\text { Promote a culture of patient- } \\
\text { and provider-centered quality } \\
\text { care and research in kidney } \\
\text { transplantation }\end{array}$ & $\begin{array}{l}\text { Healthcare supports chronic care } \\
\text { management in long term kidney } \\
\text { transplantation } \\
\text { - Value Based Care Models that } \\
\text { recognize the complexity of } \\
\text { KTR care } \\
\text { - Incentivize integrated CCM } \\
\text { care for all people with kidney } \\
\text { disease. } \\
\text { - Provision of continuing } \\
\text { Medicare coverage including } \\
\text { pharmacy } \\
\text { - Standardized transition plans } \\
\text { between transplant centers } \\
\text { and community nephrologists }\end{array}$ \\
\hline $\begin{array}{l}\text { Community } \\
\text { Resources \& Policy }\end{array}$ & $\begin{array}{l}\text { Assist patient access to needed } \\
\text { services }\end{array}$ & $\begin{array}{l}\text { Proactive programs in place to } \\
\text { access community services } \\
\text { - Nephrology work with } \\
\text { community leaders to develop } \\
\text { needed services } \\
\text { - Transportation } \\
\text { - Job placement } \\
\text { - Support groups } \\
\text { - Exercise programs }\end{array}$ \\
\hline $\begin{array}{l}\text { Delivery Systems } \\
\text { Design }\end{array}$ & $\begin{array}{l}\text { Deliver quality, effective, } \\
\text { coordinated and integrated care }\end{array}$ & $\begin{array}{l}\text { Provider practice supports access } \\
\text { to patient-centered, proactive, } \\
\text { efficient, quality care } \\
\text { - Planned regular visits } \\
\text { including primary and } \\
\text { preventive care visits } \\
\text { - Ensure access that is patient- } \\
\text { centered-including extended } \\
\text { clinic hours and group visits } \\
\text { - Provide multi-disciplinary } \\
\text { care with clear roles and care } \\
\text { plans to include: } \\
\text { Nurse Practitioners } \\
\text { Social Work } \\
\text { Pharmacy } \\
\text { Dieticians } \\
\text { Mental Health }\end{array}$ \\
\hline
\end{tabular}


Table 5-2. (Continued)

\begin{tabular}{|c|c|c|}
\hline CCM Concepts & Goal for KTR Management & System Implementation \\
\hline $\begin{array}{l}\text { Clinical } \\
\text { Information } \\
\text { Systems }\end{array}$ & $\begin{array}{l}\text { Organize patient and } \\
\text { population data to facilitate } \\
\text { quality multidisciplinary care } \\
\text { plans }\end{array}$ & $\begin{array}{l}\text { Provider Electronic Health Records } \\
\text { supports quality care } \\
\text { - Provides patient registries to } \\
\text { ensure provision of evidence } \\
\text { based chronic illness and } \\
\text { preventive care measures } \\
\text { - Provides access to all patient } \\
\text { medical orders \& results } \\
\text { - Supports coordination of care } \\
\text { between other specialty HCPs } \\
\text { - Provides integrated prescription } \\
\text { and pharmacy records to ensure } \\
\text { medication adherence } \\
\text { - Supports regular reporting of } \\
\text { practice quality measures }\end{array}$ \\
\hline Decision Support & $\begin{array}{l}\text { Use evidence-based practice } \\
\text { guidelines in a multi- } \\
\text { disciplinary care team }\end{array}$ & $\begin{array}{l}\text { Ensure evidence-based guidelines } \\
\text { are integrated into care } \\
\text { - Flow sheets } \\
\text { - Utilize chronic disease registry } \\
\text { to alert the multidisciplinary } \\
\text { team to patient specific } \\
\text { laboratory and diagnostic needs, } \\
\text { e.g., HbA1c, microalbuminuria, } \\
\text { ophthalmology visit, foot-checks } \\
\text { \& vaccinations } \\
\text { - Ensure provider continuing } \\
\text { education in transplantation }\end{array}$ \\
\hline $\begin{array}{l}\text { Self-Management } \\
\text { Support }\end{array}$ & $\begin{array}{l}\text { Engage patients \& empower } \\
\text { to manage their health }\end{array}$ & $\begin{array}{l}\text { Ensure patient centered self- } \\
\text { management support needs are } \\
\text { identified \& integrated throughout } \\
\text { multi-disciplinary care plans } \\
\text { - Standardized assessment tools } \\
\text { are utilized to identify specific } \\
\text { patient needs including mental } \\
\text { health. } \\
\text { - Identify patient health goals \& } \\
\text { provide education and } \\
\text { connection with available } \\
\text { community resources to support } \\
\text { those goals. } \\
\text { - Provide ongoing patient } \\
\text { education increasing health } \\
\text { literacy and efficacy }\end{array}$ \\
\hline
\end{tabular}




\section{Conclusions}

At the turn of the 21 st century, the healthcare industry faced mounting reports of producing low-quality services at insurmountable costs. In the seminal report To Err is Human: Building a Safer Health System, Kohn et al. (2009) outlined the systemic problems, reporting thousands of preventable deaths and adverse events due to medical error and poor coordination of care. As new models of U.S. healthcare provision are being tested in an effort to improve quality, documentation of measures that are found in the CCM have become parts of the reimbursement equation.

The increasing burden of kidney disease on society has resulted in long overdue focus on the barriers to innovation in prevention and care of people with this illness. This is particularly true for KTRs. Future models should focus on the clinical endpoints that are relevant to the changes in patient goals and healthcare. As scholarly focus moves from the clinical endpoint of 1-year graft survival, it is incumbent upon kidney transplant providers and the patient community to develop current clinically relevant endpoints. A more robust understanding of care management can be obtained by broadening evaluation to include the following: measures that relate to how health systems are structured, how healthcare is organized, the level of community resources, what policies are in place, how delivery systems are designed, and the capabilities of clinical information systems. In alignment with gaps in measures identified by NQF (2014), assessments also need to be made of the performance of activities that have been demonstrated to contribute to positive health outcomes for patients within delivery systems, decision support, and clinical information systems. Measured outcomes should include those that assess patient and provider perception of the quality of care they have received and their satisfaction with the care experience, including how informed and involved they are in their care and how productive their interactions are with the healthcare team.

Some believe that the current trend toward large mergers in healthcare organizations may result in a shift away from the dialysis-centric focus in nephrology that has prevailed for many decades. For instance, the Aetna-CVS merger with a focus on kidney disease holds promise particularly for CKD preventive measures, however CVS is already exploring entry into the dialysis market as policy makers hope to increase home dialysis (Kuehn, 2018). The payerpharmacy behemoth, with many retail urgent care clinics already operational, could easily focus on hypertension and diabetes management important to nephrology. This type of operation, however, does not readily address the needs of KTRs, many of whom are either reliant on Medicare or Medicare/Medicaid insured. Additionally, mergers such as these would be unlikely to employ providers knowledgeable in the complex care of KTRs. Nephrology could benefit from a refocus on patient care outside dialysis modalities, which has become its "bread and butter." Additionally, as we look for seamless models of care to support people with kidney disease, we must not minimize the importance of the patient and provider relationship. Keeping the importance of the relationship in focus for new models of care and the ensuing reimbursement changes is important to attracting medical students and others members of an integrated care team to nephrology. With approximately 2000 kidney disease patients per nephrologist in the U.S., there is urgency in attracting qualified providers. Currently, life-work balance and low remuneration are key barriers to interest in nephrology among medical students (Nair et al., 2019). 
As policymakers seek methods to provide effective and efficient quality care to people with kidney disease, the often-overlooked KTRs need to be included in these study of care models. In the United States, there are currently 37 organizations with over 500,000 dialysis patients involved in the CMS pilots known as ESCOs. These organizations are similar in structure to Accountable Care Organizations (ACO) and are responsible for providing comprehensive quality care that addresses patients' needs beyond the dialysis center (Gedney \& Kalanter-Zadeh, 2018). The promising early results include an estimated \$75 million in savings and improved outcome measures. Scholars have posited that this coordinated care model will prevail for dialysis care, with CKD and transplant specifically left out of the model.

Exploration into expanding the role of dialysis provider organizations to incorporate long-term management of KTRs, with the nephrologist assuming a more central role in the care model, could reduce the silos of care which exist within the nephrology specialty. A more standardized transition process between transplant centers and the long-term care provider should be considered to ensure that KTRs are not lost to follow-up and providers have access to kidney transplant specific resources. Additionally, practitioners could utilize the existing delivery system design and clinical information systems to create a patient-centered, seamless care model for all people with kidney disease. The effectiveness of the CCM in the long-term care of KTRs could easily be assessed in the dialysis provider community, as most of the CCM elements of care are already integrated in the dialysis care model. The table below provides examples of how the dialysis delivery system infrastructure might adapt to improve quality, patient-centric, seamless care for all people with kidney disease as they navigate multiple social, economic, and healthcare transitions. This proposed model could also reinvigorate the nephrology subspecialty, drawing more interest to the specialty from medical students, nurse practitioners, registered nurses, social workers, pharmacy, and mental health providers. Patients and providers would directly influence shaping the future.

Regardless of the care model employed to provide more integrated, effective, and efficient care, the influence of the patient - provider relationship on patient outcomes and satisfaction deserves a central role. Further, more attention to providing patient specific, ongoing education could facilitate KTRs transition from dialysis to post transplant care. Kidney transplant recipients have often spent years on dialysis thereby losing some locus of control over their personal lives and health. Providing transplant education early and often could result in more engaged patients and improve quality of life.

\section{Recommendations for Future Research}

Future researchers should focus upon the integration of all the elements of the CCM within the patient care plan with particular attention to creating links between clinical care and community and public health organizations. This may lead to a more comprehensive understanding of effective healthcare management for KTRs. Through this recommendation, the researcher highlights the need to further examine the non-clinical factors that may affect the provision of quality healthcare to KTRs. All future research in system implementation of processes to better manage chronic conditions should include measures of change in patient and provider workloads to adequately assess effectiveness. 
Another opportunity for future researchers is to conduct a more expansive qualitative exploration of the barriers and facilitators of quality healthcare management of KTRs by including the perceptions of primary caretakers. The scope of such a qualitative exploration may also be expanded by focusing more on the psychological factors that affect quality healthcare management. Moreover, the experiences of KTRs may be expanded beyond the scope of exploring the barriers and facilitators of effective healthcare management by focusing on their caretakers or their unique challenges during their recuperation.

Future researchers may conduct more sophisticated statistical analyses by incorporating possible mediating variables that affect the quality healthcare management of KTRs. For instance, researchers could examine the mediating effect of the severity of the case, the psychological willingness of patients, and socioeconomic context in order to achieve a more precise understanding of the relationship of these variables. With the inclusion of these possible mediating variables, future researchers can develop a model that clearly identifies the different factors leading to effective healthcare management for KTRs.

There remains ample opportunity for study in kidney transplant education for both recipients and providers. Specifically, focusing on enhancing the EMR systems to provide patient specific education and patient engagement needs through computer enhanced intelligence that meet patient and provider needs.

Lastly, more study is needed on the influence of the patient - provider relationship in complex conditions on patient outcomes, specifically the possible effects of increasing technology use on the relationship and outcomes. This is especially important as CMS pushes for increased use of home dialysis. In doing so, this will allow researchers to determine if a reduction in patient and $\mathrm{HCP}$ interaction has a negative effect on kidney transplant education, access, and outcomes and therefore inform next steps in order to improve patient outcomes. 


\section{LIST OF REFERENCES}

American Society of Nephrology. (2015). Nephrology workforce. Retrieved from https://www.asn-online.org/education/training/workforce/

American Society of Transplantation. (2015). Managing long-term outcomes for kidney transplant patients: An integrated needs assessment. Retrieved from https://www.myast.org/sites/default/files/pdfs/Kidney_Transplant_Report_12.21.2011FI NAL.pdf

Association of American Medical Colleges. (2015). State physician workforce data book. Retrieved from https://www.aamc.org/data/workforce/reports/442830/ statedataandreports.html

Balbale, S. N., Etingen, B., Malhiot, A., Miskevics, S., \& LaVela, S. L. (2016). Perceptions of chronic illness care among veterans with multiple chronic conditions. Military Medicine, 181(5), 439-44.

Berns, J. S., Ellison, D. H., Linas, S. L., \& Rosner, M. H. (2014). Training the next generations' nephrology workforce. Clinical Journal of the American Society of Nephrology, 9(9), 1639-44.

Bidaut-Russell, M., Gabriel, S. E., Scott, C. G., Zinmeister, A. R., Luthra, H. S., \& Yawn, B. (2002). Determinants of patient satisfaction in chronic illness. Arthritis and Rheumatism, 47, 494-500.

Bodenheimer, T., Wagner, E. H., \& Grumbach, K. (2002). Improving primary care for patients with chronic illnesses. Journal of the American Medical Association, 288(14), 1775-79.

Bonomi, A. E., Wagner, E. H., Glasgow, R. E., \& VonKorff, M. (2002). Assessment of chronic illness care (ACIC): A practical tool to measure quality improvement. Health Services Research, 37(3), 791-820.

Brennan, D. M., Mawson, S., \& Brownsell, S. (2009). Telerehabilitation: Enabling the remote delivery of healthcare, rehabilitation, and self-management. Studies in Health Technology and Informatics, 145, 231-48.

Buckley, B. A., McCarthy, D. M., Forth, V. E., Tanabe, P., Schmidt, M. J., Adams, J. G., \& Engel, K. G. (2013). Patient input into the development and enhancement of ED discharge instructions: A focus group study. Journal of Emergency Nursing, 39(6), 553561. doi:10.1016/j.jen.2011.12.018.

Bujang, M. A. (2017). Feasibility of implementing chronic care model in the Malaysian public primary care setting. Medical Journal of Malaysia, 72(2), 107.

Chung, K. C., \& Shauver, M. J. (2009). Measuring quality in healthcare and its implications for pay-for-performance initiatives. Hand Clinics, 25(1), 71-vii. doi:10.1016/j.hcl.2008.09.001.

Carlin, C. S., Christianson, J. B., Keenan, P., \& Finch, M. (2012). Chronic illness and patient satisfaction. Health Services Research, 47(6), 2250-72.

Centers for Disease Control and Prevention. (2009). Chronic disease. Retrieved from https://www.cdc.gov/chronicdisease/index.htm

Centers for Medicare \& Medicaid Services. (2017). Chronic conditions. Retrieved from https://www.cms.gov/research-statistics-data-and-systems/statistics-trends-andreports/chronic-conditions/cc main.html 
Coleman, K., Austin, B. T., Brach, C., \& Wagner, E. H. (2009). Evidence on the chronic care model in the new millennium. Health Affairs, 28(1), 75-85.

Committee on Quality Healthcare in America, Institute of Medicine. (2001). Crossing the quality chasm: A new health system for the 21st century. Washington, DC: National Academy Press.

Cramm, J. M., Strating, M. M., Tsiachristas, A., \& Nieboer, A. P. (2011). Development and validation of a short version of the Assessment of Chronic Illness Care (ACIC) in Dutch disease management programs. Health and Quality of Life Outcomes, 9(1), 49.

Creating High-Quality Results and Outcomes Necessary to Improve Chronic Act of 2017, S.870, 115d.Cong., 1st Sess. (2017).

Creswell, J. W., \& Plano Clark, V. L. (2011). Designing and conducting mixed methods research (2nd ed.). Thousand Oaks, CA: SAGE.

Curry, L. A., Krumholz, H. M., O’Cathain, A., Plano Clark, V. L., Cherlin, E., \& Bradley, E. H. (2013). Mixed methods in biomedical and health services research. Circulation: Cardiovascular Quality and Outcomes, 6(1), 119-23. doi:10.1161/CIRCOUTCOMES.112.967885.

Daker-White, G., Hays, R., McSharry, J., Giles, S., Churighi-Sohi, S., Rhodes, P., \& Sanders, C. (2015). Blame the patient, blame the doctor, or blame the system? A metasynthesis of qualitative studies of patient safety in primary care. PloS ONE, 10(8), e0128329.

Davy, C., Bleasel, J., Liu, H., Tchan, M., Ponniah, S., \& Brown, A. (2015). Factors influencing the implementation of chronic care models: A systematic literature review. BMC Family Practice, 16(1), 102.

Denzin, N. K., \& Lincoln, Y. S. (1994). Handbook of qualitative research. Thousand Oaks, CA: SAGE.

Delwiche, L. D., \& Slaugher, S. J. (2011). The little SAS book: A primer (5th ed.). Cary, NC: SAS Institute.

Donabedian, A. (1988). The quality of care: How can it be assessed? Journal of the American Medical Association, 260(12), 1743-48.

Fernandez, I. (2006). Care of the nephrologic patient: Validity of a distance coordination with primary assistance. Nefrología, 26(3), 97-102.

Flocke, S. A., \& Litaker, D. (2007). Physician practice patterns and variation in the delivery of preventive services. Journal of General Internal Medicine, 22(2), 191-196. doi:10.1007/s11606-006-0042-y.

Fowler, K. (2018). Innovation in transplantation: Accountability, collaboration, and the value of the patient voice. ASN Kidney News. Retrieved from https://www.kidneynews.org/kidney-news/features/innovation-in-transplantationaccountability-collaboration-and-the-value-of-the-patient-voice

Frandsen, B. R., \& Joynt, K. E. (2015). Care fragmentation, quality, and costs among chronically ill patients. American Journal of Managed Care, 21(5), 355-362.

Gedney, N., \& Kalanter-Zadeh, K. (2018). Dialysis patient-centeredness and precision medicine: Focus on incremental home hemodialysis and preserving residual kidney function. Seminal Nephrology, 38(4), 426-32.

Gill, J. S., Wright, A. J., Delmonico, F. L., \& Newell, K. A. (2017). Towards improving the transfer of care of kidney transplant recipients. American Journal of Transplantation, 17(1), 54-59. 
Glasgow, R., Wagner, E. H., Schaefer, J., Mahoney, L., Reid, R., \& Greene, S. (2005). Development and validation of the patient assessment of chronic illness care (PACIC). Medical Care, 43(5), 436-44.

Gordon, E., Fink, J., \& Fischer, M. (2103). Telemedicine: A novel approach to improve coordinated and collaborative care for chronic kidney disease. Nephrology, Dialysis, Transplantation, 28(4), 972-981.

Government Accountability Office. (2016). Kidney disease research funding and priority setting (GAO-17-121). Retrieved from http://www.gao.gov/assets/690/681715.pdf

Green, J. A., \& Boulware, L. E. (2016). Patient education and support during CKD transitions: When the possible becomes probable. Advances in Chronic Kidney Disease, 23(4), 231239.

Headly, K. (2018). Am I my brother's or sister's keeper? Kidney News, 10(5), 5.

Heisler, M., Bouknight, R. R., Hayward, R. A., Smith, D. M., \& Kerr, E. A. (2002). The relative importance of physician communication, participatory decision making, and patient understanding in diabetes self-management. Journal of General Internal Medicine, 17(4), 243-52.

Held, P. J., McCormick, F., Ojo, A., \& Roberts, J. P. (2016). A cost-benefit analysis of government compensation of kidney donors. American Journal of Transplantation, 16(3), 877-885.

Heldal, K., Hartmann, A., Grootendorst, D., de Jager, D., Leivestad, T., Foss, A., \& Midtvedt, K. (2010). Benefit of kidney transplantation beyond 70 years of age. Nephrology Dialysis Transplantation, 25(5), 1680-1687.

Huneycutt, J. (2015). Trends in nephrology. Nephrology News \& Issues, 29(3), 27-29.

Jackson, B., Oates, G., Singh, K., Shikany, J., Fouad, M, Partridge, E., Bae, S. (2017).

Disparities in chronic conditions in the mid-south. Ethnicity and Health, 22(2), 196-208.

Jackson, G. L., Weinberger, M., Hamilton, N. S., \& Edelman, D. (2008). Racial/ethnic and educational-level differences in diabetes care experiences in primary care. Primary Care Diabetes, 2(1), 39-44.

Jolly, S. E., Navaneethan, S. D., Schold, J. D., Arrigain, S., Konig, V., Burrucker, Y. K., \& Nally, J. V. (2015). Development of a chronic kidney disease patient navigator program. BMC Nephrology, 16(1), 69.

Kawamoto, K., Houlihan, C. A., Balas, E. A., \& Lobach, D. F. (2005). Improving clinical practice using clinical decision support systems: A systematic review of trials to identify features critical to success. British Medical Journal, 330(7494), 765.

Khwaja, A., \& El-Nahas, M. (2012). Transplantation in the obese: Separating myth from reality. Nephrology Dialysis Transplantation, 27(10), 3732-3735.

Kidney Disease: Improving Global Outcomes (KDIGO). (2009). Transplant work group KDIGO clinical practice guideline for the care of kidney transplant recipients. American Journal of Transplantation, 9(Suppl 3), S1-155.

Kohn, L. T., Corrigan, J., \& Donaldson, M. S. (2000). To err is human: Building a safer health system. Washington, DC: National Academy Press.

Königshausen, E., \& Sellin, L. (2017). Recent treatment advances and new trials in adult nephrotic syndrome. BioMed Research International, 2017.

Kralewski, J. E., Dowd, B. E., \& Xu, Y. W. (2012). Medical groups can reduce costs by investing in improved quality of care for patients with diabetes. Health Affairs, 31(8), 1830-35. 
Kramer, M. R., Black, N. C., Matthews, S. A., \& James, S. A. (2017). The legacy of slavery and contemporary declines in heart disease mortality in the U.S. South. SSM - Population Health, 3, 609-17.

Ku, E., Johansen, K. L., Portale, A. A., Grimes, B., \& Hsu, C. (2015). State level variations in nephrology workforce and timing and incidence of dialysis in the United States among children and adults: A retrospective cohort study. BMC Nephrology, 16(2). doi:10.1186/1471-2369-16-2.

Kuehn, B. M. (2018). ASN-FDA collaboration takes on rare kidney disease. ASN Kidney News, 10(6). Retrieved from https://www.asn-online.org/publications/kidneynews

Lincoln, Y. S., \& Guba, E. G. (1985). Naturalistic inquiry. Newbury Park, CA: SAGE.

Lorig, K. R., \& Holman, H. (2003). Self-management education: History, definition, outcomes, and mechanisms. Annals of Behavioral Medicine, 26(1), 1-7.

MacColl Center for Health Care Innovation. (n.d.). Measurement tool. Retrieved from http://maccollcenter.org/our-work/measurement-tool

Mahomed, O. H., \& Asmall, S. (2017). Professional nurses' perceptions and experiences with the implementation of an integrated chronic care model at primary healthcare clinics in South Africa. Curationis, 40(1), 1-6.

Marcelli, S., Gatti, C., Rocchi, R., Troiani, S., Di Tuccio, S., Giuli, C.,...Santarelli, A. (2017). Chronic care model and cost reduction in initial health: A new approach for satisfaction and improvement of chronicity. Geriatric Care, 3(3).

McCaughan, J. A., \& Courtney, A. E. (2015). The clinical course of kidney transplant recipients after 20 years of graft function. American Journal of Transplantation, 15(3), 734-740.

McCoy, R., Fan, J., Smith, S., Deming, J., Ziegenfuss, J., Montori, V., \& Shah, N. (2015). Patient experience with care and clinical outcomes in diabetes: A case for more personcentered measurement. European Journal for Person Centered Healthcare, 3(2), 187-195.

Morse, J. M. (2016). Mixed method design: Principles and procedures (Vol. 4). London, England: Routledge.

Narva, A. S., Norton, J. M., \& Boulware, L. E. (2015). Educating patients about CKD: The path to self-management and patient-centered care. Clinical Journal of the American Society of Nephrology, CJN-07680715.

National Quality Forum. (2013). NQF report of 2013 activities to Congress and the Secretary of the Department of Health and Human Services. Washington, DC: Author.

National Quality Forum. (2014). NQF report of 2014 activities to Congress and the Secretary of the Department of Health and Human Services. Washington, DC: Author.

Noel, P. H., Jones, S., \& Parchman, M. L. (2016). Patient experience in an era of primary care transformation: Revisiting the PACIC. European Journal for Person Centered Healthcare, 4(3), 528.

Nugent, K. E., \& Lambert, V. A. (1996). The advanced practice nurse in collaborative practice. Nursing Connections, 9(1), 5-16.

Ong, S. C., \& Gaston, R. S. (2015). Medical management of chronic kidney disease in the renal transplant recipient. Current Opinion in Nephrology and Hypertension, 24(6), 587-593.

Organ Procurement and Transplantation Network and Scientific Registry of Transplant Recipients. (2015). Annual data report. Retrieved from https://www.srtr.org/reportstools/srtroptn-annual-data-report/ 
Prado, M. Roa, L. M., \& Reina-Tosina, J. (2006). Viability study of a personalized and adaptive knowledge-generation telehealthcare system for nephrology. International Journal of Medical Informatics, 75(9), 646-657.

QSR Internationa. (n.d.). NVivo by QSR. Retrieved from https://www.qsrinternational.com/

Rettig, R. A., Norris, K., \& Nissenson, A. R. (2008). Chronic kidney disease in the United State: A public policy imperative. Clinical Journal of the American Society of Nephrology, 3(6), 1902-10.

Rick, J., Rowe, K., Hann, M., Sibbald, B., Reeves, D., Roland, M., \& Bower, P. (2012). Psychometric properties of the patient assessment of chronic illness care measure: Acceptability, reliability and validity in United Kingdom patients with long-term conditions. BMC Health Services Research, 12(1), 293.

Rumpsfeld M., Arild, E., Norum, J., \& Breivik, E. (2005). Telemedicine in haemodialysis: A university department and two remote satellites linked together as one common workplace. Journal of Telemedicine \& Telecare, 11(5), 251-255.

Saldaña, J. (2013). The coding manual for qualitative researchers. Thousand Oaks, CA: SAGE.

Salsburg, E., Quigley, L., Masselink, L., Wu, X., \& Collins, A. (2015). The US nephrology workforce 2015: Developments and trends. Retrieved from https://www.asnonline.org/education/training/workforce/Nephrology_Workforce_Study_Report 2015.pdf

SAS Institute. (n.d.). SAS 9.4. Retrieved from https://www.sas.com/en_us/home.html

Sendall, M., McCosker, L., Crossley, K., \& Bonner, A. (2017). A structured review of chronic care model components supporting transition between healthcare service delivery types for older people with multiple chronic diseases. Health Information Management Journal, 46(2), 58-68.

Sharif, M., Elsayed, M., Stack, A. (2016). The global nephrology workforce: Emerging threats and potential solutions. Clinical Kidney Journal, 9(1), 11-22.

Squitieri, L., Bozic, K. J., \& Pusic, A. L. (2017). The role of patient-reported outcome measures in value-based payment reform. Value in Health: Journal of the International Society for Pharmacoeconomics and Outcomes Research, 20(6), 834-836. doi:10.1016/j.jval.2017.02.003.

Steinberg, E. A., Moss, M., Buchanan, C. L., \& Goebel, J. (2017). Adherence in pediatric kidney transplant recipients: Solutions for the system. Pediatric Nephrology, 1-12.

Suter, E., Oelke, N., Dias da Silva Lima, M., Stiphout, M., Janke, R., \& Witt, R. (2017). Indicators and measurement tools for health systems integration: A knowledge synthesis. International Journal of Integrated Care, 17(6), 4. doi:10.5334/ijic.3931

Teddlie, C., \& Tashakkori, A. (2009). Foundations of mixed methods research: Integrating quantitative and qualitative approaches in the social and behavioral sciences. London, England: SAGE.

Tuot, D. S., Velasquez, A., McCulloch, C. E., Banerjee, T., Zhu, Y., Hsu, C. Y.,...Powe, N. R. (2015). The Kidney Awareness Registry and Education (KARE) study: Protocol of a randomized controlled trial to enhance provider and patient engagement with chronic kidney disease. BMC Nephrology, 16(1), 166.

Tzvetanov, I., D'Amico, G., Walczak, D., Jeon, H., Garcia-Roca, R., Oberholzer, J., \& Benedetti, E. (2014). High rate of unemployment after kidney transplantation: Analysis of the united network for organ sharing database. Transplantation Proceedings, 46(5), 1290-1294. 
United States Department of Health and Human Services. (2019). Remarks to the National Kidney Foundation. Retrieved from https://www.hhs.gov/about/ leadership/secretary/speeches/2019-speeches/remarks-to-the-national-kidneyfoundation.html

United States Renal Data System. (2018). USRDS annual data report: Epidemiology of kidney disease in the United States. Bethesda, MD: National Institutes of Health, National Institute of Diabetes and Digestive and Kidney Diseases.

Varnell, C. D., Rich, K. L., Nichols, M., Dahale, D., Goebel, J. W., Pai, A. L.,...Modi, A. C. (2017). Assessing barriers to adherence in routine clinical care for pediatric kidney transplant patients. Pediatric Transplantation, 21(7).

Vella, J. (2013). Investigational immunosuppressive drugs and approaches in clinical kidney transplantation. Waltham, MA: UpToDate, Inc.

Wagner, E. H. (1998). Chronic disease management: What will it take to improve care for chronic illness? Effective Clinical Practice, 1(1), 2-4.

Wagner, E. H., Davis, C., Schaefer, J., Von Korff, M., \& Austin, B. (2002). A survey of leading chronic disease management programs: Are they consistent with the literature? Journal of Nursing Care Quality, 16(1), 67.

Werzowa, J., Säemann, M., Haidinger, M., Krebs, M., \& Hecking, M. (2015). Antidiabetic therapy in post kidney transplantation diabetes mellitus. Transplantation Reviews, 29(3), 145-153.

Wetmore, J. B., Liu, J., Li, S., Hu, Y., Peng, Y., Gilbertson, D. T., \& Collins, A. J. (2016). The Healthy People 2020 objectives for kidney disease: How far have we come, and where do we need to go? Clinical Journal of the American Society of Nephrology, CJN-04210416.

Zhang, W., \& Creswell, J. (2013). The use of "mixing" procedure of mixed methods in health services research. Medical Care, 51(8), 51-57. doi:10.1097/MLR.0b013e31824642fd.

Zuccaro, L. (2015). Improving chronic kidney disease management using Wagner's model for chronic care. University of Ottawa Journal of Medicine, 5(1), 17-20.

Zwarenstein, M., \& Reeves, S. (2006). Knowledge translation and interprofessional collaboration: Where the rubber of evidence-based care hits the road of teamwork. Journal of Continuing Education in the Health Professions, 26(1), 46-54. 


\section{APPENDIX A. KTR-INFORMED CONSENT}

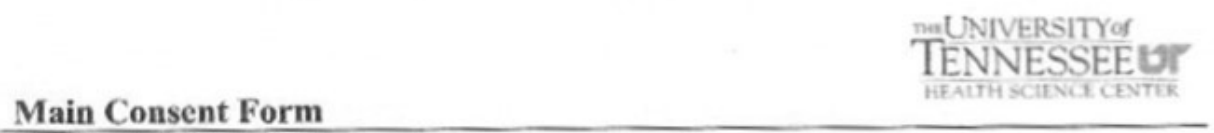

TITLE: Barriers and Facilitators to Adherence of a Post Kidney Transplant Therapeutic Regimen

PRINCIPAL INVESTIGATOR:

FACULTY ADVISOR:
Catherine Pantik, BSN, RN, DNP/PhD Student

920 Madison, Suite 924

Memphis, TN. 38163

Donna Hathaway, PhD, FAAN

\section{INTRODUCTION:}

You are being given the opportunity to participate in this research study. The purpose of this consent form is to help you decide if you want to be in the research study. This consent form may contain words that you do not understand. Please ask the investigator to explain any words or information that you do not clearly understand. Please tell the study doctor or study staff if you are taking part in another research study.

The purpose of this study is to understand what helps a kidney transplant recipient maintain the lifestyle (diet, exercise, lab work, follow-up appointments) and medication changes necessary for the long-term success of the transplanted kidney.

This information will be gathered through kidney transplant recipient participation in focus groups. Focus groups are a type of group interview with the basic purpose being to allow the researcher a more in-depth understanding of the participants' perspectives and experiences.

\section{PROCEDURES TO BE FOLLOWED:}

Thirty-six (36) subjects will be participating in one of three focus groups. Potential participants will be randomly selected from kidney transplant recipients at Methodist University Transplant Institute (MUTI).

The study will take place at MUTI conference room, located at 1211 Union Avenue, Memphis TN 38104.

You will be asked to complete a demographic and bealth status questionnaire. Information such as your age, weight, and medical history will be copied from your medical record at MUTI and/or Methodist University Hospital.

Focus group discussions will be led by the primary researcher, Catherine Pantik, a doctoral student at the University of Tennessee Health Seience Center. The discussions will be centered upon participants' experiences following kidney transplant (i.e., what was helpful or a hindrance to receiving follow-up care, problems with medications, education about caring for your new kidney, etc.), and any other topics that participants would like to discuss.

6-28-15

Page I of 6

Subject Initials

TENY IRB NUMBER: 13-02583-XP

IRB APPROVAL. DATE: 07/10/2015

IRB EXPIRATION DATE: 07/07/2016 
Each focus group will be audio recorded and notes will be taken. At the conclusion of the discussion the moderator will restate and confirm the general findings of the group.

Your participation in this study will last 2 hours on one day.

After each focus group is completed, two participants from each group will be randomly selected to verify the compiled research findings. If randomly selected to verify research findings, you will receive a report in the mail in approximately 3 months. The verification of findings will take approximately 1 hour.

\section{Day 1/Visit 1.}

- Complete demographic and health status questionnaire

- Participate in a focus group discussion with other renal transplant recipients to identify facilitators and barriers to lifestyle and medication changes following transplant

- You might be one of 6 randomly selected participants to review and verify the findings of the rescarch team. The report will be mailed to you with a stamped return envelope.

All activities involved in this study are for research purposes only.

\section{RISKS ASSOCIATED WITH PARTICIPATION:}

There are no inherent risks associated with participation in focus groups. You might experience some psychological effects, such as nervousness or anxiety related to telling others your experiences after renal transplant.

There is the potential risk of loss of confidentiality. Every effort will be made to keep your information confidential; however, this cannot be guaranteed.

Completion of the demographic survey may make you feel uncomfortable or cause troublesome feelings or emotions. You may refuse to answer any of the questions, and you may take a break at any time during the study.

Having your voice recorded may make you feel uncomfortable. You may take a break during any time of the study. There is also a potential risk of loss of confidentiality that someone who listens to your audio recording might identify you. In order to ensure the highest level of confidentiality possible will be maintained, the researchers involved in reviewing the audio tapes are not health care providers at MUTI, and names of participants will not be used during the recorded focus group interviews.

\section{BENEFITS ASSOCIATED WITH PARTICIPATION:}

Although you may not directly benefit from the focus groups, participation may help individuals better understand the common challenges faced by kidney transplant recipients. The results of this study may help people with renal transplants in the future. The information obtained in your 
focus group discussions will be used to develop an intervention that might improve long term outcomes for transplant recipients.

\section{ALTERNATIVES TO PARTICIPATION:}

You will not have to undergo the following procedures if you do not take part in this study:

- Complete a demographic and health status questionnaire

- Have information such as your age, weight, and medical history copied from your medical record

- Review the themes identified from your focus group to verify researcher accuracy in interpretation

You will receive medical treatment for renal transplant follow-up routine care whether or not you participate in the study.

\section{CONFIDENTIALITY:}

Research records

All of your paper research records will be stored in locked file cabinets and will be accessible only to research personnel. All of your electronic research records will be computer password protected and accessible only to research personnel.

Your focus group recordings will be maintained at UTHSC during the study and will be deidentified. A list of the participants' names will not be maintained with the recordings, and no names will be used during the recording of the focus group interviews.

\section{Medical Records}

Information about your participation in this study or the results of procedures performed in this study will be placed in your medical record; as such, this information could be made available to your employer or insurer.

\section{Presentations/Publications}

You will not be identified in any presentations or publications based on the results of this research study.

\section{Authorization to Use and Disclose Information for Research Purposes}

Under federal privacy regulations, you have the right to decide who can review and copy your personal health information (called "protected health information" or PHI). PHI collected in this study may include information such as:

- Past and present medical records

- Records about your study visits

- Records about phone calls made as part of this research

- Research records 
By signing this consent form, you are giving your permission for the study doctor and the study staff at the University of Tennessee to get your PHI from your doctor and/or facilities where you have received health care. They may also share your PHI with:

- The Institutional Review Board (IRB) at the University of Tennessee Health Science Center

- Methodist Healthcare-Memphis Hospitals

Your PHI will only be used and/or given to others:

- To do the rescarch

- To study the results

- To see if the research was done right

You may withdraw or take away your permission to use and disclose your PHI at any time. You do this by sending written notice to the study doctor. If you withdraw your permission, you may not be able to stay in the study.

When you withdraw your permission, no new PHI will be gathered after that date. However, information that has already been gathered may still be used and given to others. The federal regulations allow you to review or copy your PHI that is used in this study.

\section{COMPENSATION AND TREATMENT FOR INJURY:}

You are not waiving any legal rights or releasing the University of Tennessee, Methodist Healthcare-Memphis Hospitals, or the agents of either, from liability for negligence. In the event of physical injury resulting from research procedures, the University of Tennessee and Methodist Healthcare-Memphis Hospitals do not have funds budgeted for compensation for medical treatment. Therefore, the University of Tennessee and Methodist Healthcare-Memphis Hospitals do not provide for treatment or reimbursement for such injuries.

No compensation will be available to you for any non-physical injuries that you may have as a result of research participation, such as legal problems, problems with your finances or job, or damage to your reputation.

\section{QUESTIONS:}

Contact Catherine Pantik at 901-652-0193 if you have questions about your participation in this study, or if you have questions, concerns, or complaints about the research.

You may contact Terrence F. Ackerman, PhD, UTHSC IRB Chairman, at 901-448-4824, or visit the IRB website at

http://www uthsc.cdu/rescarch/research_compliance/IRB/participant complaint.php if you have any questions about your rights as a research subject, or if you have questions, concems, or complaints about the research.

$6-28-15$

Page 4 of 6

Subject Initials

IRB NUMBER: 13-02583-XP
TENVESSE
IRB APPROVAL DATE: 07/10/2015

IRB EXPIRATION DATE: 07/07/2016 


\section{PAYMENT FOR PARTICIPATION:}

You will receive a $\$ 50.00$ (fifty) Visa gift card at the completion of your study visit.

\section{COSTS OF PARTICIPATION:}

There are no costs to you for participating in this study.

\section{VOLUNTARY PARTICIPATION AND WITHDRAWAL:}

Your participation in this research study is voluntary. You may decide not to participate or you may leave the study at any time. Your decision will not result in any penalty or loss of benefits to which you are entitled.

If you are a student of the University of Tennessec, participating or not participating in this study will in no way influence your grade in any course. If you are an employee of the University of Tennessee or Methodist University Hospital, participating or not participating in this study will not affect your employment status.

If you decide to stop being part of the study, you should tell the investigator, and any information that you have already provided will be kept in a confidential manner. 


\section{CONSENT OF SUBJECT:}

You have read or have had read to you a description of the research study as outlined above. The investigator or his/her representative has explained the study to you and has answered all the questions you have at this time. You knowingly and freely choose to participate in the study. A copy of this consent form will be given to you for your records.

Signature of Research Subject (18 years + )

Printed Name of Adult Research Subject
Date

Time

\section{Signature of Person Obtaining Consent}

$\overline{\text { Date }}$ Time

\section{Printed Name of Person Obtaining Consent}

In my judgment, the subject has voluntarily and knowingly given informed consent and possesses the legal capacity to give informed consent to participate in this research study.

Signature of Investigator
Date
Time

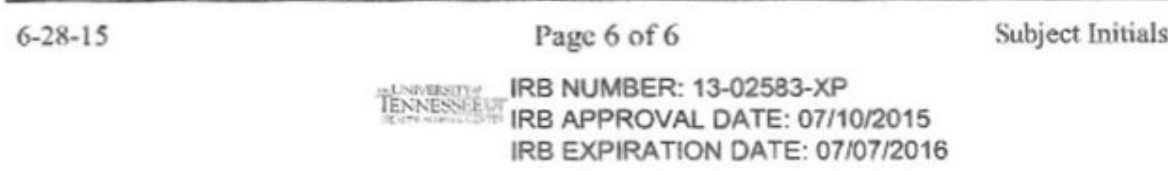




\section{APPENDIX B. KTR DEMOGRAPHIC QUESTIONNAIRE}

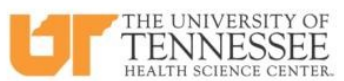

\section{Demographic Questionnaire for Kidney Transplant Recipients}

1. Home Zip Code:

2. Name of Community Nephrologist (Kidney Doctor not at the Transplant Center):

3. Your Age in years: Age at First Transplant:

4. Sex: $\square$ Female $\square$ Male

5. Ethnicity/Race: $\square$ White $\square$ African American $\square$ Other

6. Marital Status: $\square$ Married or with partner $\square$ Not married or without partner

7. Education: $\square<$ High school $\square$ High school graduate or equivalent $\square$ Some college

$\square$ Associates Degree $\square$ College Graduate $\square$ Graduate degree

1

IRB NUMBER: 13-02583-XP

IRB APPROVAL DATE: 08/12/2016 
8. Employment Status: $\square$ Employed $\square$ Unemployed $\square$ Retired $\square$ Disability

9. Gross household income in US\$: $\square<25000 \square 25000-50000 \square 50001-80000 \square$ $>80000$

10. Primary Insurance: $\square$ Private insurance $\square$ Medicare $\square$ Medicaid $\square$ Both Medicare/Medicaid

11. Cause of end-stage renal disease: $\square$ Diabetes $\square$ Hypertension $\square$ Glomenulonephritis $\square$ Polycystic Disease $\square$ Other: _ $\square$ Do not know

12. How many kidney transplants have you had?

13. Do you currently have a functioning transplanted kidney? $\square \mathrm{Y} \quad \square \mathrm{N}$

14. Length of time with current kidney transplant? 
15. Time spent on Dialysis (months, years) or note if transplanted before dialysis. If more than one transplant, please indicate the length of time on dialysis prior to first and other transplants (i.e., Transplant $1=2 \mathrm{yrs}$, Transplant $2=6$ months, Transplant $3=1 \mathrm{yr}$ ):

16. Mode of Dialysis Prior to first transplant:

$\square$ In-Clinic Hemodialysis $\square$ Home Hemodialysis $\square$ Peritoneal

16a. Mode of Dialysis Prior to second transplant (if applicable):

$\square$ In-Clinic Hemodialysis $\square$ Home Hemodialysis $\square$ Peritoneal

16b. Mode of Dialysis Prior to third transplant (if applicable):

$\square$ In-Clinic Hemodialysis $\square$ Home Hemodialysis $\quad \square$ Peritoneal

17. Current Mode of Dialysis if you do not have a functioning graft (kidney) at this time:

$\square$ In-Clinic Hemodialysis $\square$ Home Hemodialysis $\square$ Peritoneal

18. Current Organ Source: $\square$ Deceased Donor $\square$ Living Donor

18a. Organ Source first transplant (if applicable): $\square$ Deceased Donor $\square$ Living Donor

18b. Organ Source second transplant (if applicable): $\square$ Deceased Donor $\square$ Living Donor

19. Number of hospitalizations following your most recent transplant:

20. Length of time (months/years) followed by Methodist Transplant other than annual follow-up visit (if more than one transplant, please note for each, i.e., $1=6$ months, $2=2$ years, $3=9$ months):

21. Did you retum to the care of the nephrologist who referred you for transplant? $\square \mathrm{Y}$ $\mathrm{N}$

21a. If No, please explain: 
22. Smoking History: $\square$ Never a Smoker $\square$ Quit Smoking prior to transplant $\square$ Still a Smoker

23. Alcohol Use: $\square$ Never $\square$ Less than weekly $\square$ 1-4 Drinks per week $\square$ 5-7 Drinks per week

$\square$ More than 7 Drinks per week

24. Physical Activity: $\square$ Not Active: less than 1 hr per week $\square$ Somewhat Active: 1 -3hrs per week

$\square$ Active: 3-5hrs per week $\square$ Very Active: 5hrs per week

Satisfaction with Transplant Experience:

Using the scale below, please indicate your level of satisfaction with the following.

$1=$ Not satisfied $\quad 2$ = Somewhat satisfied $\quad 3$ = Satisfied $\quad 4$ = Very satisfied

\begin{tabular}{|c|c|c|c|c|}
\hline 1. Pre-Transplant "work up" & 1 & 2 & 3 & 4 \\
\hline 2. Post-Transplant Hospital Care & 1 & 2 & 3 & 4 \\
\hline 3. Post-Transplant Care at Transplant Clinic & 1 & 2 & 3 & 4 \\
\hline 4. Care Received by Community Provider Following Transplant & 1 & 2 & 3 & 4 \\
\hline 5. Education about Transplant at Time of "work up"/listing & 1 & 2 & 3 & 4 \\
\hline $\begin{array}{l}\text { 6. Education Received after Transplant: } \begin{aligned} \text { Hospital Care } \\
\text { Transplant Clinic Care } \\
\text { Community Provider }\end{aligned}\end{array}$ & $\begin{array}{l}1 \\
1 \\
1\end{array}$ & $\begin{array}{l}2 \\
2 \\
2\end{array}$ & $\begin{array}{l}3 \\
3 \\
3\end{array}$ & $\begin{array}{l}4 \\
4 \\
4\end{array}$ \\
\hline
\end{tabular}

Please list any other health conditions that require medical care for example high blood pressure, high cholesterol, diabetes, asthma etc......

4

IRB NUMBER: 13-02583-XP

IRB APPROVAL DATE: 08/12/2016 


\title{
APPENDIX C. PACIC SURVEY
}

\section{Assessment of Care for Chronic Conditions}

Staying healthy can be difficult when you have a chronic condition. We would like to learn about the type of help with your condition you get from your health care team. This might include your regular doctor, his or her nurse, or physician's assistant who treats your illness. Your answers will be kept confidential and will not be shared with your physician or clinic.

Over the past 6 months, when I received care for my chronic conditions, I was: made a treatment plan.

A Little of $\underline{\text { Some of }}$ $\underline{\text { the Time }}$

$\underline{\text { the Time }}$

$\underline{\text { Most of }}$

the Time

Always

. Given choices about treatment to think about.

$\square_{1} \quad \square_{2}$

$\square$

$\square_{4}$

$\square_{5}$

Asked to talk about any problems with my medicines or their effects.

$\square_{1}$

$\square_{2}$

$\square_{3}$

$\square_{4}$

$\square_{5}$

$\square_{1} \quad \square_{2}$

$\square_{3}$

$\square_{4}$

$\square_{5}$

4. Given a written list of things I should do to improve my health.

$\square_{1}$

$\square_{2}$

$\square_{3}$

$\square$

$\square_{5}$

5. Satisfied that my care was well organized.

$\square_{1} \quad \square_{2}$

$\square_{2} \quad \square_{3}$

$\square_{3} \quad \square_{4}$

$\square_{5}$

6. Shown how what I did to take care of myself influenced my condition

$\square_{1}$

$\square_{2}$

$\square$

$\square_{4}$

$\square_{5}$

7. Asked to talk about my goals in caring for my condition.

$\square_{1} \quad \square_{2}$

$\square_{2} \quad \square_{3}$

$\square_{4}$

$\square_{5}$

8. Helped to set specific goals to improve my eating or exercise.

9. Given a copy of my treatment plan.

$\square_{1} \quad \square$

$\square_{2} \quad \square_{3}$

$\square_{4}$

$\square_{5}$

$\square$

$\square_{2} \quad \square_{3}$

$\square_{4}$

$\square_{5}$

10. Encouraged to go to a specific group or class to help me cope with my chronic condition.

$\square_{1}$

$\square_{2}$

$\square$

$\square_{4}$

$\square_{5}$

11. Asked questions, either directly or on a survey, about my health habits.

\author{
$\square$
}

$\square$

$\square_{3} \quad \square_{4}$

$\square_{5}$

\section{GroupHealth}

marcoll Conter for Heath Cars Innowation 
Over the past 6 months, when I received care for my chronic conditions, I was:

\begin{tabular}{|c|c|c|c|c|c|}
\hline & $\begin{array}{l}\text { None } \\
\text { of the time }\end{array}$ & $\frac{\text { A Little of }}{\text { the Time }}$ & $\underline{\text { Some of }}$ & $\underline{\underline{\text { Most of }}} \underline{\underline{\text { the Time }}}$ & Always \\
\hline $\begin{array}{l}\text { 12. Sure that my doctor or nurse } \\
\text { thought about my values, beliefs, } \\
\text { and traditions when they } \\
\text { recommended treatments to me. }\end{array}$ & $\square_{1}$ & $\square_{2}$ & $\square_{3}$ & $\square_{4}$ & $\square_{5}$ \\
\hline $\begin{array}{l}\text { 13. Helped to make a treatment plan } \\
\text { that I could carry out in my daily } \\
\text { life. }\end{array}$ & $\square_{1}$ & $\square_{2}$ & $\square_{3}$ & $\square_{4}$ & $\square_{5}$ \\
\hline $\begin{array}{l}\text { 14. Helped to plan ahead so I could } \\
\text { take care of my condition even in } \\
\text { hard times. }\end{array}$ & $\square_{1}$ & $\square_{2}$ & $\square_{3}$ & $\square_{4}$ & $\square_{5}$ \\
\hline $\begin{array}{l}\text { 15. Asked how my chronic condition } \\
\text { affects my life. }\end{array}$ & $\square_{1}$ & $\square_{2}$ & $\square_{3}$ & $\square_{4}$ & $\square_{5}$ \\
\hline $\begin{array}{l}\text { 16. Contacted after a visit to see how } \\
\text { things were going. }\end{array}$ & $\square_{1}$ & $\square_{2}$ & $\square_{3}$ & $\square_{4}$ & $\square_{5}$ \\
\hline $\begin{array}{l}\text { 17. Encouraged to attend programs in } \\
\text { the community that could help } \\
\text { me. }\end{array}$ & $\square_{1}$ & $\square_{2}$ & $\square_{3}$ & $\square_{4}$ & $\square_{5}$ \\
\hline $\begin{array}{l}\text { 18. Referred to a dietitian, health } \\
\text { educator, or counselor. }\end{array}$ & $\square_{1}$ & $\square_{2}$ & $\square_{3}$ & $\square_{4}$ & $\square_{5}$ \\
\hline $\begin{array}{l}\text { 19. Told how my visits with other } \\
\text { types of doctors, like an eye } \\
\text { doctor or other specialist, helped } \\
\text { my treatment. }\end{array}$ & $\square_{1}$ & $\square_{2}$ & $\square_{3}$ & $\square_{4}$ & $\square_{5}$ \\
\hline $\begin{array}{l}\text { 20. Asked how my visits with other } \\
\text { doctors were going. }\end{array}$ & $\square_{1}$ & $\square_{2}$ & $\square_{3}$ & $\square_{4}$ & $\square_{5}$ \\
\hline
\end{tabular}

Copyright 1996-2012 The MacColl Center. The Improving Chronic Illness Care program is supported by The Robert Wood Johnson Foundation, with direction and technical assistance provided by Group Health's MacColl Center for Health Care Innovation

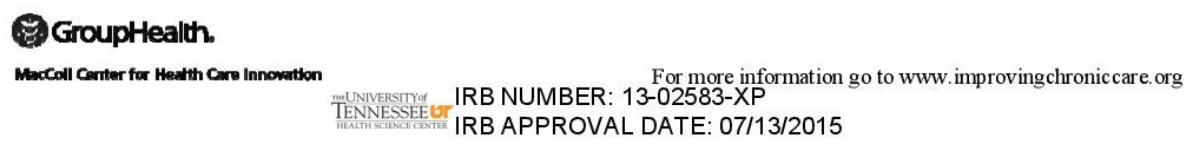

Copyright 1996-2012 The MacColl Center. The Improving Chronic Illness Care program is supported by The Robert Wood Johnson Foundation, with direction and technical assistance provided by Group Health's MacColl Center for Health Care Innovation. Reprinted with permission from Improving Chronic Illness Care. MacColl Center for Health Care Innovation. (n.d.). Measurement tool. Retrieved from http://maccollcenter.org/our-work/measurement-tool 


\section{APPENDIX D. HCP DEMOGRAPHIC QUESTIONNAIRE}

\section{HCP Demographic Survey}

1. Age in years:

2. Gender: $\square$ Male $\square$ Female

3. Ethnicity/Race: $\square$ Caucasian $\square$ African American $\square$ Other

4. Nephrology Board Certification? $\square$ Yes $\square$ No

5. How many years have you been in practice?

6. Individual or Group Practice? (If Group, please note the number of physicians)

7. Number of non-physician employees:

8. Number of days in clinic per week:

9. Clinic Hours: (Please provide days and times):

10. How many KTR's are under your care?

11. Use of Electronic Health Record in clinic? $\square$ Yes $\square$ No

12. Do you use evidence based clinical guidelines (ie KDIGO Guideline for the Care of Kidney Transplant Recipients) in the management of KTR? $\square$ Yes $\square$ No

13. What is the frequency of updates on your kidney transplant care/research? (check only one)
Once a week
Once a month
$\square$ Annually
Rarely

IRB NUMBER: 13-02583-XP

IRB APPROVAL DATE: 08/12/2016 


\section{THE UNIVERSITY OF}

14. What are your preferred references for updates? (check all that apply)
$\square$ Journals
$\square$ Conferences
Books
$\square$ Websites
$\square$ Email Lists

UT TIN IRESER IRB NUMBER: 13-02583-XP

IRB APPROVAL DATE: 08/12/2016 


\section{APPENDIX E. ACIC SURVEY}

\section{Assessment of Chronic Illness Care Version 3.5}

Please complete the following information about you and your organization. This information will not be disclosed to anyone besides the ICIC/IHI team. We would like to get your phone number and e-mail address in the event that we need to contact you/your team in the future. Please also indicate the names of persons (e.g., team members) who complete the survey with you. Later on in the survey, you will be asked to describe the process by which you complete the survey.

\begin{tabular}{|l|l|}
\hline Your name: & Date: \\
\hline Organization \& Address: & Nonth $\frac{1}{\text { Day }}$ \\
\cline { 2 - 3 } & 2. \\
\cline { 2 - 3 } & 3. \\
\hline Your phone of & 2. \\
\hline & Your e-mail address: \\
\hline
\end{tabular}

Directions for Completing the Survey

This survey is designed to help systems and provider practices move toward the "state-of-the-art" in managing chronic illness. The results can be used to help your team identify areas for improvement. Instructions are as follows:

1. Answer each question from the perspective of one physical site (e.g., a practice, clinic, hospital, health plan) that supports care for chronic illness.

Please provide name and type of site (e.g., Group Health Cooperative/Plan)

2. Answer each question regarding how your organization is doing with respect to one disease or condition.

Please specify condition

3. For each row, circle the point value that best describes the level of care that currently exists in the site and condition you chose. The rows in this form present key aspects of chronic illness care. Each aspect is divided into levels showing various stages in improving chronic illness care. The stages are represented by points that range from 0 to 11 . The higher point values indicate that the actions described in that box are more fully implemented.

4. Sum the points in each section (e.g., total part 1 score), calculate the average score (e.g., total part 1 score / \# of questions), and enter these scores in the space provided at the end of each section. Then sum all of the section scores and complete the average score for the program as a whole by dividing this by 6 .

For more information about how to complete the survey, please contact:

Judith Schaefer, MPH

tel. 206.287.2077; Schaefer.jk@ghc.org

Improving Chronic Illness Care

A National Program of the Robert Wood Johnson Foundation

Group Health Cooperative of Puget Sound

1730 Minor Avenue, Suite 1290

Seattle, WA 98101-1448

Copyright 2000 MacColl Institute for Healthcare Innovation, Group Health Cooperative

THUNVERSTYOF IRB NUMBER: 13-02583-XP
TENNESSEE IRT IRB APPROVAL DATE: 07/13/2015 


\section{Assessment of Chronic Illness Care, Version 3.5}

Part 1: Organization of the Healthcare Delivery System. Chronic illness management programs can be more effective if the overall system (organization) in which care is provided is oriented and led in a manner that allows for a focus on chronic illness care.

\begin{tabular}{|c|c|c|c|c|}
\hline Components & Level D & Level C & Level B & Level A \\
\hline $\begin{array}{l}\text { Overall } \\
\text { Organizational } \\
\text { Leadership in Chronic } \\
\text { Illness Care }\end{array}$ & $\begin{array}{l}\text {...does not exist or there is a little } \\
\text { interest. }\end{array}$ & $\begin{array}{l}\text {...is reflected in vision statements } \\
\text { and busincss plans, but no } \\
\text { resources are spccifically } \\
\text { earmarked to execute the work. }\end{array}$ & $\begin{array}{l}\text {...is reflected by senior leadership } \\
\text { and specific dedicated resources } \\
\text { (dollars and personncl). }\end{array}$ & $\begin{array}{l}\text {...is part of the system's long term } \\
\text { planning stratcgy, rcceive } \\
\text { necessary resources, and specific } \\
\text { people are held accountable. }\end{array}$ \\
\hline Score & 1 & $\begin{array}{llll}3 & 4 & 5\end{array}$ & 7 & $\begin{array}{lll}9 & 10 & 11\end{array}$ \\
\hline $\begin{array}{l}\text { Organizational Goals } \\
\text { for Chronic Care }\end{array}$ & $\begin{array}{l}\text {...do not cxist or are limited to one } \\
\text { condition. }\end{array}$ & $\begin{array}{l}\text {...cxist but arc not activcly } \\
\text { reviewed. }\end{array}$ & ...are measurable and revicwed. & $\begin{array}{l}\text {...are measurable, revicwed } \\
\text { routinely, and are incorporated into } \\
\text { plans for improvement. }\end{array}$ \\
\hline Score & 1 & 3 & 7 & $\begin{array}{lll}9 & 10 & 11\end{array}$ \\
\hline $\begin{array}{l}\text { Improvement } \\
\text { Strategy for Chronic } \\
\text { Illness Care }\end{array}$ & $\begin{array}{l}\text {... is ad hoc and not organizcd or } \\
\text { supported consistently. }\end{array}$ & $\begin{array}{l}\text {...utilizes ad hoc approaches for } \\
\text { targeted problems as they emerge. }\end{array}$ & $\begin{array}{l}\text {...utilizes a proven improvement } \\
\text { strategy for targeted problems. }\end{array}$ & $\begin{array}{l}\text {...includes a proven improvement } \\
\text { strategy and uses it proactively in } \\
\text { mecting organizational goals. }\end{array}$ \\
\hline Score & 1 & 4 & 6 & $\begin{array}{llll}9 & 10 & 11\end{array}$ \\
\hline $\begin{array}{l}\text { Incentives and } \\
\text { Regulations for } \\
\text { Chronic IIlness Care }\end{array}$ & $\begin{array}{l}\text {...are not used to influence clinical } \\
\text { performance goals. }\end{array}$ & $\begin{array}{l}\text {... are used to influcnce utilization } \\
\text { and costs of chronic illness care. }\end{array}$ & $\begin{array}{l}\text {...are used to support patient care } \\
\text { goals. }\end{array}$ & $\begin{array}{l}\text {...arc used to motivate and } \\
\text { empower providers to support } \\
\text { patient carc goals. }\end{array}$ \\
\hline & 1 & 4 & 7 & $\begin{array}{lll}9 & 10 & 11 \\
9\end{array}$ \\
\hline Senior Leaders & $\begin{array}{l}\text {... discourage cnrollment of the } \\
\text { chronically ill. }\end{array}$ & $\begin{array}{l}\text {... do not makc improvements to } \\
\text { chronic illness care a priority. }\end{array}$ & $\begin{array}{l}\text {...cncourage improvement cfforts } \\
\text { in chronic care. }\end{array}$ & $\begin{array}{l}\text {..visibly participatc in } \\
\text { improvement efforts in chronic } \\
\text { carc. }\end{array}$ \\
\hline Benefits & $\begin{array}{l}\text {... discourage paticnt sclf- } \\
\text { management or system changes. }\end{array}$ & $\begin{array}{l}\text { ncither cncourage nor } \\
\text { discourage patient self- } \\
\text { management or system changes. }\end{array}$ & $\begin{array}{l}\text {...encourage patient self- } \\
\text { management or system changes. }\end{array}$ & $\begin{array}{l}\text {...are specifically designed to } \\
\text { promote better chronic illness care. }\end{array}$ \\
\hline Score & 1 & 4 & 7 & 11 \\
\hline
\end{tabular}

Total Health Care Organization Score Average Score (Health Care Org. Score / 6) 
Part 2: Community Linkages. Linkages between the health delivery system (or provider practice) and community resources play important roles in the management of chronic illness.

\begin{tabular}{|c|c|c|c|c|}
\hline Components & Level D & Level C & Level B & Level A \\
\hline $\begin{array}{l}\text { Linking Patients to } \\
\text { Outside Resources }\end{array}$ & ...is not done systematically. & $\begin{array}{l}\text {... is limited to a list of identified } \\
\text { community resources in an } \\
\text { accessible format. }\end{array}$ & $\begin{array}{l}\text {...is accomplished through a } \\
\text { designated staff person or resource } \\
\text { responsible for cnsuring providers } \\
\text { and patients make maximum use of } \\
\text { community resources. }\end{array}$ & $\begin{array}{l}\ldots \text { is accomplished through active } \\
\text { coordination between the health } \\
\text { systcm, community scrvicc } \\
\text { agencies and patients. }\end{array}$ \\
\hline Score & 0 & 4 & $\begin{array}{lll}6 & 7 & 8\end{array}$ & 10 \\
\hline $\begin{array}{l}\text { Partnerships with } \\
\text { Community } \\
\text { Organizations }\end{array}$ & ...do not exist. & $\begin{array}{l}\text {.. are being considered but have } \\
\text { not yet boen implemented. }\end{array}$ & $\begin{array}{l}\text {...are formed to develop supportive } \\
\text { programs and policies. }\end{array}$ & $\begin{array}{l}\text {.. are actively sought to develop } \\
\text { formal supportive programs and } \\
\text { policics across the cntirc svstcm }\end{array}$ \\
\hline Score & 0 & 4 & 7 & $\begin{array}{lll}9 & 10 & 11\end{array}$ \\
\hline Regional Health Plans & $\begin{array}{l}\text {...do not coordinate chronic illness } \\
\text { guidclincs, mcasurcs or carc } \\
\text { resources at the practice level. }\end{array}$ & $\begin{array}{l}\text {... would consider some degree of } \\
\text { coordination of guidelincs, } \\
\text { measures or care resources at the } \\
\text { practice level but have not yet } \\
\text { implemented changes. }\end{array}$ & $\begin{array}{l}\text {...currently coordinate guidelines, } \\
\text { mcasurcs or carc rcsources in one } \\
\text { or two chronic illness areas. }\end{array}$ & $\begin{array}{l}\text {...currently coordinate chronic } \\
\text { illncss guidclincs, mcasures and } \\
\text { resources at the practice level for } \\
\text { most chronic illnesses. }\end{array}$ \\
\hline score & 1 & 4 & 8 & 10 \\
\hline
\end{tabular}

Total Community Linkages Score

Average Score (Community Linkages Score / 3 ) 
Part 3: Practice Level. Several components that manifest themselves at the level of the individual provider practice (e.g. individual clinic) have been shown to improve chronic illness care. These characteristics fall into general areas of self-management support, delivery system design issues that directly affect the practice, decision support, and clinical information systems

Part 3a: Self-Management Support. Effective self-management support can help patients and families cope with the challenges of living with and treating chronic illness and reduce complications and symptoms.

\begin{tabular}{|c|c|c|c|c|}
\hline Components & Level D & Level C & Level B & Level A \\
\hline $\begin{array}{l}\text { Assessment and } \\
\text { Documentation of } \\
\text { Self-Management } \\
\text { Needs and Activities }\end{array}$ & ... are not done. & ... are expected. & $\begin{array}{l}\text {...are completed in a standardized } \\
\text { manner. }\end{array}$ & $\begin{array}{l}\text {...are regularly assessed and } \\
\text { rccorded in standardized form } \\
\text { linked to a treatment plan available } \\
\text { to practicc and paticnts. }\end{array}$ \\
\hline Score & 1 & 3 & 7 & $9 \begin{array}{lll}9 & 10 & 11\end{array}$ \\
\hline $\begin{array}{l}\text { Self-Management } \\
\text { Support }\end{array}$ & $\begin{array}{l}\text {.. is limited to the distribution of } \\
\text { information (pamphlets, booklets). }\end{array}$ & $\begin{array}{l}\text {... is available by referral to self- } \\
\text { management classes or educators. }\end{array}$ & $\begin{array}{l}\text {...is provided by trained clinical } \\
\text { educators who are designaled to do } \\
\text { sclf-management support, affiliated } \\
\text { with each practice, and see patients } \\
\text { on referral. }\end{array}$ & $\begin{array}{l}\text {...is provided by clinical educators } \\
\text { affiliated with each practice, } \\
\text { traincd in paticnt cmpowerment } \\
\text { and problem-solving } \\
\text { methodologics, and sec most } \\
\text { patients with chronic illness. }\end{array}$ \\
\hline Score & 1 & 4 & 7 & $9 \quad 10 \quad 11$ \\
\hline $\begin{array}{l}\text { Addressing Concerns } \\
\text { of Patients and } \\
\text { Families }\end{array}$ & ... is not consistently done. & $\begin{array}{l}\text {...is provided for specific patients } \\
\text { and families through referral. }\end{array}$ & $\begin{array}{l}\text {...is encouraged, and peer support, } \\
\text { groups, and mentoring programs } \\
\text { are available. }\end{array}$ & $\begin{array}{l}\text {...is an integral part of care and } \\
\text { includes systematic assessment and } \\
\text { routine involvement in peer } \\
\text { support, groups or mentoring } \\
\text { programs. }\end{array}$ \\
\hline Score & $\begin{array}{ll}0 & 1\end{array}$ & 4 & $\begin{array}{lll}6 & 7 & 8\end{array}$ & $\begin{array}{lll}9 & 10 & 11\end{array}$ \\
\hline $\begin{array}{l}\text { Effective Behavior } \\
\text { Change Interventions } \\
\text { and Peer Support }\end{array}$ & ....are not available. & $\begin{array}{l}\text {... are limited to the distribution of } \\
\text { pamphlets, booklets or other } \\
\text { written information. }\end{array}$ & $\begin{array}{l}\text {...are available only by referral to } \\
\text { specialized centers staffed by } \\
\text { trained personnel. }\end{array}$ & $\begin{array}{l}\text {...are readily available and an } \\
\text { integral part of routine care. }\end{array}$ \\
\hline Score & 1 & $4 \quad 5$ & 8 & 10 \\
\hline
\end{tabular}

Total Self-Management Score

Average Score (Self Management Score / 4) 
Part 3b: Decision Support. Effective chronic illness management programs assure that providers have access to evidence-based information necessary to care for patients--decision support. This includes evidence-based practice guidelines or protocols, specialty consultation, provider education, and activating patients to make provider teams aware of effective therapies.

\begin{tabular}{|c|c|c|c|c|}
\hline Components & Level D & Level C & Level B & Level A \\
\hline $\begin{array}{l}\text { Evidence-Based } \\
\text { Guidelines }\end{array}$ & ....are not available. & $\begin{array}{l}\text {... are available but are not } \\
\text { integrated into care delivery. }\end{array}$ & $\begin{array}{l}\text {...are available and supporled by } \\
\text { provider cducation. }\end{array}$ & $\begin{array}{l}\text {...are available, supporled by } \\
\text { provider cducation and integrated } \\
\text { into carc through reminders and } \\
\text { other proven provider behavior } \\
\text { change methods. }\end{array}$ \\
\hline Score & $\begin{array}{rr}1 & 2 \\
\end{array}$ & 4 & 7 & $\begin{array}{lll}9 & 10 & 11 \\
\end{array}$ \\
\hline $\begin{array}{l}\text { Involvement of } \\
\text { Specialists in } \\
\text { Improving Primary } \\
\text { Care }\end{array}$ & $\begin{array}{l}\text {...is primarily through traditional } \\
\text { referral. }\end{array}$ & $\begin{array}{l}\text {...is achieved through specialist } \\
\text { lcadership to cnhance the capacity } \\
\text { of the overall system to routinely } \\
\text { implement guidelines. }\end{array}$ & $\begin{array}{l}\text {...includes specialist leadership } \\
\text { and designatcd specialists who } \\
\text { provide primary care team training. } \\
6 \quad 7\end{array}$ & $\begin{array}{l}\text {...includes specialist leadership } \\
\text { and specialist involvcment in } \\
\text { improving the care of primary care } \\
\text { patients. }\end{array}$ \\
\hline Score & $\begin{array}{ll}0 & 1\end{array}$ & $\begin{array}{ccc}3 & 4 & 5 \\
\end{array}$ & & $\begin{array}{lll}9 & 10 & 11\end{array}$ \\
\hline $\begin{array}{l}\text { Provider Education } \\
\text { for Chronic Illness } \\
\text { Care }\end{array}$ & ... is provided sporadically. & $\begin{array}{l}\text {. is providcd systematically } \\
\text { through traditional methods. }\end{array}$ & $\begin{array}{l}\text {...is provided using optimal } \\
\text { methods (e.g. academic detailing). }\end{array}$ & $\begin{array}{l}\text {...includes training all practicc } \\
\text { teams in chronic illness care } \\
\text { methods such as population-bascd } \\
\text { management, and self-management } \\
\text { support. }\end{array}$ \\
\hline Score & 1 & 4 & 7 & $\begin{array}{lll}9 & 10 & 11\end{array}$ \\
\hline $\begin{array}{l}\text { Informing Patients } \\
\text { about Guidelines }\end{array}$ & ...is not done. & $\begin{array}{l}\text {...happens on request or through } \\
\text { system publications. }\end{array}$ & $\begin{array}{l}\text {... is done through specific patient } \\
\text { education materials for each } \\
\text { guidclinc. }\end{array}$ & $\begin{array}{l}\text {...includes specific materials } \\
\text { developed for patients which } \\
\text { dcscribc thcir rolc in achicving } \\
\text { guideline adherence. }\end{array}$ \\
\hline Score & 0 & 3 & 8 & $\begin{array}{ccc}9 & 10 & 11\end{array}$ \\
\hline
\end{tabular}

Total Decision Support Score

Average Score (Decision Support Score / 4) 
Part 3c: Delivery System Design. Evidence suggests that effective chronic illness management involves more than simply adding additional interventions to a current system focused on acute care. It may necessitate changes to the organization of practice that impact provision of care.

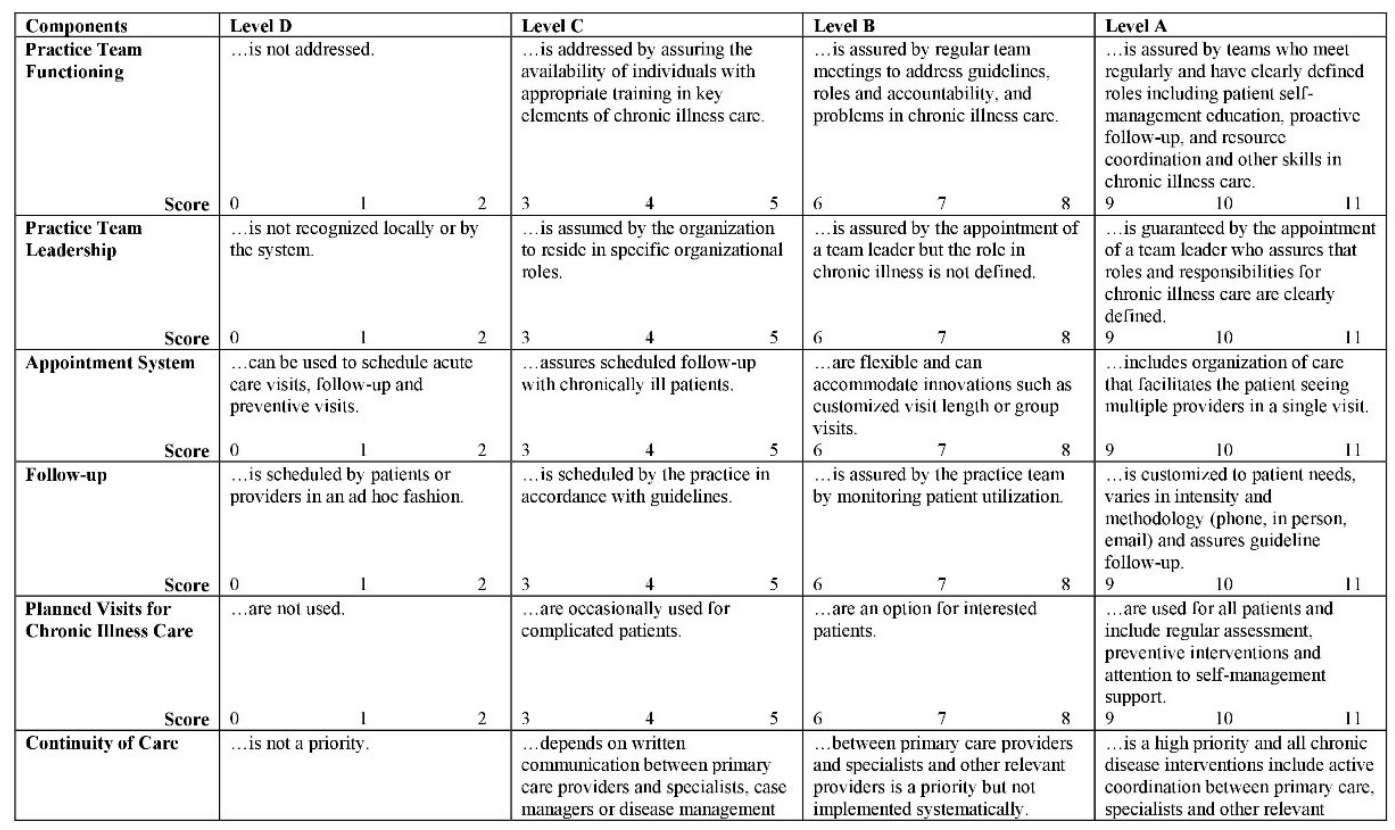

Copyright 2000 MacColl Institute for Healthcare Innovation Group Hcalth Coopcrative
lusversy

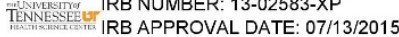




\begin{tabular}{|c|c|c|c|c|c|c|c|c|}
\hline Components & Level D & & Level C & & Level B & & Level A & \\
\hline Score & 1 & 2 & $\begin{array}{l}\text { companics. } \\
3\end{array}$ & 5 & 6 & 8 & $\begin{array}{l}\text { groups. } \\
9\end{array}$ & 11 \\
\hline
\end{tabular}

(From Previous Page)

Total Delivery System Design Score

Average Score (Delivery System Design Score / 6)

Part 3d: Clinical Information Systems. Timely, useful information about individual patients and populations of patients with chronic conditions is a critical feature of effective programs, especially those that employ population-based approaches

\begin{tabular}{|c|c|c|c|c|}
\hline Components & Level D & Level C & Level B & Level A \\
\hline $\begin{array}{l}\text { Registry (list of } \\
\text { patients with specific } \\
\text { conditions) }\end{array}$ & ...is not available. & $\begin{array}{l}\text {...includes name, diagnosis, } \\
\text { contact information and date of last } \\
\text { contact cither on paper or in a } \\
\text { computer database. }\end{array}$ & $\begin{array}{l}\text {...allows qucrics to sort sub- } \\
\text { populations by clinical priorities. }\end{array}$ & $\begin{array}{l}\text {...is ticd to guidelines which } \\
\text { provide prompts and reminders } \\
\text { about needed services. }\end{array}$ \\
\hline Score & 0 & $3 \quad 4 \quad 4 \quad 5$ & 7 & 11 \\
\hline $\begin{array}{l}\text { Reminders to } \\
\text { Providers }\end{array}$ & ...are not available. & $\begin{array}{l}\text {... include general notification of } \\
\text { the cxistence of a chronic illness, } \\
\text { but does not describe needed } \\
\text { services at time of encounter. }\end{array}$ & $\begin{array}{l}\text {...includes indications of needed } \\
\text { scrvicc for populations of patients } \\
\text { through periodic reporting. }\end{array}$ & $\begin{array}{l}\text {...includes specific information for } \\
\text { the team about guideline adherence } \\
\text { at the time of individual patient } \\
\text { encounters. }\end{array}$ \\
\hline Score & 0 & $\begin{array}{lll}3 & 4 & 5\end{array}$ & 7 & $\begin{array}{lll}9 & 10 & 11\end{array}$ \\
\hline Feedback & $\begin{array}{l}\text {... is not available or is non-specific } \\
\text { to the team. }\end{array}$ & $\begin{array}{l}\text {... is provided at infrequent } \\
\text { intervals and is delivered } \\
\text { impersonally. }\end{array}$ & $\begin{array}{l}\text {..occurs at frequent enough } \\
\text { intervals to monitor performance } \\
\text { and is specific to the tcam's } \\
\text { population. } \\
6\end{array}$ & $\begin{array}{l}\text {...is timely, specific to the team, } \\
\text { routine and personally delivered by } \\
\text { a respected opinion leader to } \\
\text { improve team performance. } \\
9\end{array}$ \\
\hline $\begin{array}{l}\text { Information about } \\
\text { Relevant Subgroups } \\
\text { of Patients Needing } \\
\text { Services }\end{array}$ & ....is not available. & $\begin{array}{l}\text {.... an only be obtained with } \\
\text { special efforts or additional } \\
\text { programming. }\end{array}$ & $\begin{array}{l}\text {...can be obtained upon request but } \\
\text { is not routinely available. }\end{array}$ & $\begin{array}{l}\text {... is provided routincly to } \\
\text { providers to help them deliver } \\
\text { planned care. }\end{array}$ \\
\hline Score & 0 & 4 & 8 & 10 \\
\hline $\begin{array}{l}\text { Patient Treatment } \\
\text { Plans }\end{array}$ & ....are not expected. & $\begin{array}{l}\text {...are achieved through a } \\
\text { standardized approach. }\end{array}$ & $\begin{array}{l}\text {... are established collaboratively } \\
\text { and include self management as } \\
\text { well as clinical goals. }\end{array}$ & $\begin{array}{l}\text {... are established collaborative an } \\
\text { include self management as well as } \\
\text { clinical management. Follow-up } \\
\text { occurs and guides care at every } \\
\text { point of service. }\end{array}$ \\
\hline Score & 0 & 4 & 7 & $\begin{array}{lll}9 & 10 & 11 \\
\end{array}$ \\
\hline
\end{tabular}


Total Clinical Information System Score

Average Score (Clinical Information System Score / 5)

Integration of Chronic Care Model Components. Effective systems of care integrate and combine all elements of the Chronic Care Model; e.g., linking patients' self-management goals to information systems/registries.

\begin{tabular}{|c|c|c|c|c|}
\hline Components & Little support & Basic support & Good support & Full support \\
\hline $\begin{array}{l}\text { Informing Patients } \\
\text { about Guidelines }\end{array}$ & ... is not done. & $\begin{array}{l}\text {...happens on request or through } \\
\text { system publications. }\end{array}$ & $\begin{array}{l}\text {...is done through specific patient } \\
\text { education materials for each } \\
\text { guideline. }\end{array}$ & $\begin{array}{l}\text {...includes specific materials } \\
\text { developed for patients which } \\
\text { describe their role in achieving } \\
\text { guideline adherence. }\end{array}$ \\
\hline Score & 1 & $3 \quad 4$ & $\begin{array}{ll}6 & 7 \\
\end{array}$ & $\begin{array}{lll}9 & 10 & 11\end{array}$ \\
\hline $\begin{array}{l}\text { Information } \\
\text { Systems/Registries }\end{array}$ & $\begin{array}{l}\text {...do not include patient self- } \\
\text { management goals. }\end{array}$ & $\begin{array}{l}\text {...include results of patient } \\
\text { assessments (e.g., functional status } \\
\text { rating; readiness to engage in self- } \\
\text { management activities), but no } \\
\text { goals. }\end{array}$ & $\begin{array}{l}\text {...include results of patient } \\
\text { assessments, as well as self- } \\
\text { management goals that are } \\
\text { developed using input from the } \\
\text { practice team/provider and patient. }\end{array}$ & $\begin{array}{l}\text {...include results of patient } \\
\text { assessments, as well as self- } \\
\text { management goals that are } \\
\text { developed using input from the } \\
\text { practice team and patient; and } \\
\text { prompt reminders to the patient } \\
\text { and/or provider about follow-up } \\
\text { and periodic re-evaluation of goals. }\end{array}$ \\
\hline Score & 1 & 4 & 8 & 10 \\
\hline Community Programs & $\begin{array}{l}\text {...do not provide feedback to the } \\
\text { health care system/clinic about } \\
\text { patients' progress in their programs. }\end{array}$ & $\begin{array}{l}\text {...provide sporadic feedback at joint } \\
\text { meetings between the community } \\
\text { and health care system about } \\
\text { patients' progress in their programs. }\end{array}$ & $\begin{array}{l}\text {...provide regular feedback to the } \\
\text { health care system/clinic using } \\
\text { formal mechanisms (e.g., Internet } \\
\text { progress report) about patients' } \\
\text { progress. }\end{array}$ & $\begin{array}{l}\text {...provide regular feedback to the } \\
\text { health care system about patients' } \\
\text { progress that requires input from } \\
\text { patients that is then used to modify } \\
\text { programs to better meet the needs } \\
\text { of patients. }\end{array}$ \\
\hline Score & 1 & 4 & 7 & 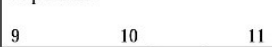 \\
\hline $\begin{array}{l}\text { Organizational } \\
\text { Planning for Chronic } \\
\text { Illness Care }\end{array}$ & $\begin{array}{l}\text {..does not involve a population- } \\
\text { based approach. }\end{array}$ & $\begin{array}{l}\text {... uses data from information } \\
\text { systems to plan care. }\end{array}$ & $\begin{array}{l}\text {... uses data from information } \\
\text { systems to proactively plan } \\
\text { population-based care, including the } \\
\text { development of self-management } \\
\text { programs and partnerships with } \\
\text { community resources. } \\
6\end{array}$ & $\begin{array}{l}\text {...uses systematic data and input } \\
\text { from practice teams to proactively } \\
\text { plan population-based care, } \\
\text { including the development of self- } \\
\text { management programs and } \\
\text { community partnerships, that } \\
\text { include a built-in evaluation plan to } \\
\text { determine success over time. }\end{array}$ \\
\hline
\end{tabular}

Copyright 2000 MacColl Institute for Healthcarc Innovation Group Hcalth Coopcrative

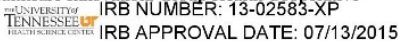




\begin{tabular}{|c|c|c|c|c|c|}
\hline Components & Little support & & Basic support & Good support & \begin{tabular}{|l|} 
Full support \\
\end{tabular} \\
\hline Score & \begin{tabular}{|l|l}
0 & 1 \\
\end{tabular} & 2 & $\begin{array}{ll}3 & 4 \\
\end{array}$ & & \begin{tabular}{|l|l|}
9 & 10 \\
\end{tabular} \\
\hline \multirow[t]{2}{*}{$\begin{array}{l}\text { Routine follow-up for } \\
\text { appointments, patient } \\
\text { assessments and goal } \\
\text { planning }\end{array}$} & \multicolumn{2}{|l|}{...is not ensured. } & $\begin{array}{l}\text { is sporadically done, usually for } \\
\text { appointments only. }\end{array}$ & $\begin{array}{l}\text { is ensured by assigning } \\
\text { responsibilities to specific staff (e.g., } \\
\text { nurse case manager). }\end{array}$ & $\begin{array}{l}\text { is ensured by assigning } \\
\text { responsibilities to specific staff (e.g., } \\
\text { nurse case manager) who uses the } \\
\text { registry and other prompts to } \\
\text { coordinate with patients and the } \\
\text { entire practice team. }\end{array}$ \\
\hline & 1 & 2 & 4 & 7 & 11 \\
\hline \multirow[t]{2}{*}{$\begin{array}{l}\text { Guidelines for chronic } \\
\text { illness care }\end{array}$} & \multicolumn{2}{|l|}{....are not shared with patients. } & $\begin{array}{l}\text {....are given to patients who express } \\
\text { a specific interest in self- } \\
\text { management of their condition. }\end{array}$ & $\begin{array}{l}\text {... are provided for all patients to } \\
\text { help them develop effective self- } \\
\text { management or behavior } \\
\text { modification programs, and identify } \\
\text { when they should see a provider. }\end{array}$ & $\begin{array}{l}\text {...are reviewed by the practice team } \\
\text { with the patient to devise a self- } \\
\text { management or behavior } \\
\text { modification program consistent } \\
\text { with the guidelines that takes into } \\
\text { account patient's goals and readines } \\
\text { to change. }\end{array}$ \\
\hline & 0 & 2 & 4 & 7 & 10 \\
\hline
\end{tabular}

Total Integration Score (SUM items):

$>\quad$ Average Score (Integration Score/6) = 
Briefly describe the process you used to fill out the form (e.g., reached consensus in a face-to-face meeting; filled

out by the team leader in consultation with other team members as needed; each team member filled out a separate form and the responses were averaged).

Description:

Scoring Summary

(bring forward scoring at end of each section to this page)

Total Org. of Health Care System Score

Total Community Linkages Score

Total Self-Management Score

Total Decision Support Score

Total Delivery System Design Score

Total Clinical Information System Score

Total Integration Score

Overall Total Program Score (Sum of all scores)

Average Program Score (Total Program /7) 
What does it mean?

The ACIC is organized such that the highest "score" (an "11") on any individual item, subscale, or the overall score (an average of the six ACIC subscale scores) indicates optimal support for chronic illness. The lowest possible score on any given item or subscale is a "0", which corresponds to limited support for chronic illness care. The interpretation guidelines are as follows:

Between " 0 " and " 2 " = limited support for chronic illness care

Between " 3 " and " 5 " = basic support for chronic illness care

Between "6" and " 8 " = reasonably good support for chronic illness care

Between "9" and "11" = fully developed chronic illness care

It is fairly typical for teams to begin a collaborative with average scores below " 5 " on some (or all) areas the ACIC. After all, if everyone was providing optimal care for chronic illness, there would be no need for a chronic illness collaborative or other quality improvement programs. It is also common for teams to initially believe they are providing better care for chronic illness than they actually are. As you progress in the

Collaborative, you will become more familiar with what an effective system of care involves. You may even notice your ACIC scores "declining" even though you have made improvements; this is most likely the result of your better understanding of what a good system of care looks like. Over time, as your understanding of good care increases and you continue to implement effective practice changes, you should see overall improvement on your ACIC scores

Copyright 2000 MacColl Institute for Healthcarc Innovation Group Hcalth Coopcrative

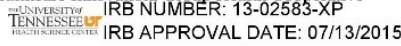

Copyright 1996-2000 The MacColl Center. The Improving Chronic Illness Care program is supported by The Robert Wood Johnson Foundation, with direction and technical assistance provided by Group Health's MacColl Center for Health Care Innovation. Reprinted with permission from Improving Chronic Illness Care. MacColl Center for Health Care Innovation. (n.d.). Measurement tool. Retrieved from http://maccollcenter.org/our-work/measurement-tool 


\title{
APPENDIX F. KTR FOCUS GROUP GUIDE
}

\author{
KTR Focus Group
}

Focus Group Guide

1) Describe the activities that you believe to be necessary for good results with your kidney.

Medications, diet, exercise, glucose testing, follow up medical appointments. Are some of the things / activities easier or harder than others. Discuss what makes some activities more difficult than others. (physical, social, monetary)

2) Talk about the support that you have at home or in the community to perform all the things that are needed for good results with your kidney.

3) Discuss how the Transplant Institute can help you with these activities.

4) Discuss what you do to remember the things you need to do for your health

5) Talk about the changes in your life after your transplant.

6) Talk about the education you received before your transplant and after to prepare you for the changes. 


\section{APPENDIX G. HCP INTERVIEW GUIDE}

\section{Provider Interview Guide}

1) Describe the primary medical problems that you see impacting kidney allograft and patient survival long term.

2) Discuss what you believe to be the key activities that kidney transplant patients must perform in order to achieve optimal post- transplant outcomes. Medications, diet, exercise, glucose testing, follow up medical appointments.

3) Discuss your thoughts on how renal transplant patients should be managed/ or followed up.

4) Discuss what you think the barriers and facilitators to improving long -term outcomes with renal transplant patients might be from a psychosocial aspect.

5) Discuss what you think the barriers and facilitators to improving long -term outcomes with renal transplant patients might be when considering the current healthcare system.

6) Describe what you think are key non- modifiable indicators of long- term graft survival. 


\section{APPENDIX H. HCP COVER LETTER}

You are being given the opportunity to participate in a research study with Methodist University Transplant Institute (MUTI), and The University of Tennessee Health Science Center (UTHSC), which seeks to identify patient and provider perceptions of care management following kidney transplantation. A brief description of the provider portion of the study follows along, with a link to the online survey. The survey will take approximately 20 minutes to complete. If you have any questions, please contact Cathy Pantik atcpantik@uthsc.edu or 901-652-

0193. Upon completion of the survey you will receive a \$25 Visa gift card.

Link to Survey https://uthsc.col.qualtrics.com/SE/?SID=SV_eKyCdqknodbVcmp

TITLE: Practices and Perspectives of Long-Term Care Management Following Kidney Transplantation

PRINCIPAL INVESTIGATOR: Catherine Pantik, RN DNP/PhD Student, Donna Hathaway PhD, FAAN (Advisor) Vinaya Rao, MD (Advisor)

UTHSC IRB NUMBER:13-02583-XP

Thank you for your participation in this study.

(Nurse Practitioners, Physician Assistants or Practice Managers with knowledge of practice patterns can complete this survey)

\section{Summary of Proposal: Practices and Perspectives of Long-Term Care Management Following Kidney Transplantation}

The United States possesses one of the most comprehensive kidney transplant registries in the world [i.e., Scientific Registry of Transplant Recipients (SRTR) and United States Renal Data System (USRDS)]. Despite the availability of such a rich data source, nationally representative data on how long term transplant care is structured and delivered is lacking.

Kidney transplant recipients (KTR) represent a unique subset of the population of persons living with multiple chronic conditions. Although kidney transplantation generally confers better health and quality of life than does dialysis, it is not a cure. The continued presence of chronic kidney disease and other chronic illnesses creates a complex web of demands that require ongoing management for KTR and their HCPs. Consequently, communication, coordination of care, delineation of roles and responsibilities, and effective referral procedures are key issues in the management of transplant patients. Despite the fact that KTR represent a complex patient base with multiple chronic conditions, the usefulness of a comprehensive model of care has not been considered for this cohort in the United States.

The Kidney Disease: Improving Global Outcomes (KDIGO) has proposed guidelines to assist practitioners who care for KTRs. These guidelines are comprehensive and based on the best available evidence. The KDIGO guidelines are less specific, however, on how this care should be delivered at specific transplant centers, and previous efforts to characterize the practice patterns were not found in the literature.

The Chronic Care Model (CCM) has become a widely accepted framework for organizing and delivering patient centered, evidence based care for patients with chronic illness within primary care settings.

The CCM describes six elements that health care organizations need to optimize chronic illness care: Decision support, self-management support, clinical information support, and links to community services. 
The specific aims of this study are:

- Describe current practice patterns and the level of chronic illness management implemented in long-term, follow up care of KTR

- Assess the association between provider and patient perceptions of chronic illness management

- Identify barriers and facilitators to providing chronic illness management to KTR

- Identify barriers and facilitators for KTR adherence to therapeutic regimen

Consent:

You are being given the opportunity to participate in a research study. Methodist University Transplant Institute (MUTI), in conjunction with The University of Tennessee Health Science Center (UTHSC), is conducting a study to identify patient and provider perceptions of care management following kidney transplantation.

This study will identify current care management practices, as well as identify barriers and facilitators to providing care that is consistent with elements of patient centered care as outlined in the Chronic Care Model. Nephrologists providing care to persons who received a kidney transplant at MUTI will complete a survey, Assessment of Chronic Illness Care. Approximately twenty minutes will be needed to complete the survey. Upon completion of the survey each participant will receive a $\$ 25$ Visa Gift Card. This study is funded by the College of Nursing. None of the research staff is employed at MUTI, nor is involved in the direct care of the transplant recipients, mitigating any fears associated with open discussion of post transplant care. Although participants may not directly benefit from the interviews, participation may help individuals better understand the common challenges faced by kidney transplant recipients and aid assessing current practices. The results of this study may help people with renal transplants in the future. The information obtained in your interviews will be used to develop an intervention that might improve long term outcomes for transplant recipients.

Confidentiality will be maintained in that providers' names will not be used. Your participation in this research study is voluntary. 


\section{APPENDIX I. KTR COVER LETTER}

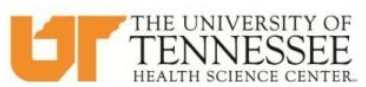

TITLE: Practices and Perspectives of Long-Term Care Management Following Kidney Transplantation

PRINCIPAL INVESTIGATOR: Catherine Pantik, BSN, RN, DNP/PhD Candidate 920 Madison, Suite 924 Memphis, TN. 38163

FACULTY ADVISOR: Donna Hathaway PhD, FAAN

You are being given the opportunity to participate in a research study. Methodist University Transplant Institute (MUTI), in conjunction with the University of Tennessee Health Science Center (UTHSC), is conducting a study to understand the experiences of kidney transplant recipients and long-term care management.

The degree to which long-term care is provided in a manner consistent with patient centered, chronic illness management guidelines will be determined through the responses of kidney transplant recipients to the Patient Assessment of Chronic Illness Care (PACIC) survey and a demographic questionnaire. Approximately twenty minutes will be needed to complete the surveys.

There are no physical risks involved in participation. Although participants may not directly benefit from participation, your participation may help individuals better understand the common challenges faced by kidney transplant recipients and aid in assessing current practices. The information obtained will be used to develop an intervention that might improve long term outcomes for transplant recipients.

Your participation in this research study is voluntary. You may decide not to participate or you may leave the study at any time. Your decision will not result in any penalty.

Confidentiality will be maintained in that kidney transplant recipient's names will not be used. Your responses will be anonymous to the study. Upon receipt of the completed questionnaires, a Visa gift care in the amount of $\$ 15$ will be mailed to you. This study is funded by the College of Nursing.

Again, thank you for your interest in helping us understand the experiences of kidney transplant recipients.

Sincerely,

Cathy Pantik, RN, PhD candidate

University of Tennessee Health Science Center

Memphis, TN 38163

901-652-0193, cpantik@uthsc.edu

$10 / 20 / 2015$

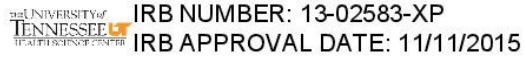




\section{VITA}

Catherine Kelley Pantik was born in Memphis, TN, in 1965. Prior to founding several companies, including two durable medical equipment companies, she received a BA in Psychology and a Certificate in Entrepreneurship from the Cox School of Business from Southern Methodist University and attended Loyola University with coursework in Organizational Psychology. Her diverse background brings a unique perspective to the role and future of nursing.

Ms. Pantik has served as the practice manager and board member of a successful nephrology practice. Her experience working with people with chronic and end-stage renal disease inspired her to pursue her BSN. After graduating Magna Cum Laude from Union University, she worked as a staff nurse in solid organ transplant. Upon enrolling at the University of Tennessee Health Science Center's (UTHSC) College of Nursing $\mathrm{PhD} / \mathrm{DNP}$ program, she has maintained an active role in student leadership. Her continued work in a nephrology clinic contributed to her dissertation research titled Mixed Methods Evaluation of Patient and Provider Perspectives of Chronic Illness Management Following Kidney Transplantation.

As a Jonas Nurse Leader Scholar, she has had the opportunity to present her research at nursing and medical society conferences nationwide. Her published works include committee authorship of the $2^{\text {nd }}$ edition of Scope and Standards of Practice: Transplant Nursing and a secondary data analysis titled Characterization of Body Composition and Fat Mass Distribution One Year After Kidney Transplantation.

She is an active member of the Southern Nursing Research Society (SNRS), is currently the Leadership Council Chair for the American Association of Colleges of Nursing - Graduate Nursing Student Academy (AACN-GNSA), and maintains membership in a number of professional societies including the American Society of Nephrology (ASN) and International Transplant Nurses Society (ITNS).

Dr. Pantik is a certified nurse practitioner who continues to work in the care of people with kidney disease, influencing continued research in social determinants of health, health literacy, chronic illness management, and transitions of care. She is also an Associate Professor at the University of Memphis Loewenberg College of Nursing. Catherine expects to receive the Doctor of Philosophy degree in Nursing Science in December 2019. 